\title{
Modelos de regressão beta com efeitos aleatórios normais e não normais para dados longitudinais
}

\author{
Olga Cecilia Usuga Manco
}

\section{TESE APRESENTADA}

$\mathrm{AO}$

Instituto DE MATEMÁtica e Estatística

DA

Universidade DE SÃo PAUlo

PARA

OBTENÇÃO DO TÍTULO

$\mathrm{DE}$

Doutor EM CIÊNCIAS

\author{
Programa: Estatística \\ Orientadora: Prof. Dra. Viviana Giampaoli
}

Durante o desenvolvimento deste trabalho o autor recebeu auxílio financeiro da $\mathrm{CAPES} / \mathrm{CNPq}$ 


\section{Modelos de regressão beta com efeitos aleatórios normais e não normais para dados longitudinais}

Esta versão da tese contém as correções e alterações sugeridas pela Comissão Julgadora durante a defesa da versão original do trabalho, realizada em 01/03/2013. Uma cópia da versão original está disponível no Instituto de Matemática e Estatística da Universidade de São Paulo.

Comissão Julgadora:

- Profa. Dra. Viviana Giampaoli (orientadora) - IME-USP

- Prof. Dr. Silvia Lopes de Paula Ferrari - IME-USP

- Prof. Dr. Raydonal Ospina Martinez - UFPE

- Prof. Dr. Claudia Regina Oliveira de Paiva Lima - UFPE

- Prof. Dr. Cristian Marcelo Villegas Lobos - ESALQ 


\section{Dedicatoria}

Dedico este trabalho aos meus pais Mariela e Antonio, aos meus irmãos, Liliana e Licinio

e a meu esposo Freddy. 


\section{Agradecimentos}

À minha orientadora, Viviana Giampaoli, pela orientação, apoio, paciência, compreensão e confiança depositada em mim durante a elaboração deste trabalho.

A meu esposo Freddy, pelo amor e carinho, pelo apoio nos momentos difíceis e pelos grandes momentos vividos durante estes quatro anos.

Aos meus pais, Mariela e Antonio, aos meus irmãos Liliana e Licinio e a toda a minha família pelo apoio incondicional.

Aos meus amigos Carmen Elena e Fernando, pela amizade e carinho oferecido durante a estadia em São Paulo.

As minhas amigas e colegas do IME USP, Diana, Nubia, Marina e Elizabeth.

Aos professores Denise Aparecida Botter, Heleno Bolfarine, Silvia Lopes de Paula Ferrari, Gilberto Alvarenga Paula, Carlos Alberto de Bragança Pereira e Chang Chiann, por ter contribuído na minha formação acadêmica e pessoal.

Ao professor Juan Carlos Correa do Departamento de Estadística da Universidad Nacional de Colombia, Sede Medellín, por ter-me incentivado a continuar os estudos de doutorado.

À Coordenação de Aperfeiçoamento de Pessoal de Nível Superior (CAPES) e ao Conselho Nacional de Desenvolvimento Científico e Tecnológico (CNPq) pelo suporte financeiro concedido por meio das bolsas de doutorado.

À Universidade de Antioquia, pela concessão da comissão de estudos, que permitiu a dedicação exclusiva a este trabalho.

Aos professores componentes da minha banca, Viviana Giampaoli, Silvia Lopes de Paula Ferrari, Raydonal Ospina Martinez, Claudia Regina Oliveira de Paiva Lima e Cristian Marcelo Villegas Lobos pelas sugestões e comentários para o melhoramento deste trabalho de tese. 


\section{Resumo}

A classe de modelos de regressão beta tem sido estudada amplamente. Porém, para esta classe de modelos existem poucos trabalhos sobre a inclusão de efeitos aleatórios e a flexibilização da distribuição dos efeitos aleatórios, além de métodos de predição e de diagnóstico no ponto de vista dos efeitos aleatórios. Neste trabalho são propostos modelos de regressão beta com efeitos aleatórios normais e não normais para dados longitudinais. Os métodos de estimação de parâmetros e de predição dos efeitos aleatórios usados no trabalho são o método de máxima verossimilhança e o método do melhor preditor de Bayes empírico. Para aproximar a função de verossimilhança foi utilizada a quadratura de Gauss-Hermite. Métodos de seleção de modelos e análise de resíduos também foram propostos. Foi implementado o pacote BLMM no R para a realização de todos os procedimentos. O processo de estimação dos parâmetros dos modelos e a distribuição empírica dos resíduos propostos foram analisados por meio de estudos de simulação. Foram consideradas várias distribuições para os efeitos aleatórios, valores para o número de indivíduos, número de observações por indivíduo e estruturas de variância-covariância para os efeitos aleatórios. Os resultados dos estudos de simulação mostraram que o processo de estimação obtém melhores resultados quando o número de indivíduos e o número de observações por indivíduo aumenta. Estes estudos também mostraram que o resíduo quantil aleatorizado segue uma distribuição aproximadamente normal. A metodologia apresentada é uma ferramenta completa para analisar dados longitudinais contínuos que estão restritos ao intervalo limitado $(0,1)$.

Palavras-chave: Modelo de regressão beta, máxima verossimilhança, modelos lineares mistos, quadratura de Gauss-Hermite. 


\section{Abstract}

The class of beta regression models has been studied extensively. However, there are few studies on the inclusion of random effects and models with flexible random effects distributions besides prediction and diagnostic methods. In this work we proposed a beta regression models with normal and not normal random effects for longitudinal data. The maximum likelihood method and the empirical Bayes approach are used to obtain the estimates and the best prediction. Also, the Gauss-Hermite quadrature is used to approximate the likelihood function. Model selection methods and residual analysis were also proposed. We implemented a BLMM package in $\mathrm{R}$ to perform all procedures. The estimation procedure and the empirical distribution of residuals were analyzed through simulation studies considering differents random effects distributions, values for the number of individuals, number of observations per individual and covariance structures for the random effects. The results of simulation studies showed that the estimation procedure obtain better results when the number of individuals and the number of observations per individual increase. These studies also showed that the empirical distribution of the quantile randomized residual follows a normal distribution. The methodolgy presented is a tool for analyzing longitudinal data restricted to a interval $(0,1)$.

Keywords: Beta regression model, Gauss-Hermite quadrature, linear mixed models, maximum likelihood. 


\section{Sumário}

Lista de Figuras $\quad$ Xv

Lista de Tabelas $\quad$ xvii

1 Introdução $\quad 1$

1.1 Organização do trabalho . . . . . . . . . . . . . . . . . . 2

2 Conceitos 5

2.1 Dados longitudinais . . . . . . . . . . . . . . . . . 5

2.2 Modelo linear normal de efeitos mistos . . . . . . . . . . . . . . . 7

2.2 .1 Modelo . . . . . . . . . . . . . . . . 7

2.2.2 Abordagem marginal . . . . . . . . . . . . . . . . 7

2.2.3 Abordagem condicional . . . . . . . . . . . . . . . 9

2.3 Quadratura de Gauss-Hermite . . . . . . . . . . . . . . . . . . . 9

2.3.1 Quadratura de Gauss-Hermite unidimensional . . . . . . . . . . . . 10

2.3.2 Quadratura de Gauss-Hermite multidimensional . . . . . . . . . . . . 10

2.4 Quadratura de Gauss-Hermite adaptativa . . . . . . . . . . . . . . . . 11

2.5 Algumas distribuições de probabilidades . . . . . . . . . . . . . . . . 12

2.5.1 Distribuição beta . . . . . . . . . . . . . . . . . . . . . 12

2.5.2 Distribuição log-gama . . . . . . . . . . . . . . . . . . . 12

2.5.3 Distribuição t-Student . . . . . . . . . . . . . . . . . 13

2.5.4 Distribuição exponencial potência . . . . . . . . . . . . . . . 13

2.5.5 Distribuições elípticas . . . . . . . . . . . . . . . . . . . . . . . 13

3 Modelo de regressão beta com intercepto aleatório normal 17

3.1 Modelo proposto . . . . . . . . . . . . . . . 20

3.1 .1 Média . . . . . . . . . . . . . . . . . . . 21

3.1 .2 Variância . . . . . . . . . . . . . . . . 22

3.1.3 Covariância e correlação . . . . . . . . . . . . . . . 23

3.2 Estimação . . . . . . . . . . . . . . . . . . . . . . . . . . . . . 24

3.3 Predição dos efeitos aleatórios . . . . . . . . . . . . . . . . . . . 28

3.4 Estudo de simulação . . . . . . . . . . . . . . . . . . . . . . . . . . . . . . . 29 


\section{Modelo de regressão beta com intercepto aleatório não normal}

4.1 Modelo proposto . . . . . . . . . . . . . . . 36

4.1 .1 Modelo beta log-gama . . . . . . . . . . . . . . . . . . 37

4.1 .2 Modelo beta $t$-Student . . . . . . . . . . . . . . . . . . 37

4.1.3 Modelo beta exponencial potência . . . . . . . . . . . . . . 38

4.2 Estimação . . . . . . . . . . . . . . . . . . . . . . . 38

4.3 Predição dos efeitos aleatórios . . . . . . . . . . . . . . . . . . . 40

4.4 Estudo de simulação . . . . . . . . . . . . . . . . . . . . . . 41

4.4.1 Cenário 1: Modelo beta log-gama . . . . . . . . . . . . . . 41

4.4 .2 Cenário 2: Modelo beta t-Student . . . . . . . . . . . . . . . . . . 44

4.4.3 Cenário 3: Modelo beta exponencial potência . . . . . . . . . . . . . . 44

4.5 Conclusões . . . . . . . . . . . . . . . . . . . . . . . . . 46

5 Modelos de regressão beta com intercepto e inclinação aleatória $\quad 49$

5.1 Modelo proposto . . . . . . . . . . . . . . . . . 50

5.1 .1 Modelo beta normal . . . . . . . . . . . . . . . . 52

5.1 .2 Modelo beta $t$-Student . . . . . . . . . . . . . . . 52

5.1 .3 Modelo beta exponencial potência . . . . . . . . . . . . 53

5.2 Estimação . . . . . . . . . . . . . . . . . . . . . . 53

5.3 Estudo de simulação . . . . . . . . . . . . . . . . . . . 54

5.3 .1 Cenário $1 \ldots \ldots \ldots \ldots \ldots \ldots$

5.3 .2 Cenário $2 \ldots \ldots \ldots \ldots \ldots \ldots \ldots$

5.3 .3 Cenário $3 \ldots \ldots \ldots \ldots \ldots \ldots$

5.4 Conclusões . . . . . . . . . . . . . . . . . . . . . . 56

6 Seleção de modelos e análise de resíduos $\quad 59$

6.1 Seleção de modelos . . . . . . . . . . . . . . . . . . . . . . 60

6.1.1 Seleção da estrutura de regressão de $\mu$ e $\sigma \ldots$. . . . . . . . . . . 61

6.1.2 Seleção de efeitos fixos e aleatórios . . . . . . . . . . . . . . . . 61

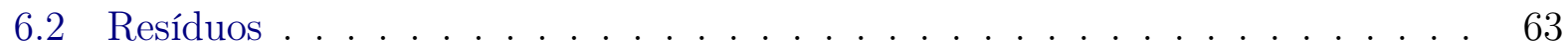

6.2 .1 Resíduo quantil aleatorizado . . . . . . . . . . . . . 63

6.2 .2 Resíduo condicional . . . . . . . . . . . . . . . . . . . . 64

6.2 .3 Resíduo marginal . . . . . . . . . . . . . . . . . . . . 64

6.2 .4 Resíduo aleatório . . . . . . . . . . . . . . . . 64

6.2 .5 Envelope simulado . . . . . . . . . . . . . . . . . . . 64

6.2.6 Distribuição empírica dos resíduos . . . . . . . . . . . . . . . 66

6.3 Conclusões . . . . . . . . . . . . . . . . . . . . . . . 67 
$\begin{array}{lll} & \text { Aplicações } & 77\end{array}$

7.1 Dados de estudo prospectivo oftalmológico . . . . . . . . . . . . . . . . . 77

7.2 Dados de taxa de abandono escolar . . . . . . . . . . . . . . . . . . 82

7.3 Conclusões . . . . . . . . . . . . . . . . . . . 86

8 Pacote BLMM $\quad 89$

9 Conclusões $\quad 105$

9.1 Sugestões para Pesquisas Futuras . . . . . . . . . . . . . . . . . . 106

$\begin{array}{ll}\text { A Função escore e matriz de informação observada } & 109\end{array}$

A.1 Função escore . . . . . . . . . . . . . . . . . . . . . . . . . 109

A.2 Matriz de informação observada . . . . . . . . . . . . . . . . 111

Referências Bibliográficas $\quad 115$ 


\section{Lista de Figuras}

6.1 Gráficos de probabilidade normal dos resíduos quantil aleatorizado, condicional e marginal do modelo beta normal com $\lambda_{1}=0,5 ; N=20 ; n_{i}=3 \mathrm{e}$ $n_{i}=5 \ldots \ldots \ldots \ldots \ldots \ldots \ldots \ldots \ldots \ldots \ldots \ldots \ldots \ldots \ldots \ldots \ldots \ldots$

6.2 Gráficos de probabilidade normal dos resíduos quantil aleatorizado, condicional e marginal do modelo beta normal com $\lambda_{1}=0,5 ; N=20 ; n_{i}=8$ e $n_{i}=12 \ldots \ldots \ldots \ldots \ldots \ldots \ldots \ldots \ldots \ldots \ldots \ldots \ldots \ldots \ldots \ldots$

6.3 Gráficos de probabilidade normal dos resíduos aleatórios para $\mu$ e $\sigma$ do modelo beta normal com $\lambda_{1}=0,5 ; N=20 ; n_{i}=3$ e $n_{i}=5 \ldots \ldots \ldots$

6.4 Gráficos de probabilidade normal dos resíduos aleatórios para $\mu$ e $\sigma$ do modelo beta normal com $\lambda_{1}=0,5 ; N=20 ; n_{i}=8$ e $n_{i}=12$. . . . . . . . . .

6.5 Gráficos de probabilidade normal dos resíduos quantil aleatorizado, condicional e marginal do modelo beta log-gama com $\lambda_{1}=0.22 ; N=20 ; n_{i}=3$ e $n_{i}=5 \ldots \ldots \ldots \ldots \ldots \ldots \ldots \ldots \ldots \ldots \ldots \ldots \ldots \ldots \ldots \ldots$

6.6 Gráficos de probabilidade normal dos resíduos quantil aleatorizado, condicional e marginal do modelo beta log-gama com $\lambda_{1}=0.22 ; N=20 ; n_{i}=8 \mathrm{e}$ $n_{i}=12 \ldots \ldots \ldots \ldots \ldots \ldots \ldots \ldots$

6.7 Gráficos de probabilidade normal dos resíduos aleatórios para $\mu$ e $\sigma$ do modelo beta log-gama com $\lambda_{1}=0.22 ; N=20 ; n_{i}=3$ e $n_{i}=5 \ldots \ldots$. . . . .

6.8 Gráficos de probabilidade normal dos resíduos aleatórios para $\mu$ e $\sigma$ do modelo beta log-gama com $\lambda_{1}=0.22 ; N=20 ; n_{i}=8$ e $n_{i}=12 \ldots \ldots \ldots$

6.9 Gráficos de probabilidade normal dos resíduos quantil aleatorizado, condicional e marginal do modelo beta $t$-Student $\operatorname{com} \nu_{1}=4 ; N=20 ; n_{i}=3 \mathrm{e}$ $n_{i}=5 \ldots \ldots \ldots \ldots \ldots \ldots \ldots \ldots \ldots \ldots \ldots \ldots \ldots \ldots \ldots \ldots \ldots \ldots$

6.10 Gráficos de probabilidade normal dos resíduos quantil aleatorizado, condicional e marginal do modelo beta $t$-Student $\operatorname{com} \nu_{1}=4 ; N=20 ; n_{i}=8 \mathrm{e}$ $n_{i}=12$.

6.11 Gráficos de probabilidade normal dos resíduos aleatórios para $\mu$ e $\sigma$ do modelo beta $t$-Student com $\nu_{1}=4 ; N=20 ; n_{i}=3$ e $n_{i}=5 \ldots \ldots \ldots$

6.12 Gráficos de probabilidade normal dos resíduos aleatórios para $\mu$ e $\sigma$ do modelo beta $t$-Student com $\nu_{1}=4 ; N=20 ; n_{i}=8$ e $n_{i}=12 \ldots \ldots$. . . . 
6.13 Gráficos de probabilidade normal dos resíduos quantil aleatorizado, condicional e marginal do modelo beta exponencial potência com $\lambda_{1}=0.44 ; N=20$;

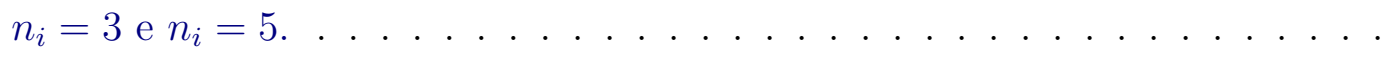

6.14 Gráficos de probabilidade normal dos resíduos quantil aleatorizado, condicional e marginal do modelo beta exponencial potência com $\lambda_{1}=0.44 ; N=20$; $n_{i}=8$ e $n_{i}=12$.

6.15 Gráficos de probabilidade normal dos resíduos aleatórios para $\mu$ e $\sigma$ do modelo beta exponencial potência com $\lambda_{1}=0.44 ; N=20 ; n_{i}=3$ e $n_{i}=5$. . . . .

6.16 Gráficos de probabilidade normal dos resíduos aleatórios para $\mu$ e $\sigma$ do modelo beta exponencial potência com $\lambda_{1}=0.44 ; N=20 ; n_{i}=8$ e $n_{i}=12$. . . .

7.1 Gráfico de perfis da porcentagem observada de volume de gás presente nos olhos dos pacientes segundo os níveis de concentração do gás. . . . . . . . . . 78

7.2 Histograma de frequências e boxplot da pocentagem observada de volume de gás presente nos olhos dos pacientes. . . . . . . . . . . . . . . .

7.3 Gráficos de probabilidade meio-normal com envelope simulado do resíduo quantil aleatorizado, condicional e marginal do modelo beta normal ajustado aos dados da porcentagem de volume de gás presente nos olhos dos pacientes.

7.4 Dados observados e predição da porcentagem de volume de gás presente nos olhos nos pacientes $1,2,17,18,22$ e 31 . . . . . . . . . . . . . 81

7.5 Gráfico de perfis da taxa observada de abandono escolar no ensino fundamental e médio dos municípios segundo a região do estado de Antioquia. . . . . . .

7.6 Histograma de frequências e boxplot da taxa observada de abandono escolar no ensino fundamental e médio nos municípios. . . . . . . . . . . . .

7.7 Dados observados e predição da taxa de abandono escolar no ensino fundamental e médio nos municípios da região L. . . . . . . . . . . . . . . 


\section{Lista de Tabelas}

2.1 Estrutura de dados longitudinais . . . . . . . . . . . . . . . . . . 6

3.1 Funções de ligação. . . . . . . . . . . . . . . . . . . . . . . . . . 21

3.2 REQM das estimativas dos parâmetros do modelo beta normal com $\lambda_{1}=0,5$. 31

3.3 REQM das estimativas dos parâmetros do modelo beta normal com $\lambda_{1}=1,0$. 32

3.4 REQM das estimativas dos parâmetros do modelo beta normal com $\lambda_{1}=1,5$. 32

4.1 REQM das estimativas dos parâmetros do modelo beta log-gama com $\lambda_{1}=0,22.42$

4.2 REQM das estimativas dos parâmetros do modelo beta $\log$-gama com $\lambda_{1}=0,70.43$

4.3 REQM das estimativas dos parâmetros do modelo beta log-gama com $\lambda_{1}=1,22$. 43

4.4 REQM das estimativas dos parâmetros do modelo beta $t$-Student $\operatorname{com} \nu_{1}=4$

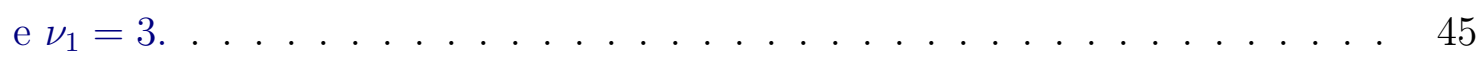

4.5 REQM das estimativas dos parâmetros do modelo beta exponencial potência com $\nu_{1}=\nu_{2}=1,5$ e $\lambda_{1}=0,44 \ldots \ldots \ldots \ldots$. . . . . . . . . 46

4.6 REQM das estimativas dos parâmetros do modelo beta exponencial potência $\operatorname{com} \nu_{1}=\nu_{2}=1,5$ e $\lambda_{1}=0,89 \ldots \ldots \ldots \ldots \ldots \ldots \ldots \ldots \ldots \ldots$

4.7 REQM das estimativas dos parâmetros do modelo beta exponencial potência com $\nu_{1}=\nu_{2}=1,5$ e $\lambda_{1}=1,33 \ldots \ldots \ldots \ldots \ldots$. . . . . . . . 48

5.1 Estruturas de variância-covariância . . . . . . . . . . . . . . . . . 51

5.2 REQM das estimativas dos parâmetros do modelo beta normal considerado no cenário 1. . . . . . . . . . . . . . . . . . . . 57

5.3 REQM das estimativas dos parâmetros do modelo beta normal considerado no cenário 2. . . . . . . . . . . . . . . . . . . . . . . . . . 57

5.4 REQM das estimativas dos parâmetros do modelo beta normal considerado no cenário $3 \ldots \ldots \ldots \ldots \ldots \ldots \ldots$

7.1 Estimativas, erros padrões e valores-p do modelo beta normal ajustado aos dados da porcentagem de volume de gás presente nos olhos. . . . . . . . . . 80

7.2 Modelos beta normal ajustados à estrutura de regressão de $\mu$ e valores AIC. $\quad 84$

7.3 Modelos beta normal ajustados à estrutura de regressão de $\sigma$ e valores AIC. $\quad 84$

7.4 Estimativas, erros padrões e valores-p do modelo beta normal ajustado aos dados de taxa de abandono escolar no ensino fundamental e médio. . . . . . . 


\section{Capítulo 1}

\section{Introdução}

Muitas pesquisas em saúde pública, medicina e ciências sociais são conduzidas por estudos que coletam observações repetidas da variável resposta ao longo do tempo. Esses tipos de pesquisa são referidos como estudos longitudinais e desempenham um papel fundamental na análise de resultados na medida em que seja possível caracterizar possíveis alterações nas características de um indivíduo, associando essas variações com um conjunto de fatores.

Segundo Fitzmaurice et al. (2009) existe uma grande quantidade de desafios na análise de dados longitudinais. Por um lado, devido à sua natureza, as medições repetidas provenientes de estudos longitudinais têm uma estrutura de correlação cuja modelagem desempenha um papel importante na análise desse tipo de dados. Por outro lado, a natureza da variável resposta pode ser contínua ou discreta. Modelos lineares mistos (Laird e Ware (1982)) são a abordagem predominante para a análise de dados longitudinais quando a variável resposta tem distribuição normal e desta forma uma outra modelagem deve ser estudada. No entanto, em muitas aplicações a resposta é limitada pelos valores zero e um. Em outros cenários, segundo Zhang e Davidian (2001), os modelos mistos para dados longitudinais sob suposições de normalidade nos efeitos aleatórios não são suficientemente flexíveis para capturar adequadamente a variação entre indivíduos, assim, o estudo de outros tipos de distribuições são necessários. Este e muitos outros aspectos aumentam a complexidade da análise de alguns conjuntos de dados longitudinais e se faz necessário desenvolver metodologias estatísticas apropriadas que permitam analisar esse tipo de dados medidos en escala contínua.

Com o intuito de abordar a análise de dados longitudinais que assumem valores no intervalo $(a, b)$ desenvolvemos modelos de regressão beta com efeitos aleatórios normais e não normais a fim de considerar a heterogeneidade entre indivíduos num subconjunto de coeficientes de regressão como o intercepto e a inclinação aleatória. Os modelos de regressão beta com interceptos aleatórios normais pertencem à classe de modelos aditivos generalizados para posição, escala e forma (GAMLSS) propostos por Rigby e Stasinopoulos (2005). A classe de modelos de regressão beta com efeitos fixos tem sido estudada amplamente 
por autores como Paolino (2001), Kieschnick e McCullough (2003), Ferrari e Cribari-Neto (2004), Smithson e Verkuilen (2006), Venezuela (2008), Simas et al. (2010), entre outros. Porém, existem poucas publicações, até hoje, sobre a inclusão de efeitos aleatórios na classe de modelos beta e menos ainda considerando uma flexibilização da distribuição dos efeitos aleatórios (Figueroa-Zúñiga et al. (2012)) e nenhum trabalho sobre a predição dos efeitos aleatórios, os métodos de seleção dos modelos e os diagnósticos dos modelos.

É por isso que neste trabalho, além de descrever e desenvolver um método de estimação para os modelos de regressão beta com efeitos aleatórios normais e não normais, realizamos a predição dos efeitos aleatórios, descrevemos um método de seleção dos modelos e propomos resíduos como uma ferramenta de diagnóstico.

\subsection{Organização do trabalho}

A estrutura da tese é como segue. No Capítulo 2 é apresentada uma revisão de conceitos e procedimentos relacionados com a pesquisa deste trabalho.

O modelo de regressão beta com intercepto aleatório normal considerando diferentes funções de ligação é apresentado no Capítulo 3. Assumindo que as respostas dadas os efeitos aleatórios seguem uma distribuição beta derivamos a média, variância, covariância e correlação da distribuição marginal do vetor de respostas observadas. Consideramos para a estimação dos parâmetros o método de máxima verossimilhança. Usamos a quadratura de Gauss-Hermite para aproximar o logaritmo da função de verossimilhança. A partir desta aproximação calculamos as funções escore e a matriz de informação observada associada aos parâmetros do modelo. Também consideramos o problema de predição dos efeitos aleatórios e sugerimos usar a metodologia que considera o melhor preditor de Bayes empírico para obter o preditor dos interceptos aleatórios.

No Capítulo 4 são apresentados os modelos de regressão beta com efeitos aleatórios não normais. Mostramos três modelos particulares com efeitos aleatórios: log-gamma, $t$-Student e exponencial potência. Reformulamos a função de verossimilhança e usamos o método da quadratura de Gauss-Hermite multidimensional para aproximar o logaritmo da função de verossimilhança. Usamos a metodologia que considera o preditor de Bayes empírico para obter o melhor preditor dos interceptos aleatórios. O desempenho do processo de estimação é avaliado numericamente por meio da raiz do erro quadrático médio.

Os modelos de regressão beta com intercepto e inclinação aleatórios e com distribuições elípticas nos efeitos aleatórios são apresentados no Capítulo 5. Além disso, diferentes estruturas de variância-covariância usadas para levar em conta a correlação entre observações de 
um indivíduo ou grupo são consideradas. Estudamos também o efeito destas estruturas nas estimativas dos parâmetros de um modelo de regressão beta com efeitos aleatórios normais bivariados por meio da raiz do erro quadrático médio e da distância relativa entre o vetor de parâmetros e o vetor de estimativas.

No Capítulo 6 propomos um método de seleção de modelos de regressão beta com efeitos aleatórios e duas metodologias para selecionar os efeitos fixos e aleatórios. Também propomos quatro resíduos e estudamos a distribuição empírica deles considerando diferentes distribuições nos interceptos aleatórios.

Duas aplicações com dados reais que ilustram toda a metodologia proposta são apresentadas no Capítulo 7.

No Capítulo 8 descrevemos as funções do pacote BLMM implementado no R para realizar todos os procedimentos de ajuste e seleção dos modelos de regressão beta apresentados. Também descrevemos funções para imprimir o ajuste do modelo, obter o resumo de objetos da classe blmmML e calcular os pontos e pesos da quadratura de Gauss-Hermite.

Finalmente, as conclusões gerais sobre o trabalho aqui desenvolvido são apresentadas no Capítulo 9. 


\section{Capítulo 2}

\section{Conceitos}

Neste capítulo são apresentados os principais tópicos existentes na literatura relacionados ao tema da tese com o intuito de fazer uma revisão que facilite a leitura dos capítulos posteriores. Nas Seções 2.1 e 2.2 realizamos uma breve revisão de dados longitudinais e do modelo linear normal de efeitos mistos. O método da quadratura de Gauss-Hermite usado na estimação dos parâmetros dos modelos propostos é descrito na Seção 2.3. Finalmente, na Seção 2.5 apresentamos as distribuições usadas na formulação dos modelos.

\subsection{Dados longitudinais}

Nas ciências do comportamento, da saúde e sociais, bem como na saúde pública, ciências biológicas e de agricultura, educação e economia os pesquisaddores enfrentam-se com a coleta de dados correlacionados. Segundo Verbeke e Molenberghs (2009) usa-se esse termo em um sentido genérico e entende-se que abrange observações multivariadas, dados agrupados, medidas repetidas, dados longitudinais e dados espacialmente correlacionados. A característica essencial de um estudo com dados longitudinais é que os indivíduos são observados repetidamente ao longo do tempo.

Nos estudos com dados longitudinais $N$ indivíduos são observados em $n_{j}, j=1,2, \ldots, n_{i}$, ocasiões do tempo quanto à variável resposta $y$ e a um conjunto de $p$ variáveis explicativas. O número $n_{i}$ é o número de observações no indivíduo $i$ e $n=\sum_{i=1}^{N} n_{i}$ é o número de observações total no estudo. O vetor de observações de $y$ do indivíduo $i$ é definido como $\boldsymbol{y}_{i}^{\top}=\left(y_{i 1}, y_{i 2}, \ldots, y_{i n_{i}}\right)$, o conjunto completo de $n$ observações é $\boldsymbol{y}^{\top}=\left(\boldsymbol{y}_{1}, \boldsymbol{y}_{2}, \ldots, \boldsymbol{y}_{N}\right)$, a matriz de observações de dimensão $n_{i} \times p$ de $p$ variáveis explicativas do indivíduo $i$ é $\boldsymbol{X}_{i}$ e a matriz de dimensão $n \times p$ que contém as informações das variáveis explicativas de todos os indivíduos é $\boldsymbol{X}$. A Tabela 2.1 apresenta um exemplo de uma estrutura de dados longitudinais. 
Tabela 2.1: Estrutura de dados longitudinais

\begin{tabular}{cccccc}
\hline Indivíduo & Tempo & Variável resposta & \multicolumn{4}{c}{ Variáveis explicativas } \\
\hline 1 & 1 & $y_{11}$ & $x_{111}$ & $\cdots$ & $x_{11 p}$ \\
1 & 2 & $y_{12}$ & $x_{121}$ & $\cdots$ & $x_{12 p}$ \\
$\vdots$ & $\vdots$ & $\vdots$ & $\vdots$ & $\vdots$ & $\vdots$ \\
1 & $n_{1}$ & $y_{1 n_{1}}$ & $x_{1 n_{1} 1}$ & $\cdots$ & $x_{1 n_{1} p}$ \\
\hline 2 & 1 & $y_{21}$ & $x_{211}$ & $\cdots$ & $x_{21 p}$ \\
2 & 2 & $y_{22}$ & $x_{221}$ & $\cdots$ & $x_{22 p}$ \\
$\vdots$ & $\vdots$ & $\vdots$ & $\vdots$ & $\vdots$ & $\vdots$ \\
2 & $n_{2}$ & $y_{2 n_{2}}$ & $x_{2 n_{2} 1}$ & $\cdots$ & $x_{2 n_{2} p}$ \\
\hline$\vdots$ & $\vdots$ & $\vdots$ & $\vdots$ & $\vdots$ & $\vdots$ \\
\hline$N$ & 1 & $y_{N 1}$ & $x_{N 11}$ & $\cdots$ & $x_{N 1 p}$ \\
$N$ & 2 & $y_{N 2}$ & $x_{N 21}$ & $\cdots$ & $x_{N 2 p}$ \\
$\vdots$ & $\vdots$ & $\vdots$ & $\vdots$ & $\vdots$ & $\vdots$ \\
$N$ & $n_{N}$ & $y_{N n_{N}}$ & $x_{N n_{N} 1}$ & $\cdots$ & $x_{N n_{N} p}$ \\
\hline
\end{tabular}

Muitas análises de dados longitudinais baseiam-se num modelo de regressão linear da forma

$$
y_{i j}=\beta_{1} x_{i j 1}+\beta_{2} x_{i j 2}+\ldots+\beta_{p} x_{i j p}+e_{i j}=\boldsymbol{x}_{i j}^{\top} \boldsymbol{\beta}+e_{i j},
$$

em que $i=1,2, \ldots, N, j=1,2, \ldots, n_{i}, \boldsymbol{\beta}=\left(\beta_{1}, \ldots, \beta_{p}\right)^{\top}$ é um vetor $p$-dimensional de coeficientes de regressão desconhecidos e $e_{i j}$ é uma variável aleatória com média zero que representa o desvio da resposta da previsão do modelo, $\boldsymbol{x}_{i j}^{\top} \boldsymbol{\beta}$. Em notação matricial, a equação de regressão para o indivíduo $i$ pode ser escrita como

$$
\boldsymbol{y}_{i}=\boldsymbol{X}_{i} \boldsymbol{\beta}+\boldsymbol{e}_{i}
$$

em que $i=1,2, \ldots, N, \boldsymbol{X}_{i}$ é uma matriz de ordem $\left(n_{i} \times p\right)$ com $\boldsymbol{x}_{i j}$ na linha $j$ e $\boldsymbol{e}_{i}=$ $\left(e_{i 1}, \ldots, e_{i n_{i}}\right)^{\top}$.

\subsection{Modelo linear normal de efeitos mistos}

Nesta seção apresentamos a formulação do modelo linear normal de efeitos mistos para dados longitudinais e as abordagens marginal e condicional associadas.

\subsubsection{Modelo}

Seja $y_{i j}$ o valor da variável resposta do indivíduo $i$ no momento $t_{i j}, i=1, \ldots, N, j=$ $1, \ldots, n_{i}$, e seja $\boldsymbol{y}_{i}=\left(y_{i 1}, y_{i 2}, \ldots, y_{i n_{i}}\right)^{\top}$ o vetor com as $n_{i}$ observações repetidas do indivíduo i. O modelo linear normal de efeitos mistos proposto por Laird e Ware (1982) pode ser escrito 
como

$$
\boldsymbol{y}_{i}=\boldsymbol{X}_{i} \boldsymbol{\beta}+\boldsymbol{Z}_{i} \boldsymbol{b}_{i}+\boldsymbol{e}_{i}, \quad i=1,2, \ldots, N
$$

em que $\boldsymbol{\beta}=\left(\beta_{1}, \beta_{2}, \ldots, \beta_{p}\right)^{\top}$ é o vetor de efeitos fixos, de ordem $p \times 1, \boldsymbol{X}_{i}$ é uma matriz de delineamento conhecida de ordem $n_{i} \times p$ contendo as variáveis explicativas do indivíduo $i$, $\boldsymbol{Z}_{i}$ é uma matriz de delineamento conhecida de ordem $n_{i} \times q, \boldsymbol{b}_{i}=\left(b_{i 1}, b_{i 2}, \ldots, b_{i q}\right)^{\top}$ é o vetor de efeitos aleatórios de, ordem $q \times 1$, e $\boldsymbol{e}_{i}$ é um vetor de erros aleatórios com distribuição normal com média zero e matriz de variância-covariância $\boldsymbol{R}_{i}$ de ordem $n_{i} \times n_{i}$.

Existem duas abordagens para realizar inferências sobre o modelo (2.1): a marginal e a condicional. A primeira é apropriada quando estamos interessados nos coeficientes específicos do indivíduo e os $\boldsymbol{b}_{i}$ são considerados como uma amostra aleatória independente de alguma distribuição. A segunda é adequada quando estamos interessados em um subconjunto de coeficientes de regressão que não varia entre os indivíduos e os $\boldsymbol{b}_{i}$ são tratados como efeitos perturbadores. A seguir apresentamos estas duas metodologias.

\subsubsection{Abordagem marginal}

$\mathrm{Na}$ abordagem marginal o modelo linear normal de efeitos mistos é um modelo que satisfaz

$$
\begin{aligned}
\boldsymbol{y}_{i} & =\boldsymbol{X}_{i} \boldsymbol{\beta}+\boldsymbol{Z}_{i} \boldsymbol{b}_{i}+\boldsymbol{e}_{i}, \\
\boldsymbol{b}_{i} & \sim \boldsymbol{N}_{q}(\mathbf{0}, \boldsymbol{D}), \\
\boldsymbol{e}_{i} & \sim \boldsymbol{N}_{n_{i}}\left(\mathbf{0}, \boldsymbol{R}_{i}\right), \quad i=1,2, \ldots, N
\end{aligned}
$$

em que $\boldsymbol{b}_{1}, \ldots, \boldsymbol{b}_{N}, \boldsymbol{e}_{1}, \ldots, \boldsymbol{e}_{N}$ são mutuamente independentes, $\boldsymbol{D}$ é uma matriz de variânciacovariância de ordem $q \times q$ (definida positiva) e $\boldsymbol{R}_{i}$ é uma matriz de variância-covariância que depende de $i$ apenas por meio da sua dimensão $n_{i}$. As variâncias dos efeitos aleatórios $\boldsymbol{b}_{i}$ medem a variabilidade das trajetórias longitudinais inter-indivíduos; por outro lado, as variâncias dos $\boldsymbol{e}_{i}$ medem a variabilidade das observações repetidas intra-indivíduos.

De (2.2) segue que, condicional aos efeitos aleatórios $\boldsymbol{b}_{i}, \boldsymbol{y}_{i}$ segue uma distribuição normal com vetor de médias $\boldsymbol{X}_{i} \boldsymbol{\beta}+\boldsymbol{Z}_{i} \boldsymbol{b}_{i}$ e com matriz de variância-covariâncias $\boldsymbol{\Sigma}_{i}=\boldsymbol{Z}_{i} \boldsymbol{D} \boldsymbol{Z}_{i}^{T}$. As funções densidade de probabilidade de $\boldsymbol{y}_{i} \mid \boldsymbol{b}_{i}$ e $\boldsymbol{b}_{i}$ são denotados por $f\left(\boldsymbol{y}_{i} \mid \boldsymbol{b}_{i}\right)$ e $f\left(\boldsymbol{b}_{i}\right)$, respectivamente, e a função densidade marginal de $\boldsymbol{y}_{i}$, denotada por $f\left(\boldsymbol{y}_{i}\right)$, é dada por

$$
f\left(\boldsymbol{y}_{i}\right)=\int_{\mathbb{R}^{q}} f\left(\boldsymbol{y}_{i} \mid \boldsymbol{b}_{i}\right) f\left(\boldsymbol{b}_{i}\right) d \boldsymbol{b}_{i} .
$$

Assim $f\left(\boldsymbol{y}_{i}\right)$ é a função densidade de probabilidade de uma distribuição normal de dimensão $n_{i}$ dada por

$$
\boldsymbol{y}_{i} \sim N\left(\boldsymbol{X}_{i} \boldsymbol{\beta}, \boldsymbol{Z}_{i} \boldsymbol{D} \boldsymbol{Z}_{i}^{T}+\boldsymbol{R}_{i}\right)
$$


A média marginal de $\boldsymbol{y}_{i}, \mathrm{E}\left(\boldsymbol{y}_{i}\right)=\boldsymbol{X}_{i} \boldsymbol{\beta}$, pode ser interpretada como uma média sobre todos os efeitos aleatórios, de modo que não corresponde a trajetórias longitudinais individuais. Em vez disso, a inferência individual é realizada pelo condicionamento sobre os efeitos aleatórios $\boldsymbol{b}_{i}$.

A estrutura de variância-covariância das observações repetidas do indivíduo $i$ é dada por

$$
\operatorname{Cov}\left(\boldsymbol{y}_{i}\right)=\boldsymbol{V}_{i}=\boldsymbol{Z}_{i} \boldsymbol{D} \boldsymbol{Z}_{i}^{T}+\boldsymbol{R}_{i}
$$

Essa estrutura indica que a correlação entre as observações repetidas pode ser induzida por meio do termo de variabilidade entre indivíduos, $\boldsymbol{Z}_{i} \boldsymbol{D} \boldsymbol{Z}_{i}^{T}$, ou por meio da matriz de variância-covariância das observações dentro do indivíduo, $\boldsymbol{R}_{i}$. Assim, mesmo se os erros $\boldsymbol{e}_{i}$ são independentes, as observações repetidas $\boldsymbol{y}_{i}$ podem ser correlacionadas devido à variabilidade entre indivíduos.

Diferentes estruturas para $\boldsymbol{D}$ e $\boldsymbol{R}_{i}$ podem ser encontradas na literatura; ver, por exemplo, Wolfinger (1993), Littell et al. (2000), Pinheiro e Bates (2000), Fitzmaurice et al. (2004), Rocha (2004) e Weiss (2005). Uma forma mais simples do modelo marginal surge quando $\boldsymbol{R}_{i}=\sigma^{2} \boldsymbol{I}$, em que $\boldsymbol{I}$ denota a matriz identidade. Nesse caso, o modelo é denominado de modelo de independência condicional uma vez que implica que as $n_{i}$ respostas do indivíduo $i$ são independentes, condicional em $\boldsymbol{b}_{i}$ (Laird e Ware (1982)). Nesse modelo a função densidade de probabilidade dos dados $\boldsymbol{y}_{i}$ dados os efeitos aleatórios $\boldsymbol{b}_{i}$ é dada por

$$
f\left(\boldsymbol{y}_{i} \mid \boldsymbol{b}_{i} ; \boldsymbol{\beta}, \boldsymbol{R}_{i}\right)=\frac{1}{(2 \pi)^{\frac{n_{i}}{2}}\left|\boldsymbol{R}_{i}\right|^{\frac{n_{i}}{2}}} \exp \left\{-\frac{1}{2}\left(\boldsymbol{y}_{i}-\boldsymbol{X}_{i} \boldsymbol{\beta}-\boldsymbol{Z}_{i} \boldsymbol{b}_{i}\right)^{T} \boldsymbol{R}_{i}^{-1}\left(\boldsymbol{y}_{i}-\boldsymbol{X}_{i} \boldsymbol{\beta}-\boldsymbol{Z}_{i} \boldsymbol{b}_{i}\right)\right\} .
$$

A inferência estatística para o modelo linear normal de efeitos mistos sob a abordagem marginal é baseada no método de máxima verossimilhança ou método de máxima verossimilhança restrita. Para maiores detalhes sobre esses dois métodos de estimação ver, por exemplo, Laird e Ware (1982) e Lindstrom e Bates (1988).

\subsubsection{Abordagem condicional}

A abordagem condicional consiste na estimação dos parâmetros desconhecidos por meio da maximização da função de verossimilhança dos dados $\boldsymbol{y}_{i}$ condicional às estatísticas suficientes dos parâmetros perturbadores $\boldsymbol{b}_{i}$.

Segundo Verbeke et al. (2001) e Molenberghs e Verbeke (2005) a principal vantagem da inferência condicional é que não são necessárias suposições adicionais em relação aos parâmetros de perturbação $\boldsymbol{b}_{i}$. Porém, uma desvantagem é que precisam ser encontradas as estatísticas suficientes para os $\boldsymbol{b}_{i}$ e, posteriormente, ser calculada e maximizada a verossimi- 
lhança dos dados condicional a estas estatísticas. Além disso, toda a informação sobre $\boldsymbol{b}_{i}$ é perdida, uma vez que estes parâmetros específicos dos indivíduos indicam a forma como os indivíduos evoluem de forma diferente no tempo.

Para detalhes desta abordagem pode-se consultar Verbeke e Molenberghs (2009).

\subsection{Quadratura de Gauss-Hermite}

Quando se tem interesse em obter a integral de uma função regular $f(z): \mathbb{R} \rightarrow \mathbb{R}$ os métodos de integração numérica baseiam-se na expressão de $f(z)$ como o produto de uma função de peso $w(z): \mathbb{R} \rightarrow \mathbb{R}^{+}$e outra função $g(z): \mathbb{R} \rightarrow \mathbb{R}$, ou seja,

$$
f(z)=w(z) g(z)
$$

Assim, muitos desses métodos aproximam uma integral por uma soma ponderada do tipo

$$
\int_{\mathbb{R}} f(z) d z \approx \sum_{k=1}^{Q} w_{k} g\left(z_{k}\right)
$$

em que os pontos $z_{k}$ denotam os nós e os $w_{k}$ denotam os pesos, e juntos constituem uma regra de integração (Fahrmeir e Tutz, 2001, Apêndice A.4).

\subsubsection{Quadratura de Gauss-Hermite unidimensional}

O método da quadratura de Gauss-Hermite unidimensional aproxima integrais da forma

$$
\int_{-\infty}^{+\infty} \exp \left(-z^{2}\right) g(z) d z
$$

por uma soma ponderada, isto é

$$
\int_{-\infty}^{+\infty} \exp \left(-z^{2}\right) g(z) d z \approx \sum_{k=1}^{Q} w_{k} g\left(z_{k}\right)
$$

em que $g(z), z \in \mathbb{R}$, denota uma função regular, o nó $z_{k}$ é o $k$-ésimo zero do polinômio de Hermite de ordem $Q, H_{Q}(z)$,

$$
H_{Q}(z)=(-1)^{Q} e^{z^{2} / 2} \frac{d^{Q} e^{-z^{2} / 2}}{d z^{Q}}
$$


e os pesos $w_{k}$ dependem do número de nós $Q$ e do polinômio de Hermite $H_{Q-1}(z)$ avaliado em $z_{k}$,

$$
w_{k}\left(z_{k}\right)=\frac{2^{Q-1} Q ! \sqrt{\pi}}{Q^{2}\left(H_{Q-1}\left(z_{k}\right)\right)^{2}} .
$$

\subsubsection{Quadratura de Gauss-Hermite multidimensional}

No método da quadratura de Gauss-Hermite multidimensional considera-se o vetor $m$ dimensional $\boldsymbol{z}=\left(z_{1}, \ldots, z_{m}\right)$, uma função regular $f(\boldsymbol{z}): \mathbb{R}^{m} \rightarrow \mathbb{R}$, uma função de peso $w(\boldsymbol{z}): \mathbb{R}^{m} \rightarrow \mathbb{R}^{+}$e outra função $g(\boldsymbol{z}): \mathbb{R}^{m} \rightarrow \mathbb{R}$, tal que $f(\boldsymbol{z})=w(\boldsymbol{z}) g(\boldsymbol{z})$. A expressão (2.3) pode ser usada para aproximar a integral $m$-dimensional

$$
\int_{\mathbb{R}^{m}} f(\boldsymbol{z}) d \boldsymbol{z}=\int_{\mathbb{R}} \cdots \int_{\mathbb{R}} w\left(z_{1}, \ldots, z_{m}\right) g\left(z_{1}, \ldots, z_{m}\right) d z_{1} \cdots d z_{m}
$$

em que a função de peso é dada por

$$
w(\boldsymbol{z})=\exp \left(-\boldsymbol{z}^{T} \boldsymbol{z}\right)=\exp \left(-z_{1}^{2}\right) \cdot \ldots \cdot \exp \left(-z_{m}^{2}\right)
$$

A seguir a quadratura de Gauss-Hermite unidimensional da forma (2.3) é aplicada a cada um dos componentes de $\boldsymbol{z}$. Usando $Q_{r}$ nós na $r$-ésima dimensão, $r=1,2, \ldots, m$, obtemos a aproximação

$$
\int_{\mathbb{R}^{m}} f(\boldsymbol{z}) d \boldsymbol{z} \approx \sum_{k_{1}=1}^{Q_{1}} w_{k_{1}}^{(1)} \ldots \sum_{k_{m}=1}^{Q_{m}} w_{k_{m}}^{(m)} g\left(z_{k_{1}}^{(1)}, \ldots, z_{k_{m}}^{(m)}\right)
$$

em que $z_{k_{r}}^{(r)}$ é o $k_{r}$-ésimo zero do polinômio de Hermite de ordem $Q_{r}$ e $w_{k_{r}}^{(r)}$ é o peso correspondente. Neste trabalho iremos denotar os $z_{k}$ como os pontos de quadratura e $Q$ como o número de pontos da quadratura. Para maiores detalhes sobre a quadratura de Gauss-Hermite, ver, por exemplo, Evans e Swartz (2000, Capítulo 5) e Fahrmeir e Tutz (2001, Apêndice A.4).

\subsection{Quadratura de Gauss-Hermite adaptativa}

Uma extensão da quadratura de Gauss-Hermite unidimensional é a versão adaptativa (Liu e Pierce, 1994; Pinheiro e Bates, 1995) na qual o objetivo é centrar e espalhar os pontos originais de quadratura ao redor do ponto de máximo $\hat{z}$ da função $\log (f(z))$, isto com o intuito de melhorar a aproximação da integral. A transformação dos pontos de quadratura $z_{k}$ nos novos pontos $z_{k}^{\star}$ é dada pela expressão $z_{k}^{\star}=\sqrt{2} \hat{\sigma} z_{k}+\hat{z}$ em que

$$
\hat{\sigma}^{2}=\left[-\left.\frac{d^{2}}{d z^{2}} \log (f(z))\right|_{z=\hat{z}}\right]^{-1} \text {. }
$$


Assim a aproximação da integral da função $f(z)$ na reta real pela quadratura Gauss-Hermite adaptativa fica dada por

$$
\int_{\mathbb{R}} f(z) d z \approx \sqrt{2} \hat{\sigma} \sum_{k=1}^{Q} f\left(z_{k}^{\star}\right) \exp \left(z_{k}^{2}\right) w_{k}
$$

Em Hernández et al. (2013) é proposto um método para melhorar esta quadratura eliminando combinações de pontos de quadratura que contribuem pouco à aproximação das integrais (pruning).

\subsection{Algumas distribuições de probabilidades}

Nesta seção apresentamos as distribuições que serão utilizadas posteriormente na formulação dos modelos propostos.

\subsubsection{Distribuição beta}

A seguir apresentamos duas parametrizações diferentes da distribuição beta, a primeira em termos da média e da precisão e a segunda em termos da média e da dispersão.

1. A função densidade de probabilidade da distribuição beta com média $\mu$ e precisão $\phi$, denotada por $B e(\mu, \phi)$, é dada por

$$
f(y ; \mu, \phi)=\frac{\Gamma(\phi)}{\Gamma(\mu \phi) \Gamma((1-\mu) \phi)} y^{\mu \phi-1}(1-y)^{(1-\mu) \phi-1},
$$

em que $0<y<1,0<\mu<1$ e $\phi>0$. A média e a variância de $y$ são: $\mathrm{E}(y)=\mu$ e $\operatorname{Var}(y)=\mu(1-\mu) /(1+\phi)$. Mais detalhes sobre esta parametrização podem ser encontrados em Ferrari e Cribari-Neto (2004).

2. A função densidade de probabilidade da distribuição beta com média $\mu$ e dispersão $\sigma$, denotada por $B e(\mu, \sigma)$, é definida como

$$
\begin{aligned}
f(y ; \mu, \sigma)= & \frac{\Gamma\left(\left(1-\sigma^{2}\right) / \sigma^{2}\right)}{\Gamma\left(\mu\left(\left(1-\sigma^{2}\right) / \sigma^{2}\right)\right) \Gamma\left((1-\mu)\left(\left(1-\sigma^{2}\right) / \sigma^{2}\right)\right)} y^{\mu\left(\left(1-\sigma^{2}\right) / \sigma^{2}\right)-1} \\
& \times(1-y)^{(1-\mu)\left(\left(1-\sigma^{2}\right) / \sigma^{2}\right)-1}
\end{aligned}
$$

com $0<y<1,0<\mu<1$ e $0<\sigma<1$. A média e a variância de $y$ são dadas respectivamente por: $\mathrm{E}(y)=\mu \mathrm{e} \operatorname{Var}(y)=\sigma^{2} \mu(1-\mu)$. Mais detalhes sobre esta parametrização podem ser encontrados em Cribari-Neto e Souza (2012). 


\subsubsection{Distribuição log-gama}

A função densidade de probabilidade da distribuição log-gama com parâmetro $\lambda$, denotada por $\mathrm{LG}(\lambda)$, é dada por

$$
f(y ; \lambda)=\frac{\left(\frac{1}{\lambda}\right)^{\frac{1}{\lambda}}}{\Gamma\left(\frac{1}{\lambda}\right)} \exp \left(\frac{y-\exp (y)}{\lambda}\right)
$$

$\operatorname{com} y \in \mathbb{R}$ e $\lambda>0$.

\subsubsection{Distribuição $t$-Student}

A função densidade de probabilidade da distribuição $t$-Student com $\nu$ graus de liberdade, denotada como $t_{\nu}$, é dada por

$$
f(y ; \nu)=\frac{\Gamma\left(\frac{\nu+1}{2}\right)}{\sqrt{\nu \pi} \Gamma\left(\frac{\nu}{2}\right)}\left(1+\frac{y^{2}}{\nu}\right)^{-\frac{(\nu+1)}{2}},
$$

em que $y \in \mathbb{R}$ e $\nu>0$. Note-se que se $\nu=1$ a distribuição $t$-Student é igual a distribuição Cauchy e se $\nu \rightarrow \infty$, a distribuição $t$-Student converge para a distribuição normal padrão.

\subsubsection{Distribuição exponencial potência}

A função densidade de probabilidade da distribuição exponencial potência com parâmetro de locação $\mu$, parâmetro de escala $\lambda$ e parâmetro de curtose $\nu$, denotada como $E P(\mu, \lambda, \nu)$, é dada por

$$
f(y ; \mu, \lambda, \nu)=\frac{1}{2 \nu^{\frac{1}{\nu}} \Gamma\left(1+\frac{1}{\nu}\right) \lambda} \exp \left(-\frac{\left(|y-\mu|^{\nu}\right)}{\nu \lambda^{\nu}}\right),
$$

em que $\mu \in \mathbb{R}, \lambda>0$ e $\nu>0$. Mais detalhes sobre esta parametrização da distribuição exponencial potência podem ser encontrados em Capobianco (2000). Esta distribuição inclui a distribuição de Laplace quando $\nu=1$, a distribuição normal quando $\nu=2$ e a distribuição uniforme quando $\nu \rightarrow \infty$.

\subsubsection{Distribuições elípticas}

A classe de distribuições elípticas é uma classe geral de distribuições com caudas mais leves e mais pesadas do que a normal, como a $t$-Student e a exponencial potência. Suas características e propriedades podem ser encontradas em Fang et al. (1990).

Dizemos que um vetor aleatório $\boldsymbol{Y} \in \mathbb{R}^{p}(p \geq 2)$ segue uma distribuição de contornos elípticos se sua função característica tem a forma

$$
\psi_{\boldsymbol{Y}}(t)=\exp \left\{i t^{\top} \boldsymbol{\mu}\right\} \phi\left(t^{\top} \boldsymbol{\Sigma} t\right),
$$


em que $\boldsymbol{\mu} \in \mathbb{R}^{p}$ denota o parâmetro de posição, $\boldsymbol{\Sigma} \in \mathbb{R}^{p \times p}$ denota o parâmetro de escala (matriz simétrica e positiva semidefinida) e $\phi: \mathbb{R}^{p} \rightarrow \mathbb{R}$ denota o gerador característico da distribuição elíptica, $i=\sqrt{-1}$ e $t \in \mathbb{R}^{p}$.

Se $\boldsymbol{Y}$ tem distribuição elíptica com função característica dada por (2.12), denotamos $\boldsymbol{Y} \sim E l_{p}(\boldsymbol{\mu}, \boldsymbol{\Sigma}, \phi)$ ou simplesmente $\boldsymbol{Y} \sim E l_{p}(\boldsymbol{\mu}, \boldsymbol{\Sigma})$.

Assumindo que o $\operatorname{posto}(\boldsymbol{\Sigma})=p$, temos que a função de densidade do vetor aleatório $\boldsymbol{Y}$ tem a forma

$$
f(y)=|\Sigma|^{-\frac{1}{2}} g\{u\}, \quad y \in \mathbb{R}^{p},
$$

em que $u=(\boldsymbol{y}-\boldsymbol{\mu})^{\top} \boldsymbol{\Sigma}^{-1}(\boldsymbol{y}-\boldsymbol{\mu})$ para alguma função $g,(g(u) \geq 0, u \geq 0)$. A função $g$ é típicamente conhecida como função geradora de densidade, que satisfaz a condição

$$
\int_{0}^{\infty} u^{\frac{p}{2}-1} g(u) d u<\infty
$$

Alguns exemplos de distribuições elípticas são dados a seguir assumindo que posto $(\boldsymbol{\Sigma})=$ $p$.

1. A distribuição normal $p$-variada, denotada por $N_{p}(\boldsymbol{\mu}, \boldsymbol{\Sigma})$ é dada por:

$$
f(y)=(2 \pi)^{-\frac{p}{2}}|\boldsymbol{\Sigma}|^{-\frac{1}{2}} \exp \left\{-\frac{1}{2}(\boldsymbol{y}-\boldsymbol{\mu})^{\top} \boldsymbol{\Sigma}^{-1}(\boldsymbol{y}-\boldsymbol{\mu})\right\},
$$

com

$$
g(u)=(2 \pi)^{-\frac{p}{2}} e^{-\frac{u}{2}}, \quad u \geq 0
$$

2. A distribuição $t$-variada com $\nu$ graus de liberdade, denotada por $t_{p}(\boldsymbol{\mu}, \boldsymbol{\Sigma}, \nu)$, é dada por:

$$
f(y)=\frac{\Gamma\left[\frac{1}{2}(\nu+p)\right]}{\Gamma\left[\frac{\nu}{2}\right](\nu \pi)^{\frac{p}{2}}}|\boldsymbol{\Sigma}|^{-\frac{1}{2}}\left\{1+\frac{1}{\nu}(\boldsymbol{y}-\boldsymbol{\mu})^{\top} \boldsymbol{\Sigma}^{-1}(\boldsymbol{y}-\boldsymbol{\mu})\right\}^{-\frac{1}{2}(\nu+p)}
$$

$\operatorname{com} \nu>0$. Quando $\nu=1$ obtemos a distribuição de Cauchy $p$-variada. Neste caso

$$
g(u)=\frac{\Gamma\left[\frac{1}{2}(\nu+p)\right]}{\Gamma\left[\frac{\nu}{2}\right](\nu \pi)^{\frac{p}{2}}}\left\{1+\frac{u}{\nu}\right\}^{-\frac{1}{2}(\nu+p)}, \quad u \geq 0 .
$$

3. A distribuição exponencial potência $p$-variada com parâmetro de forma $-1 \leq \nu \leq 1$, 
denotada por $E P_{p}(\boldsymbol{\mu}, \boldsymbol{\Sigma}, \nu)$ tem a forma:

$$
f(y)=\frac{p \Gamma\left[\frac{p}{2}\right]}{\pi^{\frac{p}{2}} \Gamma\left[1+\frac{p(1+\nu)}{2}\right] 2^{1+\frac{p(1+\nu)}{2}}}|\boldsymbol{\Sigma}|^{-\frac{1}{2}} \exp \left\{-\frac{1}{2}\left[(\boldsymbol{y}-\boldsymbol{\mu})^{\top} \boldsymbol{\Sigma}^{-1}(\boldsymbol{y}-\boldsymbol{\mu})\right]^{\frac{1}{1+\nu}}\right\},
$$

com

$$
g(u)=\frac{p \Gamma\left[\frac{p}{2}\right]}{\pi^{\frac{p}{2}} \Gamma\left[1+\frac{p(1+\nu)}{2}\right] 2^{1+\frac{p(1+\nu)}{2}}} e^{\frac{1}{2} u^{\frac{1}{1+\nu}}}, \quad u \geq 0 .
$$

Esta distribuição apresenta dois tipos de caudas, mais leves $(\nu<0)$ assim como também mais pesadas do que a normal $(\nu>0)$ e inclui a normal como um caso particular quando $\nu=0$.

Em Fang et al. (1990) pode-se encontrar a demostração da seguinte propriedade que é útil para os cálculos da média e da covariância de distribuições elípticas.

Propriedade Assuma que $\boldsymbol{Y} \sim \operatorname{El}_{p}(\boldsymbol{\mu}, \boldsymbol{\Sigma}, \phi)$ com posto $(\boldsymbol{\Sigma})=r \leq p$. Se o vetor de médias e a matriz de covariância do vetor aleatório $\boldsymbol{Y}$ existem, então $\mathrm{E}(\boldsymbol{Y})=\boldsymbol{\mu} e$ $\operatorname{Cov}(\boldsymbol{Y})=c_{o} \boldsymbol{\Sigma}$, em que $c_{0}=-2 \phi^{\prime}(0)=-2 d \phi(u) /\left.d u\right|_{u=0}$, com $\phi(u)$ sendo o gerador característico da distribuição elíptica.

A seguir são apresentados alguns exemplos desta propriedade.

1. Se $\boldsymbol{Y} \sim N_{p}(\boldsymbol{\mu}, \boldsymbol{\Sigma}), c_{0}=1, \mathrm{E}(\boldsymbol{Y})=\boldsymbol{\mu}$ e $\operatorname{Cov}(\boldsymbol{Y})=\boldsymbol{\Sigma}$.

2. Se $\boldsymbol{Y} \sim t_{p}(\boldsymbol{\mu}, \boldsymbol{\Sigma}, \nu), c_{0}=\nu /(\nu-2), \mathrm{E}(\boldsymbol{Y})=\boldsymbol{\mu}$ e $\operatorname{Cov}(\boldsymbol{Y})=\nu /(\nu-2) \boldsymbol{\Sigma}$.

3. Se $\boldsymbol{Y} \sim E P_{p}(\boldsymbol{\mu}, \boldsymbol{\Sigma}, \nu)$,

$$
c_{0}=\frac{2^{(1+\nu) \Gamma\left[\frac{(p+2)(\nu+1)}{2}\right]}}{p \Gamma\left[\frac{p(\nu+1)}{2}\right]}
$$

e $\mathrm{E}(\boldsymbol{Y})=\boldsymbol{\mu}$ e $\operatorname{Cov}(\boldsymbol{Y})=c_{0} \boldsymbol{\Sigma}$. 


\section{Capítulo 3}

\section{Modelo de regressão beta com intercepto aleatório normal}

A classe dos modelos de regressão beta é usada para modelar variáveis medidas de forma continua que assumem valores no intervalo $(a, b)$. Estes modelos permitem modelar dados provenientes de taxas, proporções e frações, que geralmente são heteroscedásticos e assimétricos, e os parâmetros de regressão são interpretáveis em termos da média da variável resposta e não em termos de alguma transformação dos dados.

Nesse sentido, vários autores têm proposto modelos de regressão beta. Paolino (2001) propõe um modelo de regressão beta que modela a média e apresenta uma aplicação em pesquisas de ciências políticas. Kieschnick e McCullough (2003) propõem um modelo de regressão beta, que modela a média, como uma forma de realizar análise de regressão de variáveis observadas no intervalo (0,1). Ferrari e Cribari-Neto (2004) propõem um modelo de regressão beta, com uma parametrização da distribuição beta que é caracterizada pela média e por um parâmetro de precisão, que permite a modelagem da média considerando a precisão constante. Um modelo de regressão beta que modela a média e a precisão é proposto por Smithson e Verkuilen (2006).

A partir do modelo proposto por Ferrari e Cribari-Neto (2004) diversos trabalhos têm sido publicados. O trabalho de Venezuela (2008) considera equações de estimação generalizadas para os modelos de regressão beta com medidas repetidas, sob os enfoques da modelagem da média com homogeneidade da dispersão e da modelagem conjunta da média e da dispersão. O trabalho de Simas et al. (2010) considera uma estrutura de regressão para o parâmetro de precisão e uma estrutura de regressão não-linear para a média. Ferrari e Pinheiro (2011) desenvolvem dois testes de hipóteses baseados em estatísticas de razão de verossimilhanças ajustadas para modelos com estruturas de regressão lineares ou não lineares na média e na precisão. 
Um estudo detalhado para avaliar o impacto da especificação incorreta da função de ligação no modelo de regressão beta é apresentado em Andrade (2007). Zimprich (2010) apresenta um modelo de regressão beta com estrutura de regressão mista no parâmetro da média e ilustra a aplicabilidade do modelo na área da psicologia. Cribari-Neto e Zeileis (2010) desenvolvem o pacote betareg na linguagem de programação $R$ ( $R$ Development Core Team, 2012), que fornece estimativas de máxima verossimilhança para os parâmetros da classe dos modelos de regressão beta proposta por Ferrari e Cribari-Neto (2004) e estendida por Simas et al. (2010), além disso proporciona previsões para a média, o preditor linear, o parâmetro de precisão e a variância, assim como vários residuales e os testes de Wald dos coeficientes do modelo. Cribari-Neto e Souza (2012) adotam uma nova parametrização da distribuição beta e propõem um modelo de regressão beta com estrutura de regressão para a média e a dispersão, além disso mostram como realizar inferências sobre os parâmetros do submodelo da média sem ter que modelar a dispersão. Recentemente, Grün et al. (2012) apresentam extensões da regressão beta no pacote betareg como a redução e correção do viés nos estimadores de máxima verossimilhança, modelos de árvores de regressão beta e modelos de mistura finita. Ospina e Ferrari (2012) propõem uma classe de modelos de regressão beta que admite a presença de zeros e uns e apresentam estimação de máxima verossimilhança, métodos de diagnóstico e de seleção para essa classe de modelos, chamados modelos de regressão beta inflacionados.

Sob uma abordagem bayesiana, vários autores também têm realizado pesquisas no modelo de regressão beta. Buckley (2003) implementa o modelo de regressão beta proposto por Paolino (2001) por meio do método bayesiano Monte Carlo baseado em cadeias de Markov (MCMC). Branscum et al. (2007) apresentam um modelo de regressão hierárquico bayesiano para a análise de dados contínuos restritos ao intervalo $(0,1)$ e discutem metodologias para incorporar informação a priori na análise do modelo, ajustar o modelo proposto através do método MCMC e selecionar o modelo usando o fator de Bayes. Além disso estes autores apresentam um modelo de regressão beta semi-paramétrico usando splines penalizados.

Vários trabalhos sobre modelos de regressão beta com coeficientes de regressão aleatórios podem ser encontrados na literatura. Sob uma abordagem frequentista, Rigby e Stasinopoulos (2005) implementam o modelo de regressão beta com intercepto aleatório normal na estrutura de regressão da média e da dispersão assumindo a função de ligação logito. A estimação de parâmetros do modelo, fixando os componentes de variância, é feita no contexto dos modelos adivos generalizados para posição, escala e forma (GAMLSS) por meio da maximização da função de verossimilhança penalizada. Por sua vez, Verkuilen e Smithson (2012) propõem modelos de regressão beta com efeitos aleatórios normais na estrutura de regressão da média e da precisão assumindo a função de ligação logito. Os métodos de estimação usados são máxima verossimilhança marginal e estimação bayesiana. A aproximação das integrais nos dois métodos de estimação usados são quadratura de Gauss-Hermite e métodos 
de Monte Carlo via cadeias de Markov. Sob uma abordagem bayesiana, Bonat et al. (2012) propõem modelos de regressão beta com efeitos aleatórios na estrutura de regressão de média e desenvolvem inferência estatística por meio da maximização da verossimilhança marginal e da metodologia bayesiana de algoritmo data cloning. Figueroa-Zúñiga et al. (2012) estendem o modelo proposto por Ferrari e Cribari-Neto (2004) acrescentando efeitos aleatórios com distribuição normal e distribuição $t$-Student nas estruturas de regressão da média e precisão e discutem a especificação de distribuições a priori e a implementação computacional do modelo por meio do amostrador de Gibbs.

Neste capítulo estudamos um modelo denominado de modelo de regressão beta com intercepto aleatório normal. Desenvolvemos a estimação dos parâmetros do modelo e a predição dos interceptos aleatórios por meio do método de máxima verossimilhança marginal e da metodologia que considera o melhor preditor de Bayes empírico, respectivamente. Este modelo permite a modelagem conjunta da média e da dispersão da distribuição beta com parametrização dada em (2.8). No modelo assumimos várias funções de ligação para a média e a dispersão. Desemvolvemos também o pacote computacional BLMM na linguagem R para obter a estimação dos parâmetros e a predição dos interceptos aleatórios. Uma vantagem deste modelo é que permite descrever: a heterogeneidade de médias entre indivíduos mediante a inserção do intercepto aleatório na média e na dispersão entre observações repetidas num indivíduo por meio do intercepto aleatório no parâmetro de dispersão.

Na Seção 3.1 especificamos o modelo de regressão beta com intercepto aleatório normal e mostramos as expressões para a média, variância, covariância e correlação da distribuição marginal da variável resposta. Na Seção 3.2 apresentamos o método de estimação do modelo proposto. Na Seção 3.3 mostramos a metodologia da predição dos efeitos aleatórios. Por último, resultados de simulações avaliando o processo de estimação proposto e as conclusões são apresentados nas Seções 3.4 e 3.5, respectivamente.

\subsection{Modelo proposto}

As características principais do modelo beta com intercepto aleatório normal são desenvolvidas com detalhes nesta seção. Sem perda de generalidade, vamos a supor $(0,1)=(a, b)$. No caso em que a variável resposta é definida no intervalo $(a, b)$ modelamos $(y-a) /(b-a)$ no lugar de $y$.

Seja $y_{i j}$ o valor da variável resposta para o $i$-ésimo indivíduo no tempo $t_{i j}, i=1, \ldots, N$, $j=1, \ldots, n_{i}$ e seja $\boldsymbol{y}_{i}=\left(y_{i 1}, y_{i 2}, \ldots, y_{i n_{i}}\right)^{\top}$ o vetor com as $n_{i}$ observações repetidas do $i$ ésimo indivíduo. Assumimos que, condicional aos interceptos aleatórios $\gamma_{i 1}$ e $\gamma_{i 2}$, as respostas $y_{i 1}, y_{i 2}, \ldots, y_{i n_{i}}$ são independentes e cada uma segue uma distribuição beta com função den- 
sidade de probabilidade dada em (2.8). Os interceptos aleatórios $\gamma_{i 1}$ e $\gamma_{i 2}$ são independientes e cada um segue uma distribuição normal com média zero e desvio padrão $\lambda_{1}$ e $\lambda_{2}$, respectivamente. Assumimos também que os interceptos aleatórios $\gamma_{i 1}$ e $\gamma_{i 2}$ são independentes entre si. O modelo beta com intercepto aleatório normal pode ser escrito como

$$
\begin{aligned}
y_{i j} \mid \gamma_{i 1}, \gamma_{i 2} & \stackrel{\text { ind }}{\sim} B e\left(\mu_{i j}, \sigma_{i j}\right), \\
\gamma_{i 1} & \stackrel{\text { i.i.d }}{\sim} N\left(0, \lambda_{1}^{2}\right), \\
\gamma_{i 2} & \stackrel{\text { i.i.d. }}{\sim} N\left(0, \lambda_{2}^{2}\right),
\end{aligned}
$$

para $i=1,2, \ldots, N$ e $j=1,2, \ldots, n_{i}$, em que $\mathrm{E}\left(y_{i j} \mid \gamma_{i 1}, \gamma_{i 2}\right)=\mu_{i j}$ e $\operatorname{Var}\left(y_{i j} \mid \gamma_{i 1}, \gamma_{i 2}\right)=$ $\sigma_{i j}^{2} \mu_{i j}\left(1-\mu_{i j}\right)$. Os parâmetros $\mu_{i j}$ e $\sigma_{i j}$ satisfazem as seguintes relações funcionais

$$
\begin{aligned}
& g_{1}\left(\mu_{i j}\right)=\eta_{i j 1}=\boldsymbol{x}_{i j 1}^{\top} \boldsymbol{\beta}_{1}+\gamma_{i 1} \\
& g_{2}\left(\sigma_{i j}\right)=\eta_{i j 2}=\boldsymbol{x}_{i j 2}^{\top} \boldsymbol{\beta}_{2}+\gamma_{i 2},
\end{aligned}
$$

em que $\boldsymbol{x}_{i j 1}=\left(x_{i j 11}, x_{i j 21}, \ldots, x_{i j p_{1} 1}\right)^{\top}$ e $\boldsymbol{x}_{i j 2}=\left(x_{i j 12}, x_{i j 22}, \ldots, x_{i j p_{2} 2}\right)^{\top}$ contêm valores das variáveis explicativas associadas aos efeitos fixos, $\boldsymbol{\beta}_{1}=\left(\beta_{11}, \beta_{21}, \ldots, \beta_{p_{1} 1}\right)^{\top}$ e $\boldsymbol{\beta}_{2}=$ $\left(\beta_{12}, \beta_{22}, \ldots, \beta_{p_{2}}\right)^{\top}$ contêm os parâmetros desconhecidos fixos e $\gamma_{i 1}$ e $\gamma_{i 2}$ são os interceptos aleatórios associados ao $i$-ésimo indivíduo.

Assumimos que as funções de ligação $g_{1}:(0,1) \rightarrow \mathbb{R}$ e $g_{2}:(0,1) \rightarrow \mathbb{R}$ são funções conhecidas, estritamente monótonas e duas vezes diferenciáveis. Para o modelo proposto podemos usar a mesma ou uma diferente função de ligação para $\mu$ e $\sigma$. Na Tabela 3.1 são apresentados exemplos de funções de ligação e suas funções inversas para o parâmetro $\mu$. Estas funções de ligação podem também ser utilizadas na estrutura de regressão de $\sigma$. Uma discussão sobre o uso de algumas destas funções de ligação em modelos lineares generalizados pode ser encontrada em McCullagh e Nelder (1983, p. 107) e um estudo sobre os efeitos da especificação incorreta da função de ligação no modelo de regressão beta encontra-se descrito em Andrade (2007).

Seguindo a abordagem marginal apresentada para o caso do modelo linear normal de efeitos mistos é possível obter a média, a variância, a covariância e a correlação da distribuição marginal de $y_{i j}$, descritas a seguir em detalhes.

\subsubsection{Média}

A média marginal de $y_{i j}$ pode ser calculada com base na esperança condicional de $y_{i j}$ dados os efeitos aleatórios $\gamma_{i 1}$ e $\gamma_{i 2}$, ou seja,

$$
\mathrm{E}\left(y_{i j}\right)=\mathrm{E}\left[\mathrm{E}\left(y_{i j} \mid \gamma_{i 1}, \gamma_{i 2}\right)\right]=\mathrm{E}\left(\mu_{i j}\right)
$$


Tabela 3.1: Funções de ligação.

\begin{tabular}{lcc}
\hline Função de ligação & $g(\mu)$ & $\mu$ \\
\hline logito & $\log \{\mu /(1-\mu)\}$ & $\exp \left(\eta_{1}\right) /\{1+\exp (\eta)\}$ \\
probito & $\Phi^{-1}(\mu)$ & $\Phi(\eta)$ \\
complemento log-log & $\log \{-\log (1-\mu)\}$ & $1-\exp \{-\exp (\eta)\}$ \\
$\operatorname{log-log}$ & $-\log \{-\log (\mu)\}$ & $\exp \{-\exp (-\eta)\}$ \\
Cauchy & $\tan \{\pi(\mu-0,5)\}$ & $\{\arctan (\eta) / \pi\}+0,5$ \\
& & \\
\hline
\end{tabular}

A esperança (3.3) é calculada com relação à distribuição do efeito aleatório $\gamma_{i 1}$. Se usamos, por exemplo, a função de ligação logito na estrutura de regressão de $\mu$ e $\sigma$ a média marginal de $y_{i j}$ é dada por

$$
\begin{aligned}
\mathrm{E}\left(y_{i j}\right) & =\mathrm{E}\left(\frac{e^{\eta_{i j 1}}}{1+e^{\eta_{i j 1}}}\right) \\
& =\mathrm{E}\left(\frac{e^{\boldsymbol{x}_{i j 1}^{\top} \boldsymbol{\beta}_{1}+\gamma_{i 1}}}{1+e^{\boldsymbol{x}_{i j 1}^{\top} \boldsymbol{\beta}_{1}+\gamma_{i 1}}}\right) \\
& =\mathrm{E}\left(\frac{e^{\boldsymbol{x}_{i j 1}^{\top} \boldsymbol{\beta}_{1}} e^{\gamma_{i 1}}}{1+e^{\boldsymbol{x}_{i j 1}^{\top} \boldsymbol{\beta}_{1}} e^{\gamma_{i 1}}}\right) .
\end{aligned}
$$

Se escrevemos $a=e^{\boldsymbol{x}_{i j 1}^{\top} \boldsymbol{\beta}_{1}}$, obtemos

$$
\begin{aligned}
\mathrm{E}\left(y_{i j}\right) & =\mathrm{E}\left(\frac{a e^{\gamma_{i 1}}}{1+a e^{\gamma_{i 1}}}\right) \\
& =\mathrm{E}\left(1-\frac{1}{1+a e^{\gamma_{i 1}}}\right) \\
& =1-\mathrm{E}\left(\frac{1}{1+a e^{\gamma_{i 1}}}\right) .
\end{aligned}
$$

\subsubsection{Variância}

Para calcular a variância marginal de $y_{i j}$ usamos a propriedade da variância condicional dada por

$$
\operatorname{Var}(Y)=\operatorname{Var}[\mathrm{E}(Y \mid X)]+\mathrm{E}[\operatorname{Var}(Y \mid X)]
$$


sendo $X$ e $Y$ duas variáveis aleatórias. Utilizando esta expressão obtemos

$$
\begin{aligned}
\operatorname{Var}\left(y_{i j}\right) & =\operatorname{Var}\left[\mathrm{E}\left(y_{i j} \mid \gamma_{i 1}, \gamma_{i 2}\right)\right]+\mathrm{E}\left[\operatorname{Var}\left(y_{i j} \mid \gamma_{i 1}, \gamma_{i 2}\right)\right] \\
& =\operatorname{Var}\left(\mu_{i j}\right)+\mathrm{E}\left[\sigma_{i j}^{2} \mu_{i j}\left(1-\mu_{i j}\right)\right]
\end{aligned}
$$

como os efeitos aleatórios associados à média e à dispersão são independentes então

$$
\operatorname{Var}\left(y_{i j}\right)=\mathrm{E}\left(\mu_{i j}^{2}\right)+\left(\mathrm{E}\left(\mu_{i j}\right)\right)^{2}+\mathrm{E}\left(\sigma_{i j}^{2}\right) \cdot \mathrm{E}\left(\mu_{i j}\left(1-\mu_{i j}\right)\right)
$$

Podemos notar que a expressão da variância de $y_{i j}$ é composta por três parcelas. As duas primeiras correspondem a esperanças calculadas em relação à distribuição do efeito aleatório $\gamma_{i 1}$ e a terceira corresponde ao produto entre duas esperanças, $\mathrm{E}\left(\sigma_{i j}^{2}\right)$ e $\mathrm{E}\left(\mu_{i j}\left(1-\mu_{i j}\right)\right)$, obtidas em relação às distribuições dos efeitos aleatórios $\gamma_{i 2}$ e $\gamma_{i 1}$, respectivamente.

Considerando a função de ligação logito para $\mu$ e $\sigma$, por exemplo, e a expressão (3.5) e escrevendo $e^{\eta_{i j 1}}=e^{\boldsymbol{x}_{i j 1}^{\top} \boldsymbol{\beta}_{1}} e^{\gamma_{i 1}}=a e^{\gamma_{i 1}}$ e $e^{\eta_{i j 2}}=e^{\boldsymbol{x}_{i j 2}^{\top} \boldsymbol{\beta}_{2}} e^{\gamma_{i 2}}=b e^{\gamma_{i 2}}$, com $b=e^{\boldsymbol{x}_{i j 2}^{\top} \boldsymbol{\beta}_{2}}$, temos que a variância de $y_{i j}$ é dada por

$$
\begin{aligned}
\operatorname{Var}\left(y_{i j}\right) & =\operatorname{Var}\left(\frac{a e^{\gamma_{i 1}}}{1+a e^{\gamma_{i 1}}}\right)+\mathrm{E}\left(\left(\frac{b e^{\gamma_{i 2}}}{1+b e^{\gamma_{i 2}}}\right)^{2} \frac{a e^{\gamma_{i 1}}}{1+a e^{\gamma_{i 1}}} \frac{1}{1+a e^{\gamma_{i 1}}}\right) \\
& =\operatorname{Var}\left(1-\frac{1}{1+a e^{\gamma_{i 1}}}\right)+\mathrm{E}\left(\left(\frac{b e^{2 \gamma_{i 2}}}{1+b e^{\gamma_{i 2}}}\right)^{2} \frac{a e^{\gamma_{i 1}}}{\left(1+a e^{\gamma_{i 1}}\right)^{2}}\right) \\
& =\operatorname{Var}\left(\frac{1}{1+a e^{\gamma_{i 1}}}\right)+\mathrm{E}\left[\left(\frac{b e^{\gamma_{i 2}}}{1+b e^{\gamma_{i 2}}}\right)^{2}\right] \mathrm{E}\left[\frac{a e^{\gamma_{i 1}}}{\left(1+a e^{\gamma_{i 1}}\right)^{2}}\right] \\
& =\mathrm{E}\left(\frac{1}{\left(1+a e^{\gamma_{i 1}}\right)^{2}}\right)-\left[\mathrm{E}\left(\frac{1}{1+a e^{\gamma_{i 1}}}\right)\right]^{2}+\mathrm{E}\left(\frac{b^{2} e^{2 \gamma_{i 2}}}{\left(1+b e^{\gamma_{i 2}}\right)^{2}}\right) \mathrm{E}\left(\frac{a e^{\gamma_{i 1}}}{\left(1+a e^{\gamma_{i 1}}\right)^{2}}\right) \\
& =\mathrm{E}\left(\frac{1}{\left(1+a e^{\gamma_{i 1}}\right)^{2}}\right)-\left[\mathrm{E}\left(\frac{1}{1+a e^{\gamma_{i 1}}}\right)\right]^{2}+a b^{2} \mathrm{E}\left(\frac{e^{2 \gamma_{i 2}}}{\left(1+b e^{\gamma_{i 2}}\right)^{2}}\right) \mathrm{E}\left(\frac{e^{\gamma_{i 1}}}{\left(1+a e^{\gamma_{i 1}}\right)^{2}}\right) .
\end{aligned}
$$

\subsubsection{Covariância e correlação}

Assumindo independência condicional dos $y_{i j}$ e usando a definição da covariância condicional dada por

$$
\operatorname{Cov}(Y, W)=\operatorname{Cov}(\mathrm{E}(Y \mid X), \mathrm{E}(W \mid X))+\mathrm{E}[\operatorname{Cov}(Y, W \mid X)]
$$

sendo $X, Y$ e $W$ variáveis aleatórias, temos que a covariância entre $y_{i j}$ e $y_{i j^{\prime}}$, duas observações nos momentos $j$ e $j^{\prime}$ do $i$-ésimo indivíduo, é dada por

$$
\operatorname{Cov}\left(y_{i j}, y_{i j^{\prime}}\right)=\operatorname{Cov}\left(\mathrm{E}\left(y_{i j} \mid \gamma_{i 1}, \gamma_{i 2}\right), \mathrm{E}\left(y_{i j^{\prime}} \mid \gamma_{i 1}, \gamma_{i 2}\right)\right)+\mathrm{E}\left[\operatorname{Cov}\left(y_{i j}, y_{i j^{\prime}} \mid \gamma_{i 1}, \gamma_{i 2}\right)\right]
$$


em que o termo $\mathrm{E}\left[\operatorname{Cov}\left(y_{i j}, y_{i j^{\prime}} \mid \gamma_{i 1}, \gamma_{i 2}\right)\right]$ é igual a zero pela independência condicional das observações e, portanto, a covariância de $y_{i j}$ é dada por

$$
\begin{aligned}
\operatorname{Cov}\left(y_{i j}, y_{i j^{\prime}}\right) & =\operatorname{Cov}\left(\mathrm{E}\left(y_{i j} \mid \gamma_{i 1}, \gamma_{i 2}\right), \mathrm{E}\left(y_{i j^{\prime}} \mid \gamma_{i 1}, \gamma_{i 2}\right)\right) \\
& =\operatorname{Cov}\left(\mu_{i j}, \mu_{i j^{\prime}}\right) \\
& =\mathrm{E}\left(\mu_{i j}, \mu_{i j^{\prime}}\right)-\mathrm{E}\left(\mu_{i j}\right) \cdot \mathrm{E}\left(\mu_{i j^{\prime}}\right)
\end{aligned}
$$

Usando a função de ligação logito para $\mu$ e $\sigma$, por exemplo, e (3.7) podemos escrever a covariância de $y_{i j}$ como

$$
\begin{aligned}
\operatorname{Cov}\left(y_{i j}, y_{i j^{\prime}}\right) & =\operatorname{Cov}\left(\frac{e^{\eta_{i j 1}}}{1+e^{\eta_{i j 1}}}, \frac{e^{\eta_{i j^{\prime} 1}}}{1+e^{\eta_{i j^{\prime} 1}}}\right) \\
& =\operatorname{Cov}\left(1-\frac{1}{1+e^{\eta_{i j 1}}}, 1-\frac{1}{1+e^{\eta_{i j^{\prime} 1}}}\right)
\end{aligned}
$$

e usando as propriedades da covariância: $\operatorname{Cov}(a+X, b+Y)=\operatorname{Cov}(X, Y)$ e $\operatorname{Cov}(a X, b Y)=$ $a b \operatorname{Cov}(X, Y)$, temos que (3.8) fica dada por

$$
\begin{aligned}
\operatorname{Cov}\left(y_{i j}, y_{i j^{\prime}}\right) & =\mathrm{E}\left(\frac{1}{1+e^{\eta_{i j 1}}} \frac{1}{1+e^{\eta_{i j^{\prime} 1}}}\right)-\mathrm{E}\left(\frac{1}{1+e^{\eta_{i j 1}}}\right) \mathrm{E}\left(\frac{1}{1+e^{\eta_{i j^{\prime} 1}}}\right) \\
& =\mathrm{E}\left(\frac{1}{1+e^{\eta_{i j 1}}} \frac{1}{1+e^{\eta_{i j^{\prime} 1}}}\right)-\left(1-\mathrm{E}\left(y_{i j}\right)\right)\left(1-\mathrm{E}\left(y_{i j^{\prime}}\right)\right) .
\end{aligned}
$$

Finalmente, a correlação entre as duas observações $y_{i j}$ e $y_{i j^{\prime}}$ pode ser calculada como segue

$$
\operatorname{Corr}\left(y_{i j}, y_{i j^{\prime}}\right)=\frac{\operatorname{Cov}\left(y_{i j}, y_{i j^{\prime}}\right)}{\sqrt{\operatorname{Var}\left(y_{i j}\right) \operatorname{Var}\left(y_{i j^{\prime}}\right)}}
$$

As integrais envolvidas nos valores esperados nas expressões (3.3) a (3.10) não tem forma fechada e devem ser resolvidas por métodos de integração numérica. Nota-se que as expressões (3.4), (3.6), (3.9) e (3.10) são gerais, isto é, não dependem da distribuição suposta dos efeitos aleatórios. As expressões da média e variância da distribuição marginal de $y_{i j}$ serão utilizadas no Capítulo 6.

\subsection{Estimação}

A inferência estatística proposta neste trabalho para o modelo de regressão beta com intercepto aleatório normal está baseada no método de máxima verossimilhança.

Considerando $\boldsymbol{\theta}=\left(\boldsymbol{\beta}_{1}^{\top}, \boldsymbol{\beta}_{2}^{\top}, \lambda_{1}, \lambda_{2}\right)^{\top}$ o vetor de parâmetros a ser estimado no espaço paramétrico

$$
\boldsymbol{\Theta}=\left\{\boldsymbol{\theta} \in \mathbb{R}^{p} \mid \boldsymbol{\beta}_{1} \in \mathbb{R}^{p_{1}}, \boldsymbol{\beta}_{2} \in \mathbb{R}^{p_{2}}, \lambda_{1} \in \mathbb{R}^{+}, \lambda_{2} \in \mathbb{R}^{+}\right\}
$$


em que $p=p_{1}+p_{2}+2$, temos que a distribuição marginal de $\boldsymbol{y}_{i}=\left(y_{1 i}, \ldots, y_{n_{i}}\right)^{\top}$ é dada por

$$
f\left(\boldsymbol{y}_{i} ; \boldsymbol{\theta}\right)=\int_{\mathbb{R}^{2}} \prod_{j=1}^{n_{i}} f\left(y_{i j} \mid \gamma_{i 1}, \gamma_{i 2} ; \boldsymbol{\beta}_{1}, \boldsymbol{\beta}_{2}\right) \cdot f\left(\gamma_{i 1} ; \lambda_{1}\right) f\left(\gamma_{i 2} ; \lambda_{2}\right) d \gamma_{i 1} d \gamma_{i 2},
$$

e a função de verossimilhança para as observações $\boldsymbol{y}=\left(\boldsymbol{y}_{1}, \ldots, \boldsymbol{y}_{N}\right)^{\top}$ é da forma

$$
\begin{aligned}
L(\boldsymbol{\theta}) & =\prod_{i=1}^{N} f\left(\boldsymbol{y}_{i} ; \boldsymbol{\theta}\right) \\
& =\prod_{i=1}^{N} \int_{\mathbb{R}^{2}} \prod_{j=1}^{n_{i}} f\left(y_{i j} \mid \gamma_{i 1}, \gamma_{i 2} ; \boldsymbol{\beta}_{1}, \boldsymbol{\beta}_{2}\right) \cdot f\left(\gamma_{i 1} ; \lambda_{1}\right) f\left(\gamma_{i 2} ; \lambda_{2}\right) d \gamma_{i 1} d \gamma_{i 2}
\end{aligned}
$$

em que $f\left(y_{i j} \mid \gamma_{i 1}, \gamma_{i 2} ; \boldsymbol{\beta}_{1}, \boldsymbol{\beta}_{2}\right)$ é a função densidade de probabilidade condicional de $y_{i j}$ da$\operatorname{dos} \gamma_{i 1}, \gamma_{i 2}$ e $f\left(\gamma_{i 1} ; \lambda_{1}\right)$ e $f\left(\gamma_{i 2} ; \lambda_{2}\right)$ são as funções densidades de probabilidades de $\gamma_{i 1}$ e $\gamma_{i 2}$, respectivamente.

Ao contrário dos modelos lineares mistos normais, a densidade marginal (3.11) e a função de verossimilhança (3.12) não têm expressões analíticas. A dificuldade na inferência baseada no método de máxima verossimilhança é a avaliação das integrais na função de verossimilhança (3.12). Os métodos que são geralmente usados para resolver essa dificuldade incluem métodos de integração de Monte Carlo, algoritmos EM e métodos aproximados, (Wu, 2010). Os denominados métodos de Monte Carlo usam quadratura de Gauss-Hermite ou técnicas de integração de Monte Carlo para aproximar a integral na função de verossimilhança (3.12). Por outro lado, os algoritmos EM maximizam indiretamente a verossimilhança usando métodos numéricos estocásticos ou de Monte Carlo no passo E. Finalmente, os métodos aproximados são chamados assim por usar expansões de Taylor ou aproximações de Laplace para aproximar diretamente a função de verossimilhança.

Para a avaliação das integrais envolvidas na função de verossimilhança (3.12) utilizamos o método de integração numérica de Gauss-Hermite multidimensional descrito na Seção 2.3. Na função de verossimilhança (3.12) precisamos avaliar a seguinte integral

$$
\begin{aligned}
I_{i} & =\int_{\mathbb{R}^{2}} \prod_{j=1}^{n_{i}} f\left(y_{i j} \mid \gamma_{i 1}, \gamma_{i 2} ; \boldsymbol{\beta}_{1}, \boldsymbol{\beta}_{2}\right) \cdot f\left(\gamma_{i 1} ; \lambda_{1}\right) f\left(\gamma_{i 2} ; \lambda_{2}\right) d \gamma_{i 1} d \gamma_{i 2} \\
& =\int_{\mathbb{R}^{2}} \prod_{j=1}^{n_{i}} f\left(y_{i j} \mid \gamma_{i 1}, \gamma_{i 2} ; \boldsymbol{\beta}_{1}, \boldsymbol{\beta}_{2}\right) \frac{e^{-\frac{\gamma_{i 1}^{2}}{2 \lambda_{1}^{2}}}}{\sqrt{2 \pi} \lambda_{1}} \frac{e^{-\frac{\gamma_{i 2}^{2}}{2 \lambda_{2}^{2}}}}{\sqrt{2 \pi} \lambda_{2}} d \gamma_{i 1} d \gamma_{i 2} .
\end{aligned}
$$

Fazendo a seguinte mudança de variáveis

$$
b_{i 1}=\frac{\gamma_{i 1}}{\sqrt{2} \lambda_{1}}, \quad b_{i 2}=\frac{\gamma_{i 2}}{\sqrt{2} \lambda_{2}}
$$


obtemos que a integral $I_{i}$ é dada por

$$
I_{i}=\int_{\mathbb{R}^{2}} \prod_{j=1}^{n_{i}} f\left(y_{i j} \mid \sqrt{2} \lambda_{1} b_{i 1}, \sqrt{2} \lambda_{2} b_{i 2} ; \boldsymbol{\beta}_{1}, \boldsymbol{\beta}_{2}\right) \frac{\exp \left(-b_{i 1}^{2}\right) \exp \left(-b_{i 2}^{2}\right)}{\pi} d b_{i 1} d b_{i 2}
$$

que tem a forma (2.4). Usando o método da quadratura de Gauss-Hermite multidimensional, a integral $I_{i}$ pode ser aproximada como

$$
I_{i} \cong \sum_{k_{1}=1}^{Q_{1}} \sum_{k_{2}=1}^{Q_{2}} \prod_{j=1}^{n_{i}} f\left(y_{i j} \mid \sqrt{2} \lambda_{1} z_{k_{1}}, \sqrt{2} \lambda_{2} z_{k_{2}} ; \boldsymbol{\beta}_{1}, \boldsymbol{\beta}_{2}\right) \frac{w_{k_{1}} w_{k_{2}}}{\pi}
$$

em que $z_{k_{1}}$ e $z_{k_{2}}$ são os pontos de quadratura e $w_{k_{1}}$ e $w_{k_{2}}$ são os pesos correspondentes. Portanto, a função de verossimilhança aproximada é dada por

$$
L(\boldsymbol{\theta}) \cong \prod_{i=1}^{N}\left(\sum_{k_{1}=1}^{Q_{1}} \sum_{k_{2}=1}^{Q_{2}} \prod_{j=1}^{n_{i}} f\left(y_{i j} \mid \sqrt{2} \lambda_{1} z_{k_{1}}, \sqrt{2} \lambda_{2} z_{k_{2}} ; \boldsymbol{\beta}_{1}, \boldsymbol{\beta}_{2}\right) \frac{w_{k_{1}} w_{k_{2}}}{\pi}\right)
$$

e o logaritmo da função de verossimilhança $\ell(\boldsymbol{\theta})$ pode ser escrito como

$$
\ell(\boldsymbol{\theta}) \cong \sum_{i=1}^{N} \log \left(\sum_{k_{1}=1}^{Q_{1}} \sum_{k_{2}=1}^{Q_{2}} \prod_{j=1}^{n_{i}} f\left(y_{i j} \mid \sqrt{2} \lambda_{1} z_{k_{1}}, \sqrt{2} \lambda_{2} z_{k_{2}} ; \boldsymbol{\beta}_{1}, \boldsymbol{\beta}_{2}\right) \frac{w_{k_{1}} w_{k_{2}}}{\pi}\right) .
$$

A função escore aproximada, obtida diferenciando o logaritmo da função de verossimilhança $\ell(\boldsymbol{\theta})$ em relação a cada um dos parâmetros é dada por $\boldsymbol{U}(\boldsymbol{\theta})=\left(\boldsymbol{U}_{\boldsymbol{\beta}_{1}}^{\top}(\boldsymbol{\theta}), \boldsymbol{U}_{\boldsymbol{\beta}_{2}}^{\top}(\boldsymbol{\theta})\right.$, $\left.U_{\lambda_{1}}(\boldsymbol{\theta}), U_{\lambda_{2}}\right)^{\top}$. No Apêndice A.1 são apresentadas as expressões para os componentes do vetor $\boldsymbol{U}(\boldsymbol{\theta})$.

O estimador de máxima verossimilhança de $\boldsymbol{\theta}=\left(\boldsymbol{\beta}_{1}^{\top}, \boldsymbol{\beta}_{2}^{\top}, \lambda_{1}, \lambda_{2}\right)^{\top}$ é obtido como a solução do sistema de equações $\boldsymbol{U}(\boldsymbol{\theta})=\mathbf{0}$. Podemos notar que os estimadores dos componentes de $\boldsymbol{\theta}$ não têm uma expressão fechada e precisam ser obtidos numericamente maximizando (3.16).

Sob as condições usuais de regularidade, o estimador de máxima verossimilhança, $\hat{\boldsymbol{\theta}}$, é assintoticamente normal com média $\boldsymbol{\theta}$ e matriz de variância-covariância $\boldsymbol{J}^{-\mathbf{1}}(\boldsymbol{\theta})$, isto é

$$
\sqrt{n}(\hat{\boldsymbol{\theta}}-\boldsymbol{\theta}) \rightarrow N\left(\mathbf{0}, \boldsymbol{J}^{-\mathbf{1}}(\boldsymbol{\theta})\right)
$$

em que $\boldsymbol{J}(\boldsymbol{\theta})$ é a matriz de informação observada, obtida como $\boldsymbol{J}(\boldsymbol{\theta})=-\partial^{2} \ell(\boldsymbol{\theta}) / \partial \boldsymbol{\theta} \partial \boldsymbol{\theta}^{\top}$. Os elementos da matriz $\boldsymbol{J}(\boldsymbol{\theta})$ são apresentados no Apêndice A.2. Para obter os desvios padrões precisa-se obter também de forma numérica as estimativas dos elementos de $\boldsymbol{J}(\boldsymbol{\theta})$. 


\section{Aspectos computacionais}

Organizamos a proposta implementando um pacote específico no R chamado BLMM que permite obter as estimativas de máxima verossimilhança do modelo proposto por meio da função blmmML. Implementamos também a função GHQ que calcula os pontos e pesos de quadratura eliminando combinações de pontos de quadratura que contribuem pouco à aproximação das integrais; para maiores detalhes da função, ver Hernández et al. (2013). As funções nlminb e optim do pacote stats são usadas para maximizar a função (3.16). Assumimos que o número de pontos de quadratura, $Q_{1}$ e $Q_{2}$, são iguais. No Capítulo 8 descrevemos em detalhes estas funções.

As funções nlminb e optim usadas na maximização do logaritmo de função de verossimilhança precisam da especificação de valores iniciais para os parâmetros. Como valores

inicias de $\boldsymbol{\beta}_{1}^{\top}$ e $\boldsymbol{\beta}_{2}^{\top}$ sugerimos obter $\hat{\boldsymbol{\beta}}_{1}^{\top}$ and $\hat{\boldsymbol{\beta}}_{2}^{\top}$ como as estimativas do modelo de regressão beta sem efeito aleatório, ou seja, considerando

$$
\begin{aligned}
& g_{1}\left(\mu_{i j}\right)=\boldsymbol{x}_{i j 1}^{\top} \boldsymbol{\beta}_{1} \\
& g_{2}\left(\sigma_{i j}\right)=\boldsymbol{x}_{i j 2}^{\top} \boldsymbol{\beta}_{2},
\end{aligned}
$$

sendo que este pode ser ajustado, por exemplo, no pacote gamlss de Rigby e Stasinopoulos (2005) ou betareg de Cribari-Neto e Zeileis (2010) do R. Para os parâmetros $\lambda_{1}$ e $\lambda_{2}$ sugerimos usar valores de 0,5. Em modelos de regressão ordinal mistos Hedeker e Gibbons (1994) sugerem usar uma matriz diagonal como valor inicial da matriz de covariância dos efeitos aleatórios, com elementos da matriz diagonal iguais a uma fração do valor da variância residual, e em modelos lineares generalizados mistos Brostöm e Holmberg (2011) sugerem valores de 0,5 para a variabilidade do intercepto aleatório, neste caso $\lambda_{1}=0,5$ e $\lambda_{2}=0,5$.

\subsection{Predição dos efeitos aleatórios}

Embora na prática o interesse principal seja a estimação dos parâmetros da distribuição marginal de $\boldsymbol{y}_{i}$, muitas vezes é útil também obter as predições dos efeitos aleatórios. Segundo Molenberghs e Verbeke (2005) essas predições refletem a variabilidade entre indivíduos e permitem a detecção de perfis especiais, por exemplo, indivíduos atípicos ou grupos de indivíduos que evoluem de forma diferente no tempo. Além disso, as predições dos efeitos aleatórios são necessárias se queremos obter predições dos perfis individuais.

No contexto dos modelos lineares de efeitos aleatórios e dos modelos lineares generalizados mistos para dados longitudinais, Verbeke e Molenberghs (2009) e Fitzmaurice et al. (2009) notam que a abordagem predominante para atribuir valores aos efeitos aleatórios é a metodologia que considera o melhor preditor de Bayes empírico. Para o modelo linear normal 
de efeitos mistos, por exemplo, a estimação dos efeitos aleatórios é baseada na distribuição a posteriori $f\left(\boldsymbol{b}_{i} \mid \boldsymbol{y}_{i}, \boldsymbol{\beta}, \boldsymbol{\Sigma}\right)$ dada por

$$
f\left(\boldsymbol{b}_{i} \mid \boldsymbol{y}_{i}, \boldsymbol{\beta}, \boldsymbol{\Sigma}\right)=\frac{f\left(\boldsymbol{y}_{i} \mid \boldsymbol{b}_{i}, \boldsymbol{\beta}\right) f\left(\boldsymbol{b}_{i}, \boldsymbol{\Sigma}\right)}{\int_{\mathbb{R}^{q}} f\left(\boldsymbol{y}_{i} \mid \boldsymbol{b}_{i}, \boldsymbol{\beta}\right) f\left(\boldsymbol{b}_{i}, \boldsymbol{\Sigma}\right) d \boldsymbol{b}_{i}},
$$

Geralmente, $\boldsymbol{b}_{i}$ é predito por meio da média da distribuição a posteriori, chamada de média a posteriori de $\boldsymbol{b}_{i}$. Este preditor é denominado melhor preditor de Bayes empírico e é dado por

$$
\begin{aligned}
\widetilde{\boldsymbol{b}}_{i} & =\mathrm{E}\left[\boldsymbol{b}_{i} \mid \boldsymbol{y}_{i}\right] \\
& =\int_{\mathbb{R}^{q}} \boldsymbol{b}_{i} f\left(\boldsymbol{b}_{i} \mid \boldsymbol{y}_{i}, \boldsymbol{\beta}, \boldsymbol{\Sigma}\right) d \boldsymbol{b}_{i} .
\end{aligned}
$$

Para o modelo de regressão beta com intercepto aleatório normal propomos usar essa abordagem para obter o melhor preditor dos interceptos aleatórios como segue:

$$
\begin{gathered}
\widetilde{\gamma}_{i 1}=\mathrm{E}\left[\gamma_{i 1} \mid y_{i j} ; \hat{\boldsymbol{\theta}}\right]=\frac{\int_{\mathbb{R}^{2}} \gamma_{i 1} \prod_{j=1}^{n i} f\left(y_{i j} \mid \gamma_{i 1}, \gamma_{i 2} ; \hat{\boldsymbol{\beta}}_{1}, \hat{\boldsymbol{\beta}}_{2}\right) f\left(\gamma_{i 1} ; \hat{\lambda}_{1}\right) f\left(\gamma_{i 2} ; \hat{\lambda}_{2}\right) d \gamma_{i 1} d \gamma_{i 2}}{\int_{\mathbb{R}^{2}} \prod_{j=1}^{n i} f\left(y_{i j} \mid \gamma_{i 1}, \gamma_{i 2} ; \hat{\boldsymbol{\beta}}_{1}, \hat{\boldsymbol{\beta}}_{2}\right) f\left(\gamma_{i 1} ; \hat{\lambda}_{1}\right) f\left(\gamma_{i 2} ; \hat{\lambda}_{2}\right) d \gamma_{i 1} d \gamma_{i 2}}, \\
\widetilde{\gamma}_{i 2}=\mathrm{E}\left[\gamma_{i 2} \mid y_{i j} ; \hat{\boldsymbol{\theta}}\right]=\frac{\int_{\mathbb{R}^{2}} \gamma_{i 2} \prod_{j=1}^{n i} f\left(y_{i j} \mid \gamma_{i 1}, \gamma_{i 2} ; \hat{\boldsymbol{\beta}}_{1}, \hat{\boldsymbol{\beta}}_{2}\right) f\left(\gamma_{i 1} ; \hat{\lambda}_{1}\right) f\left(\gamma_{i 2} ; \hat{\lambda}_{2}\right) d \gamma_{i 1} d \gamma_{i 2}}{\int_{\mathbb{R}^{2}} \prod_{j=1}^{n i} f\left(y_{i j} \mid \gamma_{i 1}, \gamma_{i 2} ; \hat{\boldsymbol{\beta}}_{1}, \hat{\boldsymbol{\beta}}_{2}\right) f\left(\gamma_{i 1} ; \hat{\lambda}_{1}\right) f\left(\gamma_{i 2} ; \hat{\lambda}_{2}\right) d \gamma_{i 1} d \gamma_{i 2}},
\end{gathered}
$$

em que $i=1,2, \ldots, N$ e $j=1,2, \ldots, n_{i}$. As integrais no numerador e denominador de (3.18) e (3.19) são resolvidas por meio da quadratura de Gauss-Hermite, da mesma forma que a integral (3.13). A predição dos efeitos aleatórios foi implementada na função re • prediction do pacote BLMM descrita em detalhes no Capítulo 8.

A metodologia de estimação e predição proposta permite obter por meio de (3.17) as estimativas dos desvios padrões de $\lambda_{1}$ e $\lambda_{2}$. Estas estimativas são úteis para a construção de intervalos de confiança de $\lambda_{1}$ e $\lambda_{2}$.

\subsection{Estudo de simulação}

Nesta seção apresentamos um estudo de simulação para o modelo proposto na seção 3.1 a fim de descrever o desempenho do processo de estimação quando aumentamos o número de indivíduos, o número de observações por indivíduo e o desvio padrão dos interceptos aleatórios. 
Os dados são gerados de acordo com o seguinte modelo

$$
\begin{aligned}
y_{i j} \mid \gamma_{i 1}, \gamma_{i 2} & \stackrel{\text { ind }}{\sim} B e\left(\mu_{i j}, \sigma_{i j}\right), \\
\gamma_{i 1} & \stackrel{\text { i.i.d. }}{\sim} N\left(0, \lambda_{1}^{2}\right), \\
\gamma_{i 2} & \stackrel{\text { i.i.d. }}{\sim} N\left(0, \lambda_{2}^{2}\right),
\end{aligned}
$$

com

$$
\begin{aligned}
& \mathrm{g}\left(\mu_{i j}\right)=\beta_{11}+\beta_{21} x_{i j}+\beta_{31} t_{i}+\gamma_{i 1}, \\
& \mathrm{~g}\left(\sigma_{i j}\right)=\beta_{12}+\beta_{22} x_{i j}+\beta_{32} t_{i}+\gamma_{i 2},
\end{aligned}
$$

em que $i=1, \ldots, N, j=1, \ldots, n_{i}, \mathrm{~g}(\cdot)$ é a função de ligação logito, $\boldsymbol{\beta}_{1}=\left(\beta_{11}, \beta_{21}, \beta_{31}\right)^{\top}$ e $\boldsymbol{\beta}_{2}=\left(\beta_{12}, \beta_{22}, \beta_{32}\right)^{\top}$ são os vetores de parâmetros associados a $\mu$ e $\sigma$. Para cada cenário, 10000 repetições foram geradas a partir do modelo especificado com os seguintes valores fixos dos parâmetros: $\boldsymbol{\beta}_{1}=\boldsymbol{\beta}_{2}=(-0,15 ; 0,15 ;-0,15)^{\top}$.

Seguindo o delineamento de simulações de trabalhos com dados longitudinais, por exemplo, Park e Wu (2006), Guoyou e Zhongyi (2008) e Fu e Wang (2012), a variável explicativa $x_{i j}$ foi gerada de acordo com uma distribuição uniforme $U(0 ; 1)$ e a variável tempo $t_{i}$, com valores entre zero e um, foi gerada como $t_{i}=\left(n_{i}-1\right) / n_{i}$. Os interceptos aleatórios independentes foram gerados com distribuições normais, $\gamma_{i 1} \sim N\left(0, \lambda_{1}^{2}\right)$ e $\gamma_{i 2} \sim N\left(0, \lambda_{2}^{2}\right)$.

Consideramos todas as combinações de números de indivíduos, $N(20 ; 40 ; 60 ; 100 ; 150)$, número de observações por indivíduo, $n_{i}(3 ; 5 ; 8 ; 12)$ e desvio padrão dos interceptos aleatórios, $\lambda_{1}, \lambda_{2},(0,5 ; 1,0 ; 1.5)$. Com a combinação dos valores de $\boldsymbol{\beta}_{1}, \boldsymbol{\beta}_{2}, \lambda_{1}$ e $\lambda_{2}$ obtemos valores da média e da variância de $y_{i j}$ próximos de 0,5 . Utilizamos a função GHQ do pacote BLMM para calcular os pontos e pesos de quadratura com $Q_{1}=Q_{2}=8$ pontos de quadratura e a função nlminb para maximizar o logaritmo da função de verossimilhança (3.16).

O desempenho do processo de estimação foi avaliado por meio da raiz do erro quadrático médio (REQM) proposta por Wissel (2009) e definida como

$$
R E Q M=\left(\operatorname{traO}(\Sigma(\hat{\boldsymbol{\theta}}))+(\hat{\boldsymbol{\theta}}-\boldsymbol{\theta})^{\top}(\hat{\boldsymbol{\theta}}-\boldsymbol{\theta})\right)^{1 / 2}
$$

Valores da REQM próximos de zero indicam bom desempenho no processo de estimação.

As Tabelas 3.2, 3.3 e 3.4 apresentam os valores médios da REQM para os 10000 conjuntos de dados simulados com desvio padrão $\lambda_{1}=0,5 ; 1,0$ e 1,5, respectivamente.

Podemos notar, em todos os casos, que a REQM diminui conforme o número de observações por indivíduo $n_{i}$ aumenta, para valores fixos de número de indivíduos $N$ e de desvio padrão dos interceptos aleatórios $\left(\lambda_{1}, \lambda_{2}\right)$, indicando que o desempenho do processo 
de estimação melhora com uma maior quantidade de informação por indivíduo. Na Tabela 3.2, por exemplo, para $\lambda_{1}=0,5 ; \lambda_{2}=0,5$ e $N=150$ os valores da REQM são $(0,476 ; 0,339 ; 0,252 ; 0,210)$ para $n_{i}=3 ; 5 ; 8$ e 12 , respectivamente. A REQM também diminui conforme o número de indivíduos $N$ aumenta, para valores fixos de número de observações por indivíduo $n_{i}$ e de desvio padrão dos interceptos aleatórios $\left(\lambda_{1}, \lambda_{2}\right)$. Como exemplo, na Tabela 3.3, para $\lambda_{1}=1,0 ; \lambda_{2}=0,5$ e $n_{i}=12$, os valores da REQM são $(0,842 ; 0,561 ; 0,449 ; 0,346 ; 0,284)$ para $N=20 ; 40 ; 60 ; 100$ e 150 , respectivamente. Além disso, podemos notar que a REQM aumenta conforme o valor de $\lambda_{2}$ aumenta, para valores fixos de $\lambda_{1}$, do número de indivíduos $N$ e do número de observações por indivíduo $n_{i}$, o que indica que o desempenho do processo de estimação diminui conforme a variabilidade do intercepto aleatório em $\sigma$ aumenta. Na Tabela 3.4, por exemplo, para $\lambda_{1}=1,5 ; N=60$ e $n_{i}=3$, os valores da REQM são $(1,134 ; 1,244 ; 1,364)$ para $\lambda_{2}=0,5 ; 1,0$ e 1,5 , respectivamente. A exceção esta nos casos de $N=20, n_{i}=3, \lambda_{1}=0,5 ; 1,0$ e 1,5 e $N=20$, $n_{i}=5, \lambda_{1}=0,5$. Notamos também, de acordo com os resultados das Tabelas 3.2 a 3.4, que a REQM aumenta conforme $\lambda_{1}$ aumenta, para $N, n_{i}$ and $\lambda_{2}$ fixos. Por exemplo, para $\lambda_{2}=1,5 ; N=60$ e $n_{i}=3$, os valores da RMSE são $(1,062 ; 1,192 ; 1,364)$ para $\lambda_{1}=0,5 ; 1,0$ e 1,5 .

Em geral, a partir do estudo de simulação realizado podemos concluir que o processo de estimação tem melhores resultados quando existe uma maior quantidade de informação e quando a variabilidade dos interceptos aleatórios é pequena. 
Tabela 3.2: REQM das estimativas dos parâmetros do modelo beta normal com $\lambda_{1}=0,5$.

\begin{tabular}{ccccc}
\hline$N$ & $n_{i}$ & $\lambda_{2}=0,5$ & $\lambda_{2}=1,0$ & $\lambda_{2}=1,5$ \\
\hline \multirow{4}{*}{20} & 3 & 2,897 & 3,188 & 2,296 \\
& 5 & 1,388 & 1,499 & 1,497 \\
& 8 & 0,918 & 0,995 & 1,116 \\
& 12 & 0,706 & 0,807 & 0,950 \\
\hline \multirow{4}{*}{40} & 3 & 1,250 & 1,462 & 1,472 \\
& 5 & 0,781 & 0,860 & 0,954 \\
& 8 & 0,572 & 0,638 & 0,782 \\
& 12 & 0,435 & 0,558 & 0,745 \\
\hline \multirow{4}{*}{60} & 3 & 0,937 & 1,014 & 1,062 \\
& 5 & 0,590 & 0,663 & 0,756 \\
& 8 & 0,430 & 0,518 & 0,658 \\
& 12 & 0,347 & 0,443 & 0,656 \\
\hline \multirow{4}{*}{100} & 3 & 0,638 & 0,688 & 0,727 \\
& 5 & 0,432 & 0,459 & 0,570 \\
& 8 & 0,315 & 0,387 & 0,540 \\
& 12 & 0,257 & 0,356 & 0,537 \\
\hline \multirow{4}{*}{150} & 3 & 0,476 & 0,528 & 0,566 \\
& 5 & 0,339 & 0,368 & 0,466 \\
& 8 & 0,252 & 0,314 & 0,478 \\
& 12 & 0,210 & 0,295 & 0,407 \\
\hline
\end{tabular}

Tabela 3.3: REQM das estimativas dos parâmetros do modelo beta normal com $\lambda_{1}=1,0$.

\begin{tabular}{ccccc}
\hline$N$ & $n_{i}$ & $\lambda_{2}=0,5$ & $\lambda_{2}=1,0$ & $\lambda_{2}=1,5$ \\
\hline \multirow{4}{*}{20} & 3 & 3,428 & 3,401 & 3,287 \\
& 5 & 1,601 & 1,683 & 1,773 \\
& 8 & 1,075 & 1,135 & 1,312 \\
& 12 & 0,842 & 0,978 & 1,136 \\
\hline \multirow{4}{*}{40} & 3 & 1,486 & 1,594 & 1,652 \\
& 5 & 0,906 & 0,953 & 1,083 \\
& 8 & 0,670 & 0,760 & 0,969 \\
& 12 & 0,561 & 0,677 & 0,929 \\
\hline \multirow{6}{*}{60} & 3 & 0,995 & 1,127 & 1,192 \\
& 5 & 0,679 & 0,770 & 0,891 \\
& 8 & 0,514 & 0,635 & 0,816 \\
& 12 & 0,449 & 0,572 & 0,814 \\
\hline \multirow{4}{*}{100} & 3 & 0,683 & 0,767 & 0,859 \\
& 5 & 0,484 & 0,561 & 0,708 \\
& 8 & 0,391 & 0,486 & 0,685 \\
& 12 & 0,346 & 0,467 & 0,677 \\
\hline \multirow{4}{*}{150} & 3 & 0,531 & 0,574 & 0,697 \\
& 5 & 0,375 & 0,438 & 0,598 \\
& 8 & 0,314 & 0,411 & 0,593 \\
& 12 & 0,284 & 0,390 & 0,540 \\
\hline
\end{tabular}


Tabela 3.4: REQM das estimativas dos parâmetros do modelo beta normal com $\lambda_{1}=1,5$.

\begin{tabular}{ccccc}
\hline$N$ & $n_{i}$ & $\lambda_{2}=0,5$ & $\lambda_{2}=1,0$ & $\lambda_{2}=1,5$ \\
\hline \multirow{4}{*}{20} & 3 & 3,577 & 3,619 & 3,506 \\
& 5 & 1,811 & 1,822 & 1,868 \\
& 8 & 1,279 & 1,369 & 1,543 \\
& 12 & 1,075 & 1,191 & 1,458 \\
\hline \multirow{4}{*}{40} & 3 & 1,645 & 1,737 & 1,789 \\
& 5 & 1,031 & 1,157 & 1,301 \\
& 8 & 0,857 & 1,000 & 1,189 \\
& 12 & 0,771 & 0,928 & 1,181 \\
\hline \multirow{4}{*}{60} & 3 & 1,134 & 1,244 & 1,364 \\
& 5 & 0,816 & 0,903 & 1,092 \\
& 8 & 0,693 & 0,816 & 1,061 \\
& 12 & 0,646 & 0,805 & 1,050 \\
\hline \multirow{4}{*}{100} & 3 & 0,777 & 0,859 & 1,005 \\
& 5 & 0,593 & 0,692 & 0,909 \\
& 8 & 0,556 & 0,677 & 0,901 \\
& 12 & 0,553 & 0,675 & 0,895 \\
\hline \multirow{4}{*}{150} & 3 & 0,599 & 0,682 & 0,963 \\
& 5 & 0,491 & 0,641 & 0,861 \\
& 8 & 0,490 & 0,590 & 0,822 \\
& 12 & 0,454 & 0,573 & 0,792 \\
\hline
\end{tabular}

\subsection{Conclusões}

Neste capítulo abordamos o problema de modelar a relação entre uma variável resposta com uma distribuição beta medida ao longo do tempo, e um conjunto de variáveis explicativas. Vários aspectos foram levados em conta ao serem propostos os modelos de regressão beta com interceptos aleatórios em que a variável resposta é parametrizada em termos da média e da dispersão.

A flexibilidade da formulação proposta permite explicar de maneira mais clara as relações que descrevem a heterogeneidade de médias entre indivíduos e a dispersão entre observações repetidas num indivíduo usando preditores lineares e funções de ligação adequadas. Propomos um método de estimação dos parâmetros desses modelos e uma metodologia para obter as predições dos interceptos aleatórios. Para aproximar a função de verossimilhança foi utilizado o método de quadratura de Gauss-Hermite. A predição dos interceptos aleatórios foi realizada considerando a metodologia do melhor preditor de Bayes empírico. Vale a pena ressaltar que a metodologia proposta permite obter os componentes de variância dos interceptos aleatórios em conjunto com as estimativas de máxima verossimilhança dos efeitos fixos, sem a necessidade da especificação de distribuições a priori e hiperparâmetros.

O estudo de simulação mostrou que o aumento no número de indivíduos pode melhorar o desempenho do processo de estimação. Além disso, os resultados mostram que o desempenho aumenta conforme a variabilidade dos interceptos aleatórios diminui. 


\section{Capítulo 4}

\section{Modelo de regressão beta com intercepto aleatório não normal}

A suposição de normalidade dos efeitos aleatórios é usual nos modelos lineares mistos pela conveniência matemática e computacional uma vez que a verossimilhança marginal pode ser expressa em forma fechada e a inferência estatística pode ser realizada usando técnicas de otimização convencionais e disponíveis em pacotes computacionais amplamente conhecidos. Segundo Pinheiro et al. (2001) e Zhang e Davidian (2001) apesar destes modelos oferecerem flexibilidade na modelagem da correlação em cada indivíduo a suposição de normalidade é muito restrita e sofre de falta de robustez contra observações discrepantes e, portanto, estes modelos não podem fornecer estimativas exatas da variação entre indivíduos. Embora alguns estudos sugerem que a inferência sobre os efeitos fixos pode ser robusta à não normalidade dos efeitos aleatórios (Verbeke e Lesaffre, 1997), há também resultados de inconsistências nas estimativas de efeitos fixos e aleatórios sob a especificação incorreta da distribuição dos efeitos aleatórios (McCulloch e Neuhaus, 2011). Assim, é de interesse relaxar a suposição de normalidade na distribuição dos efeitos aleatórios. Alguns exemplos de trabalhos com este interesse incluem o trabalho de Lin e Lee (2008) sobre modelo linear misto com efeitos aleatórios normais assimétricos para dados longitudinais, o artigo de Zhang et al. (2008) que trata de um modelo linear misto com distribuição log-gama na inclinação aleatória e o trabalho de Fabio et al. (2012) que aborda um modelo misto Poisson com distribuição log-gama generalizada para os efeitos aleatórios.

O modelo considerado neste capítulo estende o modelo proposto no Capítulo 3, no sentido que aqui consideramos interceptos aleatórios com distribuição não normal.

Na Seção 4.1 apresentamos o modelo proposto. Na Seção 4.2 é desenvolvido um método de estimação dos parâmetros do modelo com a abordagem proposta por Liu e Yu (2008). Um estudo de simulação que tem por objetivo avaliar o desempenho do processo de estimação dos parâmetros é apresentado na Seção 4.4. Finalmente, na Seção 4.5 são apresentadas as 
conclusões do capítulo.

\subsection{Modelo proposto}

Nesta seção apresentamos o modelo de regressão beta com intercepto aleatório não normal e mostramos três modelos que podem ser considerados para análise de dados na forma de taxas, porcentagens ou proporções medidas em escala contínua ao longo do tempo.

Seja $y_{i j}$ o valor da variável resposta para o $i$-ésimo indivíduo no tempo $t_{i j}, i=1, \ldots, N$, $j=1, \ldots, n_{i}$. No modelo beta com intercepto aleatório não normal assumimos que, condicional aos efeitos aleatórios $\gamma_{i 1}$ e $\gamma_{i 2}$, as respostas $y_{i 1}, y_{i 2}, \ldots, y_{i n_{i}}$ são independentes e cada uma segue uma distribuição beta com função densidade dada por (2.8) e os efeitos aleatórios $\gamma_{i 1}$ e $\gamma_{i 2}$ são independentes e identicamente distribuídos com função densidade $f\left(\gamma_{i 1} ; \boldsymbol{\lambda}_{1}\right)$ e $f\left(\gamma_{i 2} ; \boldsymbol{\lambda}_{2}\right)$, respectivamente. A representação do modelo é dada por

$$
\begin{aligned}
y_{i j} \mid \gamma_{i 1}, \gamma_{i 2} & \stackrel{\text { ind }}{\sim} B e\left(\mu_{i j}, \sigma_{i j}\right), \\
\gamma_{i 1} & \stackrel{\text { i.i.d }}{\sim} f\left(\gamma_{i 1} ; \boldsymbol{\lambda}_{1}\right), \\
\gamma_{i 2} & \stackrel{\text { i.i.j. }}{\sim} f\left(\gamma_{i 2} ; \boldsymbol{\lambda}_{2}\right),
\end{aligned}
$$

e pelos componentes sistemáticos

$$
\begin{aligned}
& g_{1}\left(\mu_{i j}\right)=\eta_{i j 1}=\boldsymbol{x}_{i j 1}^{\top} \boldsymbol{\beta}_{1}+\gamma_{i 1}, \\
& g_{2}\left(\sigma_{i j}\right)=\eta_{i j 2}=\boldsymbol{x}_{i j 2}^{\top} \boldsymbol{\beta}_{2}+\gamma_{i 2},
\end{aligned}
$$

para $i=1,2, \ldots, N$ e $j=1,2, \ldots, n_{i}$, em que $\boldsymbol{x}_{i j 1}=\left(x_{i j 11}, x_{i j 21}, \ldots, x_{i j p_{1} 1}\right)^{\top}$ e $\boldsymbol{x}_{i j 2}=$ $\left(x_{i j 12}, x_{i j 22}, \ldots, x_{i j p_{2} 2}\right)^{\top}$ são vetores associados aos efeitos fixos de dimensão $p_{1} \times 1$ e $p_{2} \times 1$, respectivamente; $\boldsymbol{\beta}_{1}=\left(\beta_{11}, \beta_{21}, \ldots, \beta_{p_{1} 1}\right)^{\top}$ e $\boldsymbol{\beta}_{2}=\left(\beta_{12}, \beta_{22}, \ldots, \beta_{p_{2} 2}\right)^{\top}$ são vetores de parâmetros desconhecidos a serem estimados de dimensões $p_{1} \times 1$ e $p_{2} \times 1$, respectivamente e $\gamma_{i 1}$ e $\gamma_{i 2}$ são os interceptos aleatórios associados ao $i$-ésimo indivíduo. As funções de ligação $g_{1}:(0,1) \rightarrow \mathbb{R}$ e $g_{2}:(0,1) \rightarrow \mathbb{R}$ são funções conhecidas, estritamente monótonas a duas vezes diferenciáveis. Alguns exemplos dessas funções foram apresentados na Seção 3.1.

As funções densidade de probabilidade $f\left(\gamma_{i 1} ; \boldsymbol{\lambda}_{1}\right)$ e $f\left(\gamma_{i 2} ; \boldsymbol{\lambda}_{2}\right)$ denotam as distribuições dos interceptos aleatórios com média zero e vetor de parâmetros $\boldsymbol{\lambda}_{1}$ e $\boldsymbol{\lambda}_{2}$, respectivamente. A seguir serão apresentados três modelos de regressão beta com efeitos aleatórios não normais que seguem a estrutura do modelo dado em (4.1) e em (4.2). 


\subsubsection{Modelo beta log-gama}

Supondo que os $y_{i j}$ seguem uma distribuição beta com função densidade de probabilidade dada por (2.8) e os efeitos aleatórios $\gamma_{i 1}$ e $\gamma_{i 2}$ seguem uma distribuição log-gama com função densidade de probabilidade dada por (2.9) tem-se que o modelo beta log-gama é representado por

$$
\begin{aligned}
y_{i j} \mid \gamma_{i 1}, \gamma_{i 2} & \stackrel{\text { ind }}{\sim} \operatorname{Be}\left(\mu_{i j}, \sigma_{i j}\right), \\
\gamma_{i 1} & \stackrel{\text { i.i.d }}{\sim} \operatorname{LG}\left(\lambda_{1}\right), \\
\gamma_{i 2} & \stackrel{\text { i.i.d }}{\sim} \operatorname{LG}\left(\lambda_{2}\right),
\end{aligned}
$$

e pelos componentes sistemáticos (4.2). Neste modelo o vetor de parâmetros é dado por $\boldsymbol{\theta}=\left(\boldsymbol{\beta}_{1}^{\top}, \boldsymbol{\beta}_{2}^{\top}, \lambda_{1}, \lambda_{2}\right)^{\top}$, com espaço paramétrico associado dado por $\boldsymbol{\Theta}=\left\{\boldsymbol{\theta} \in \mathbb{R}^{p} \mid \boldsymbol{\beta}_{1} \in \mathbb{R}^{p_{1}}\right.$, $\left.\boldsymbol{\beta}_{2} \in \mathbb{R}^{p_{2}}, \lambda_{1} \in \mathbb{R}^{+}, \lambda_{2} \in \mathbb{R}^{+}\right\}$, em que $p=p_{1}+p_{2}+2$.

\subsubsection{Modelo beta $t$-Student}

Considerando-se a distribuição beta com função densidade de probabilidade dada por (2.8) para $y_{i j}$ e a distribuição $t$-Student para os efeitos aleatórios $\gamma_{i 1}$ e $\gamma_{i 2}$ dada por (2.10), o modelo beta $t$-Student é dado por

$$
\begin{gathered}
y_{i j} \mid \gamma_{i 1}, \gamma_{i 2} \stackrel{\text { ind }}{\sim} B e\left(\mu_{i j}, \sigma_{i j}\right), \\
\gamma_{i 1} \stackrel{\text { i.i.d }}{\sim} t_{\nu_{1}}, \\
\gamma_{i 2} \stackrel{\text { i.i.d. }}{\sim} t_{\nu_{2}},
\end{gathered}
$$

e pelos componentes sistemáticos (4.2). O vetor de parâmetros associado a este modelo é dado por $\boldsymbol{\theta}=\left(\boldsymbol{\beta}_{1}^{\top}, \boldsymbol{\beta}_{2}^{\top}\right)^{\top}$, com espaço paramétrico $\boldsymbol{\Theta}=\left\{\boldsymbol{\theta} \in \mathbb{R}^{p} \mid \boldsymbol{\beta}_{1} \in \mathbb{R}^{p_{1}}, \boldsymbol{\beta}_{2} \in \mathbb{R}^{p_{2}}\right\}$, em que $p=p_{1}+p_{2}$. Dado que estamos assumindo fixos e conhecidos os graus de liberdade $\nu_{1}$ e $\nu_{2}$ podemos particularizar o modelo beta $t$-Student usando valores de um nos graus de liberdade de ambas as distribuições $t$-Student $\left(\nu_{1}=1, \nu_{2}=1\right)$ e denominá-lo de modelo beta Cauchy.

\subsubsection{Modelo beta exponencial potência}

Supondo que $y_{i j}$ segue uma distribuição beta com função densidade de probabilidade dada por (2.8) e os interceptos aleatórios $\gamma_{i 1}$ e $\gamma_{i 2}$ seguem uma distribuição exponencial potência dada por (2.11) tem-se que a representação do modelo beta exponencial potência 
é dada por

$$
\begin{aligned}
y_{i j} \mid \gamma_{i 1}, \gamma_{i 2} & \stackrel{\text { ind }}{\sim} \operatorname{Be}\left(\mu_{i j}, \sigma_{i j}\right), \\
\gamma_{i 1} & \stackrel{\text { i.i.d }}{\sim} \operatorname{EP}\left(0, \lambda_{1}, \nu_{1}\right), \\
\gamma_{i 2} & \stackrel{\text { i.i.d }}{\sim} \operatorname{EP}\left(0, \lambda_{2}, \nu_{2}\right),
\end{aligned}
$$

e pelos componentes sistemáticos (4.2). Assumindo fixos e conhecidos $\nu_{1}$ e $\nu_{2}$, o vetor de parâmetros deste modelo é dado por $\boldsymbol{\theta}=\left(\boldsymbol{\beta}_{1}^{\top}, \boldsymbol{\beta}_{2}^{\top}, \lambda_{1}, \lambda_{2}\right)^{\top}$, em que o espaço paramétrico é definido como $\boldsymbol{\Theta}=\left\{\boldsymbol{\theta} \in \mathbb{R}^{p} \mid \boldsymbol{\beta}_{1} \in \mathbb{R}^{p_{1}}, \boldsymbol{\beta}_{2} \in \mathbb{R}^{p_{2}}, \lambda_{1} \in \mathbb{R}^{+}, \lambda_{2} \in \mathbb{R}^{+}\right\}$, em que $p=p_{1}+p_{2}+2$. Se para ambos os efeitos aleatórios se considera o parâmetro de curtose fixo e igual a um $\left(\nu_{1}=1, \nu_{2}=1\right)$ o modelo é chamado de modelo beta Laplace.

As expressões para a média, variância, covariância e correlação da distribuição marginal de $y_{i j}$ para os modelos com intercepto aleatório não normal são as mesmas que as apresentadas nas Seções 3.1.1, 3.1.2 e 3.1.3, já que estas não dependem da distribuição dos efeitos aleatórios. As expressões da média e variância da distribuição marginal de $y_{i j}$ serão utilizadas no Capítulo 6

\subsection{Estimação}

De forma similar ao modelo de regressão beta com intercepto aleatório normal a inferência estatística para o modelo de regressão beta com efeitos aleatórios não normais é baseada no método de máxima verossimilhança como se descreve a seguir.

Considerando o modelo determinado por (4.1) e (4.2) e sendo $\boldsymbol{y}_{i}=\left(y_{i 1}, y_{i 2}, \ldots, y_{i n_{i}}\right)^{\top}$ o vetor de observações no indivíduo $i$, a função densidade de probabilidade conjunta de $\left(\boldsymbol{y}_{i}, \gamma_{i 1}, \gamma_{i 2}\right)$ é dada por

$$
f\left(\boldsymbol{y}_{i}, \gamma_{i 1}, \gamma_{i 2} ; \boldsymbol{\theta}\right)=\prod_{j=1}^{n_{i}} f\left(y_{i j} \mid \gamma_{i 1}, \gamma_{i 2} ; \boldsymbol{\beta}_{1}, \boldsymbol{\beta}_{2}\right) \cdot f\left(\gamma_{i 1} ; \boldsymbol{\lambda}_{1}\right) f\left(\gamma_{i 2} ; \boldsymbol{\lambda}_{2}\right)
$$

e a função de verossimilhança para $\boldsymbol{\theta}$ baseada em $\boldsymbol{y}_{i}$ é dada por

$$
L_{i}(\boldsymbol{\theta})=\int_{\mathbb{R}^{2}} \prod_{j=1}^{n_{i}} f\left(y_{i j} \mid \gamma_{i 1}, \gamma_{i 2} ; \boldsymbol{\beta}_{1}, \boldsymbol{\beta}_{2}\right) \cdot f\left(\gamma_{i 1} ; \boldsymbol{\lambda}_{1}\right) f\left(\gamma_{i 2} ; \boldsymbol{\lambda}_{2}\right) d \gamma_{i 1} d \gamma_{i 2},
$$

em que $f\left(y_{i j} \mid \gamma_{i 1}, \gamma_{i 2} ; \boldsymbol{\beta}_{1}, \boldsymbol{\beta}_{2}\right)$ é a função densidade de probabilidade condicional de $y_{i j}$ dados $\gamma_{i 1}, \gamma_{i 2}$ e $f\left(\gamma_{i 1} ; \boldsymbol{\lambda}_{1}\right)$ e $f\left(\gamma_{i 2} ; \boldsymbol{\lambda}_{2}\right)$ são as respectivas funções densidades de probabilidades de $\gamma_{i 1}$ e $\gamma_{i 2}$. 
Como já foi discutido no Capítulo 3 a função de verossimilhança (4.6) não tem expresão analítica ou de forma fechada e precisa-se usar algum método numérico para aproximar essa função e posteriormente utilizar métodos de otimização para a obtenção dos pontos de maximização.

Uma abordagem alternativa para obter as estimativas de máxima verossimilhança aproximada é usar o método de reformulação da verossimilhança para modelos de efeitos aleatórios não normais proposto por Liu e Yu (2008). Essa abordagem foi usada em modelos com efeitos aleatórios log-gama e de mistura de duas normais.

O método consiste em multiplicar e dividir o integrando de $L_{i}(\boldsymbol{\theta})$ por duas densidades normais padrão, $\phi\left(\gamma_{i 1}\right)$ e $\phi\left(\gamma_{i 2}\right)$, como segue

$$
L_{i}(\boldsymbol{\theta})=\int_{\mathbb{R}^{2}} \prod_{j=1}^{n_{i}} f\left(y_{i j} \mid \gamma_{i 1}, \gamma_{i 2} ; \boldsymbol{\beta}_{1}, \boldsymbol{\beta}_{2}\right) \cdot \frac{f\left(\gamma_{i 1} ; \boldsymbol{\lambda}_{1}\right)}{\phi\left(\gamma_{i 1}\right)} \frac{f\left(\gamma_{i 2} ; \boldsymbol{\lambda}_{2}\right)}{\phi\left(\gamma_{i 2}\right)} \phi\left(\gamma_{i 1}\right) \phi\left(\gamma_{i 2}\right) d \gamma_{i 1} d \gamma_{i 2} .
$$

Uma vez reformulada $L_{i}(\boldsymbol{\theta})$ podemos definir a função de verossimilhança para $\boldsymbol{\theta}$ baseada em $\boldsymbol{y}=\left(\boldsymbol{y}_{1}, \ldots, \boldsymbol{y}_{N}\right)^{\top}$ como

$$
L(\boldsymbol{\theta})=\prod_{i=1}^{N} \int_{\mathbb{R}^{2}} \prod_{j=1}^{n_{i}} f\left(y_{i j} \mid \gamma_{i 1}, \gamma_{i 2} ; \boldsymbol{\beta}_{1}, \boldsymbol{\beta}_{2}\right) \cdot \frac{f\left(\gamma_{i 1} ; \boldsymbol{\lambda}_{1}\right)}{\phi\left(\gamma_{i 1}\right)} \frac{f\left(\gamma_{i 2} ; \boldsymbol{\lambda}_{2}\right)}{\phi\left(\gamma_{i 2}\right)} \phi\left(\gamma_{i 1}\right) \phi\left(\gamma_{i 2}\right) d \gamma_{i 1} d \gamma_{i 2},
$$

de modo que o logaritmo da função de verossimilhança pode ser escrito como

$$
\ell(\boldsymbol{\theta})=\sum_{i=1}^{N} \log \left(\int_{\mathbb{R}^{2}} \prod_{j=1}^{n_{i}} f\left(y_{i j} \mid \gamma_{i 1}, \gamma_{i 2} ; \boldsymbol{\beta}_{1}, \boldsymbol{\beta}_{2}\right) \cdot \frac{f\left(\gamma_{i 1} ; \boldsymbol{\lambda}_{1}\right)}{\phi\left(\gamma_{i 1}\right)} \frac{f\left(\gamma_{i 2} ; \boldsymbol{\lambda}_{2}\right)}{\phi\left(\gamma_{i 2}\right)} \phi\left(\gamma_{i 1}\right) \phi\left(\gamma_{i 2}\right) d \gamma_{i 1} d \gamma_{i 2}\right) .
$$

Assim, podemos usar a quadratura de Gauss-Hermite multidimensional dada em (2.5) para aproximar $\ell(\boldsymbol{\theta})$ como segue

$$
\ell(\boldsymbol{\theta}) \cong \sum_{i=1}^{N} \log \left(\sum_{k_{1}=1}^{Q_{1}} \sum_{k_{2}=1}^{Q_{2}} \prod_{j=1}^{n_{i}} f\left(y_{i j} \mid z_{k_{1}}, z_{k_{2}} ; \boldsymbol{\beta}_{1}, \boldsymbol{\beta}_{2}\right) \cdot \frac{f\left(z_{k_{1}} ; \boldsymbol{\lambda}_{1}\right)}{\phi\left(z_{k_{1}}\right)} \frac{f\left(z_{k_{2}} ; \boldsymbol{\lambda}_{2}\right)}{\phi\left(z_{k_{2}}\right)} w_{k_{1}} w_{k_{2}}\right) .
$$

Para estimar o vetor de parâmetros dos modelos de regressão beta com intercepto aleatório log-gama, $t$-Student e exponencial potência, podemos usar a função blmmML do pacote BLMM. Os pontos e pesos da quadratura de Gauss-Hermite multidimensional podem ser calculados com a função GHQ. Detalhes das funções são apresentados no Capítulo 8.

\subsection{Predição dos efeitos aleatórios}

Para os modelos de regressão beta com interceptos aleatórios não normais propomos usar o método do preditor de Bayes empírico para obter o melhor preditor dos interceptos 
aleatórios. Para resolver as integrais envolvidas na predição usamos a aproximação de Liu e Yu (2008), de modo que a predição para $\gamma_{i 1}$ e $\gamma_{i 2}$ é dada por

$$
\begin{aligned}
\widetilde{\gamma}_{i 1}= & \mathrm{E}\left[\gamma_{i 1} \mid y_{i j} ; \hat{\boldsymbol{\theta}}\right] \\
= & \frac{\int_{\mathbb{R}^{2}} \gamma_{i 1} \prod_{j=1}^{n i} f\left(y_{i j} \mid \gamma_{i 1}, \gamma_{i 2} ; \hat{\boldsymbol{\beta}}_{1}, \hat{\boldsymbol{\beta}}_{2}\right) \frac{f\left(\gamma_{i 1} ; \hat{\boldsymbol{\lambda}}_{1}\right)}{\phi\left(\gamma_{i 1}\right)} \frac{f\left(\gamma_{i 2} ; \hat{\boldsymbol{\lambda}}_{2}\right)}{\phi\left(\gamma_{i 2}\right)} \phi\left(\gamma_{i 1}\right) \phi\left(\gamma_{i 2}\right) d \gamma_{i 1} d \gamma_{i 2}}{\int_{\mathbb{R}^{2}} \prod_{j=1}^{n i} f\left(y_{i j} \mid \gamma_{i 1}, \gamma_{i 2} ; \hat{\boldsymbol{\beta}}_{1}, \hat{\boldsymbol{\beta}}_{2}\right) \frac{f\left(\gamma_{i 1} ; \hat{\boldsymbol{\lambda}}_{1}\right)}{\phi\left(\gamma_{i 1}\right)} \frac{f\left(\gamma_{i 2} ; \hat{\boldsymbol{\lambda}}_{2}\right)}{\phi\left(\gamma_{i 2}\right)} \phi\left(\gamma_{i 1}\right) \phi\left(\gamma_{i 2}\right) d \gamma_{i 1} d \gamma_{i 2}}, \\
\widetilde{\gamma}_{i 2}=\mathrm{E}\left[\gamma_{i 2} \mid y_{i j} ; \hat{\boldsymbol{\theta}}\right] & \frac{\int_{\mathbb{R}^{2}} \gamma_{i 2} \prod_{j=1}^{n i} f\left(y_{i j} \mid \gamma_{i 1}, \gamma_{i 2} ; \hat{\boldsymbol{\beta}}_{1}, \hat{\boldsymbol{\beta}}_{2}\right) \frac{f\left(\gamma_{i 1} ; \hat{\boldsymbol{\lambda}}_{1}\right)}{\phi\left(\gamma_{i 1}\right)} \frac{f\left(\gamma_{i 2} ; \hat{\boldsymbol{\lambda}}_{2}\right)}{\phi\left(\gamma_{i 2}\right)} \phi\left(\gamma_{i 1}\right) \phi\left(\gamma_{i 2}\right) d \gamma_{i 1} d \gamma_{i 2}}{\int_{\mathbb{R}^{2}} \prod_{j=1}^{n i} f\left(y_{i j} \mid \gamma_{i 1}, \gamma_{i 2} ; \hat{\boldsymbol{\beta}}_{1}, \hat{\boldsymbol{\beta}}_{2}\right) \frac{f\left(\gamma_{i 1} ; \hat{\boldsymbol{\lambda}}_{1}\right)}{\phi\left(\gamma_{i 1}\right)} \frac{f\left(\gamma_{i 2} ; \hat{\boldsymbol{\lambda}}_{2}\right)}{\phi\left(\gamma_{i 2}\right)} \phi\left(\gamma_{i 1}\right) \phi\left(\gamma_{i 2}\right) d \gamma_{i 1} d \gamma_{i 2}},
\end{aligned}
$$

em que $i=1,2, \ldots, N$ e $j=1,2, \ldots, n_{i}$. As integrais no numerador e denominador de (4.9) e (4.10) são resolvidas por meio da quadratura de Gauss-Hermite, da mesma forma que a integral (4.7). A predição dos efeitos aleatórios não normais é implementada na função re.prediction do pacote BLMM explicada em detalhes no Capítulo 8.

\subsection{Estudo de simulação}

Nesta seção apresentamos resultados de um estudo de simulação que considera vários cenários para o modelo de regressão beta com intercepto aleatório, a fim de avaliar o desempenho do processo de estimação quando a distribuição dos efeitos aleatórios segue uma distribuição não normal.

Analisamos três diferentes cenários descritos a seguir para o modelo de regressão beta com intercepto aleatório não normal. No primeiro cenário consideramos o modelo de regressão beta com intercepto aleatório log-gama. O modelo de regressão beta com intercepto aleatório t-Student é considerando no segundo cenário. No último cenário consideramos o modelo de regressão beta exponencial potência. As variáveis consideradas na estrutura de regressão dos modelos foram $x_{i j}$ e $t_{i}$, geradas de acordo com uma distribuição uniforme $U(0 ; 1)$ e de acordo com a expressão $t_{i}=\left(n_{i}-1\right) / n_{i}$, respectivamente. Para cada cenário 10000 réplicas foram obtidas e para cada um dos vetores de parâmetros $\boldsymbol{\beta}_{1}=\left(\beta_{11}, \beta_{21}, \beta_{31}\right)^{\top}$ e $\boldsymbol{\beta}_{2}=\left(\beta_{12}, \beta_{22}, \beta_{32}\right)^{\top}$ foram considerados os seguintes valores fixos: $\boldsymbol{\beta}_{1}=\boldsymbol{\beta}_{2}=(-0,15 ; 0,15 ;-0,15)^{\top}$.

Consideramos $N=20 ; 40 ; 60 ; 100$ e 150 indivíduos e $n_{i}=3 ; 5 ; 8$ e 12 observações por indivíduo. A função logito foi escolhida para os parâmetros $\mu$ e $\sigma$. Utilizamos a função blmmML do pacote BLMM para obter as estimativas de máxima verossimilhança com $Q_{1}=Q_{2}=8$ 
pontos de quadratura.

O desempenho do processo de estimação é avaliado por meio da raiz do erro quadrático médio (REQM) definida em (3.22).

\subsubsection{Cenário 1: Modelo beta log-gama}

Neste cenário o modelo de regressão beta com intercepto aleatório log-gama apresenta a estrutura dada em (4.3) com

$$
\begin{aligned}
& g\left(\mu_{i j}\right)=\beta_{11}+\beta_{21} x_{i j}+\beta_{31} t_{i}+\gamma_{i 1} \\
& g\left(\sigma_{i j}\right)=\beta_{12}+\beta_{22} x_{i j}+\beta_{32} t_{i}+\gamma_{i 2}
\end{aligned}
$$

em que $i=1,2, \ldots, N$ e $j=1,2, \ldots, n_{i}$. Consideramos três valores diferentes para o parâmetro da distribuição log-gamma, $\lambda_{1}=\lambda_{2}=0,22 ; 0,7$ e 1,22, de modo que a variância da distribuição dos interceptos aleatórios asume valores de 0,25; 1, 0 e 2, 25, para cada combinação do número de indivíduos $N$ e número de observações por indivíduo $n_{i}$.

Os resultados são apresentados nas Tabelas 4.1 a 4.3. Destas tabelas pode-se notar que a REQM aumenta quando $\lambda_{2}$ aumenta, dados os valores de $\lambda_{1}, N$ e $n_{i}$. Por exemplo, quando $\lambda_{1}=0,22 ; N=20$ e $n_{i}=8$ a REQM é 0,$906 ; 0,912$ e 1,063 para valores de $\lambda_{2}$ de 0,22;0,70 e 1,22, respectivamente. Vale a pena destacar que o aumento no valor de $\lambda_{1}$ resulta num maior valor da REQM mas com maior efeito do que o aumento no valor de $\lambda_{2}$, por exemplo, quando $\lambda_{2}=0,22 ; N=20$ e $n_{i}=8$ a REQM é 0,906; 1,046 e 1,251 para valores de $\lambda_{1}$ de 0,$22 ;, 0,70$ e 1,22, respectivamente. Um aumento do valor de $\lambda_{1}$ e $\lambda_{2}$ indica maior assimetria na distribuição do efeito aleatório associado com a média e a dispersão, respectivamente. Também podemos destacar que em todos os casos observados quando o número de observações por indivíduo e o número de individuos aumenta, obtemos os resultados esperados, ou seja, a REQM decresce quando fixamos $\lambda_{1}$ e $\lambda_{2}$.

\subsubsection{Cenário 2: Modelo beta $t$-Student}

Neste cenário admitimos que o modelo de regressão beta com intercepto aleatório $t$ Student apresenta a estrutura (4.4) com

$$
\begin{aligned}
& g\left(\mu_{i j}\right)=\beta_{11}+\beta_{21} x_{i j}+\beta_{31} t_{i}+\gamma_{i 1}, \\
& g\left(\sigma_{i j}\right)=\beta_{12}+\beta_{22} x_{i j}+\beta_{32} t_{i}+\gamma_{i 2},
\end{aligned}
$$

para $i=1,2, \ldots, N$ e $j=1,2, \ldots, n_{i}$. Consideramos os graus de liberdade, $\nu_{1}$ e $\nu_{2}$, fixos nos valores de 3 e 4 . Com esses valores a variância da distribuição $t$-Student é dada por 3 e 2 , respectivamente. 
Tabela 4.1: REQM das estimativas dos parâmetros do modelo beta log-gama com $\lambda_{1}=0,22$.

\begin{tabular}{|c|c|c|c|c|}
\hline$N$ & $n_{i}$ & $\lambda_{2}=0,22$ & $\lambda_{2}=0,70$ & $\lambda_{2}=1,22$ \\
\hline \multirow{4}{*}{20} & 3 & 2,402 & 2,436 & 2,530 \\
\hline & 5 & 1,026 & 1,120 & 1,397 \\
\hline & 8 & 0,906 & 0,912 & 1,063 \\
\hline & 12 & 0,718 & 0,744 & 0,898 \\
\hline \multirow{4}{*}{40} & 3 & 1,202 & 1,266 & 1,347 \\
\hline & 5 & 0,734 & 0,762 & 0,864 \\
\hline & 8 & 0,559 & 0,590 & 0,690 \\
\hline & 12 & 0,476 & 0,521 & 0,628 \\
\hline \multirow{4}{*}{60} & 3 & 0,853 & 0,921 & 1,003 \\
\hline & 5 & 0,588 & 0,607 & 0,692 \\
\hline & 8 & 0,447 & 0,464 & 0,567 \\
\hline & 12 & 0,393 & 0,426 & 0,534 \\
\hline \multirow{4}{*}{100} & 3 & 0,619 & 0,629 & 0,736 \\
\hline & 5 & 0,411 & 0,442 & 0,534 \\
\hline & 8 & 0,331 & 0,367 & 0,457 \\
\hline & 12 & 0,298 & 0,353 & 0,457 \\
\hline \multirow{4}{*}{150} & 3 & 0,483 & 0,487 & 0,603 \\
\hline & 5 & 0,324 & 0,343 & 0,462 \\
\hline & 8 & 0,256 & 0,308 & 0,408 \\
\hline & 12 & 0,242 & 0,307 & 0,396 \\
\hline
\end{tabular}

Tabela 4.2: $R E Q M$ das estimativas dos parâmetros do modelo beta log-gama com $\lambda_{1}=0,70$.

\begin{tabular}{ccccc}
\hline$N$ & $n_{i}$ & $\lambda_{2}=0,22$ & $\lambda_{2}=0,70$ & $\lambda_{2}=1,22$ \\
\hline \multirow{4}{*}{20} & 3 & 2,112 & 2,595 & 2,737 \\
& 5 & 1,450 & 1,450 & 1,519 \\
& 8 & 1,046 & 1,072 & 1,206 \\
& 12 & 0,893 & 0,929 & 1,088 \\
\hline \multirow{4}{*}{40} & 3 & 1,321 & 1,349 & 1,364 \\
& 5 & 0,848 & 0,874 & 0,985 \\
& 8 & 0,635 & 0,695 & 0,836 \\
& 12 & 0,572 & 0,644 & 0,797 \\
\hline \multirow{6}{*}{60} & 3 & 0,953 & 1,000 & 1,075 \\
& 5 & 0,641 & 0,680 & 0,784 \\
& 8 & 0,507 & 0,570 & 0,679 \\
& 12 & 0,456 & 0,546 & 0,664 \\
\hline \multirow{4}{*}{100} & 3 & 0,654 & 0,670 & 0,800 \\
& 5 & 0,459 & 0,494 & 0,631 \\
& 8 & 0,384 & 0,437 & 0,551 \\
& 12 & 0,357 & 0,435 & 0,546 \\
\hline \multirow{4}{*}{150} & 3 & 0,511 & 0,527 & 0,633 \\
& 5 & 0,367 & 0,380 & 0,515 \\
& 8 & 0,307 & 0,352 & 0,482 \\
& 12 & 0,292 & 0,340 & 0,481 \\
\hline
\end{tabular}


Tabela 4.3: REQM das estimativas dos parâmetros do modelo beta log-gama com $\lambda_{1}=1,22$.

\begin{tabular}{ccccc}
\hline$N$ & $n_{i}$ & $\lambda_{2}=0,22$ & $\lambda_{2}=0,70$ & $\lambda_{2}=1,22$ \\
\hline \multirow{4}{*}{20} & 3 & 2,621 & 2,695 & 2,722 \\
& 5 & 1,519 & 1,598 & 1,669 \\
& 8 & 1,251 & 1,260 & 1,394 \\
& 12 & 1,132 & 1,208 & 1,369 \\
\hline \multirow{4}{*}{40} & 3 & 1,408 & 1,492 & 1,541 \\
& 5 & 1,004 & 1,011 & 1,084 \\
& 8 & 0,796 & 0,852 & 1,005 \\
& 12 & 0,747 & 0,851 & 1,000 \\
\hline \multirow{4}{*}{60} & 3 & 1,112 & 1,125 & 1,130 \\
& 5 & 0,801 & 0,876 & 0,884 \\
& 8 & 0,637 & 0,714 & 0,831 \\
& 12 & 0,588 & 0,704 & 0,824 \\
\hline \multirow{4}{*}{100} & 3 & 0,815 & 0,831 & 0,879 \\
& 5 & 0,613 & 0,614 & 0,705 \\
& 8 & 0,490 & 0,548 & 0,703 \\
& 12 & 0,464 & 0,538 & 0,646 \\
\hline \multirow{4}{*}{150} & 3 & 0,657 & 0,673 & 0,735 \\
& 5 & 0,501 & 0,530 & 0,596 \\
& 8 & 0,402 & 0,444 & 0,590 \\
& 12 & 0,398 & 0,409 & 0,530 \\
\hline
\end{tabular}

Os resultados de todas as combinações em relação a $N, n_{i}$ e graus de liberdade estão apresentados na Tabela 4.4. Nota-se que o aumento na variabilidade do intercepto aleatório de $\mu$ ou equivalentemente uma diminuição dos graus de liberdade $\nu_{1}$ produz um aumento na REQM, por exemplo, para $\nu_{2}=4, N=60, n_{i}=5$ a REQM é 0,678 e 0,696 para valores de $\nu_{1}$ de 4 e 3 , respectivamente. De forma similar, o aumento na variabilidade do intercepto aleatório de $\sigma$ produz um aumento na REQM. Os menores valores da REQM são encontrados quando o número de indivíduos e o número de observações por indivíduo é grande, $N=150$ e $n_{i}=12$ dado que quando estes aumentam a REQM disminui.

\subsubsection{Cenário 3: Modelo beta exponencial potência}

Neste cenário, supomos que o modelo de regressão beta com intercepto aleatório exponencial potência segue a estrutura (4.5) com

$$
\begin{aligned}
& g\left(\mu_{i j}\right)=\beta_{11}+\beta_{21} x_{i j}+\beta_{31} t_{i}+\gamma_{i 1}, \\
& g\left(\sigma_{i j}\right)=\beta_{12}+\beta_{22} x_{i j}+\beta_{32} t_{i}+\gamma_{i 2}
\end{aligned}
$$

em que $i=1,2, \ldots, N$ e $j=1,2, \ldots, n_{i}$. Aqui consideramos valores de 0,$44 ; 0,89$ e 1,33 para os parâmetros de escala, $\lambda_{1}$ e $\lambda_{2}$, e de 1,5 para os dois parâmetros de curtose, $\nu_{1}$ e $\nu_{2}$. A variância dos interceptos aleatórios é dada por 0,$25 ; 1,0$ e 2,25, para os valores de $\lambda_{1}, \lambda_{2}, \nu_{1}$ 
Tabela 4.4: REQM das estimativas dos parâmetros do modelo beta $t$-Student com $\nu_{1}=4$ e $\nu_{1}=3$.

\begin{tabular}{|c|c|c|c|c|c|}
\hline \multirow[b]{2}{*}{$N$} & \multirow[b]{2}{*}{$n_{i}$} & \multicolumn{2}{|c|}{$\nu_{1}=4$} & \multicolumn{2}{|c|}{$\nu_{1}=3$} \\
\hline & & $\nu_{2}=4$ & $\nu_{2}=3$ & $\nu_{2}=4$ & $\nu_{2}=3$ \\
\hline \multirow{4}{*}{20} & 3 & 2,823 & 2,867 & 2,853 & 2,886 \\
\hline & 5 & 1,400 & 1,486 & 1,476 & 1,489 \\
\hline & 8 & 1,174 & 1,181 & 1,179 & 1,183 \\
\hline & 12 & 1,013 & 1,128 & 1,098 & 1,132 \\
\hline \multirow{4}{*}{40} & 3 & 1,387 & 1,389 & 1,442 & 1,451 \\
\hline & 5 & 0,886 & 0,914 & 0,890 & 0,918 \\
\hline & 8 & 0,766 & 0,784 & 0,767 & 0,793 \\
\hline & 12 & 0,750 & 0,762 & 0,763 & 0,779 \\
\hline \multirow{4}{*}{60} & 3 & 1,013 & 1,015 & 1,031 & 1,036 \\
\hline & 5 & 0,678 & 0,693 & 0,696 & 0,699 \\
\hline & 8 & 0,602 & 0,629 & 0,604 & 0,634 \\
\hline & 12 & 0,582 & 0,604 & 0,591 & 0,631 \\
\hline \multirow{4}{*}{100} & 3 & 0,727 & 0,729 & 0,740 & 0,754 \\
\hline & 5 & 0,501 & 0,520 & 0,519 & 0,543 \\
\hline & 8 & 0,451 & 0,481 & 0,468 & 0,474 \\
\hline & 12 & 0,442 & 0,451 & 0,459 & 0,460 \\
\hline \multirow{4}{*}{150} & 3 & 0,570 & 0,572 & 0,580 & 0,608 \\
\hline & 5 & 0,416 & 0,570 & 0,420 & 0,436 \\
\hline & 8 & 0,389 & 0,395 & 0,398 & 0,427 \\
\hline & 12 & 0,375 & 0,386 & 0,384 & 0,409 \\
\hline
\end{tabular}

e $\nu_{2}$ considerados.

Nota-se das Tabelas 4.5, 4.6 e 4.7 que a REQM diminui conforme o número de indivíduos e o número de observações por indivíduo aumenta para $\lambda_{1}$ e $\lambda_{2}$ fixos. Além disso, a REQM aumenta conforme o parâmetro de escala de $\sigma$ aumenta, para valores fixos de $\lambda_{1}, N$ e $n_{i}$. Observa-se também que o aumento no parâmetro de escala de $\mu$ aumenta a REQM, por exemplo, com $N=20, n_{i}=8$ e $\lambda_{2}=0,89$ a REQM é 0,907; 1,008 e 1,028 para $\lambda_{1}=0,44 ; 0,89$ e 1,33 , respectivamente. 
Tabela 4.5: REQM das estimativas dos parâmetros do modelo beta exponencial potência com $\nu_{1}=$ $\nu_{2}=1,5$ e $\lambda_{1}=0,44$.

\begin{tabular}{|c|c|c|c|c|}
\hline$N$ & $n_{i}$ & $\lambda_{2}=0,44$ & $\lambda_{2}=0,89$ & $\lambda_{2}=1,33$ \\
\hline \multirow{4}{*}{20} & 3 & 2,787 & 2,835 & 2,856 \\
\hline & 5 & 1,359 & 1,395 & 1,430 \\
\hline & 8 & 0,960 & 1,001 & 1,040 \\
\hline & 12 & 0,754 & 0,907 & 1,030 \\
\hline \multirow{4}{*}{40} & 3 & 1,322 & 1,319 & 1,326 \\
\hline & 5 & 0,808 & 0,823 & 0,886 \\
\hline & 8 & 0,583 & 0,619 & 0,682 \\
\hline & 12 & 0,508 & 0,606 & 0,618 \\
\hline \multirow{4}{*}{60} & 3 & 0,950 & 0,991 & 0,989 \\
\hline & 5 & 0,621 & 0,629 & 0,636 \\
\hline & 8 & 0,471 & 0,487 & 0,550 \\
\hline & 12 & 0,410 & 0,483 & 0,545 \\
\hline \multirow{4}{*}{100} & 3 & 0,604 & 0,670 & 0,689 \\
\hline & 5 & 0,440 & 0,454 & 0,482 \\
\hline & 8 & 0,333 & 0,383 & 0,433 \\
\hline & 12 & 0,331 & 0,380 & 0,423 \\
\hline \multirow{4}{*}{150} & 3 & 0,490 & 0,513 & 0,517 \\
\hline & 5 & 0,343 & 0,363 & 0,402 \\
\hline & 8 & 0,272 & 0,337 & 0,362 \\
\hline & 12 & 0,265 & 0,327 & 0,361 \\
\hline
\end{tabular}

\subsection{Conclusões}

O objetivo deste capítulo foi apresentar extensões dos modelos de regressão beta usuais relaxando a suposição de normalidade dos efeitos aleatórios. Nestes novos modelos a distribuição dos interceptos aleatórios assume-se que segue uma distribuição assimétrica negativa como a log-gama ou uma distribuição simétrica de caudas mais pesadas do que a normal como a $t$-Student e a exponencial potência, assim como os casos particulares Cauchy e Laplace.

Propomos um método de estimação dos parâmetros dos modelos e de predição dos interceptos aleatórios baseado numa reformulação da função de verossimilhança. Essa abordagem em conjunto com o método de Gauss-Hermite facilita a obtenção tanto das estimativas de máxima verossimilhança quanto dos melhores preditores dos interceptos aleatórios. A vantagem do método proposto neste trabalho com os trabalhos que existem nesta classe de modelos é a flexibilidade e a generalidade dado que permite acomodar diversos tipos de distribuições nos interceptos aleatórios tais como distribuições com multimodalidade e assimetria.

Os resultados do estudo de simulação mostram que, sob as condições definidas, o desempenho do processo de estimação apresenta resultados similares nos modelos beta log-gama, beta $t$-Student e beta exponencial potência. 
Tabela 4.6: $R E Q M$ das estimativas dos parâmetros do modelo beta exponencial potência com $\nu_{1}=$ $\nu_{2}=1,5$ e $\lambda_{1}=0,89$.

\begin{tabular}{ccccc}
\hline$N$ & $n_{i}$ & $\lambda_{2}=0,44$ & $\lambda_{2}=0,89$ & $\lambda_{2}=1,33$ \\
\hline \multirow{4}{*}{20} & 3 & 2,561 & 2,689 & 2,764 \\
& 5 & 1,335 & 1,399 & 1,400 \\
& 8 & 1,015 & 1,062 & 1,109 \\
& 12 & 1,005 & 1,008 & 1,107 \\
\hline \multirow{4}{*}{40} & 3 & 1,245 & 1,318 & 1,329 \\
& 5 & 0,853 & 0,861 & 0,874 \\
& 8 & 0,637 & 0,683 & 0,750 \\
& 12 & 0,563 & 0,680 & 0,740 \\
\hline \multirow{4}{*}{60} & 3 & 0,986 & 0,995 & 0,997 \\
& 5 & 0,649 & 0,651 & 0,665 \\
& 8 & 0,497 & 0,519 & 0,595 \\
& 12 & 0,452 & 0,514 & 0,536 \\
\hline \multirow{4}{*}{100} & 3 & 0,684 & 0,689 & 0,694 \\
& 5 & 0,468 & 0,481 & 0,505 \\
& 8 & 0,377 & 0,423 & 0,466 \\
& 12 & 0,351 & 0,414 & 0,464 \\
\hline \multirow{4}{*}{150} & 3 & 0,522 & 0,524 & 0,542 \\
& 5 & 0,357 & 0,389 & 0,415 \\
& 8 & 0,307 & 0,359 & 0,387 \\
& 12 & 0,288 & 0,352 & 0,367 \\
\hline
\end{tabular}

Tabela 4.7: REQM das estimativas dos parâmetros do modelo beta exponencial potência com $\nu_{1}=$ $\nu_{2}=1,5$ e $\lambda_{1}=1,33$.

\begin{tabular}{ccccc}
\hline$N$ & $n_{i}$ & $\lambda_{2}=0,44$ & $\lambda_{2}=0,89$ & $\lambda_{2}=1,33$ \\
\hline \multirow{4}{*}{20} & 3 & 2,103 & 2,121 & 2,615 \\
& 5 & 1,410 & 1,432 & 1,444 \\
& 8 & 1,061 & 1,120 & 1,170 \\
& 12 & 0,935 & 1,028 & 1,117 \\
\hline \multirow{4}{*}{40} & 3 & 1,256 & 1,290 & 1,296 \\
& 5 & 0,865 & 0,874 & 0,886 \\
& 8 & 0,691 & 0,723 & 0,803 \\
& 12 & 0,639 & 0,722 & 0,792 \\
\hline \multirow{4}{*}{60} & 3 & 1,009 & 1,754 & 1,973 \\
& 5 & 0,660 & 0,661 & 0,686 \\
& 8 & 0,553 & 0,587 & 0,625 \\
& 12 & 0,523 & 0,563 & 0,615 \\
\hline \multirow{4}{*}{100} & 3 & 0,700 & 0,700 & 0,710 \\
& 5 & 0,491 & 0,500 & 0,541 \\
& 8 & 0,426 & 0,470 & 0,522 \\
& 12 & 0,402 & 0,467 & 0,508 \\
\hline \multirow{4}{*}{150} & 3 & 0,551 & 0,553 & 0,560 \\
& 5 & 0,391 & 0,409 & 0,446 \\
& 8 & 0,342 & 0,369 & 0,417 \\
& 12 & 0,333 & 0,358 & 0,405 \\
\hline
\end{tabular}




\section{Capítulo 5}

\section{Modelos de regressão beta com intercepto e inclinação aleatória}

Neste capítulo abordamos duas extensões do modelo de regressão beta com intercepto aleatório. A primeira refere-se à inserção da inclinação aleatória ao modelo e a segunda ao ajuste do modelo com distribuições de contornos elípticos nos efeitos aleatórios.

Vários trabalhos sobre modelos de regressão beta com coeficientes de regressão aleatórios podem ser encontrados na literatura. Sob uma abordagem frequentista, Verkuilen e Smithson (2012) usam distribuições normais multivariadas para os efeitos aleatórios e por sua vez, sob uma abordagem bayesiana, Bonat et al. (2012) e Figueroa-Zúñiga et al. (2012) desenvolvem modelos com efeitos aleatórios multivariados. No contexto das distribuições elípticas, diversos autores têm usado essa classe de distribuições em modelos não lineares, ver por exemplo Russo (2010) e Meza et al. (2012), e em modelos multiníveis, Solaro e Ferrari (2007) e Manghi (2011).

Na Seção 5.1 apresentamos o modelo de regressão beta com intercepto e inclinação aleatória, assumindo efeitos aleatórios distribuídos conforme a distribuição de contornos elípticos detalhando alguns casos particulares. Na Seção 5.2 a estimação de parâmetros do modelo proposto é descrita. Um estudo de simulação supondo que os efeitos aleatórios seguem uma distribuição normal bivariada com diferentes estruturas de variância-covariância é apresentado na Seção 5.3. Finalmente, na Seção 5.4 são apresentadas as conclusões do capítulo.

\subsection{Modelo proposto}

Seja $y_{i j}$ o valor da variável resposta para o $i$-ésimo indivíduo no tempo $t_{i j}, i=1, \ldots, N$, $j=1, \ldots, n_{i}$. Assumimos que, condicional aos vetores de efeitos aleatórios $\gamma_{i 1}$ e $\gamma_{i 1}$, as respostas $y_{i 1}, y_{i 2}, \ldots y_{i n_{i}}$ são independentes e cada uma segue uma distribuição beta com função densidade de probabilidade dada em (2.8). Assumimos também que os vetores de 
efeitos aleatórios $\gamma_{i 1}$ e $\gamma_{i 1}$ são independentes entre si. O modelo de regressão beta com intercepto e inclinação aleatória assume a forma

$$
\begin{array}{r}
y_{i j} \mid \boldsymbol{\gamma}_{i 1}, \boldsymbol{\gamma}_{i 2} \stackrel{\text { ind }}{\sim} B e\left(\mu_{i j}, \sigma_{i j}\right), \\
\boldsymbol{\gamma}_{i 1} \stackrel{\text { i.i.d }}{\sim} E_{2}\left(\mathbf{0}, \boldsymbol{\Sigma}_{1}\right), \\
\boldsymbol{\gamma}_{i 2} \stackrel{\text { i.i.d }}{\sim} E_{2}\left(\mathbf{0}, \boldsymbol{\Sigma}_{2}\right),
\end{array}
$$

com $i=1,2, \ldots, N, j=1,2, \ldots, n_{i}, \gamma_{i 1}=\left(\gamma_{i 11}, \gamma_{i 21}\right)^{\top}$ e $\gamma_{i 2}=\left(\gamma_{i 12}, \gamma_{i 22}\right)^{\top}$. Os parâmetros $\mu_{i j}$ e $\sigma_{i j}$ satisfazem as seguintes relações funcionais

$$
\begin{aligned}
& g_{1}\left(\mu_{i j}\right)=\eta_{i j 1}=\boldsymbol{x}_{i j 1}^{\top} \boldsymbol{\beta}_{1}+\boldsymbol{z}_{i j 1}^{\top} \boldsymbol{\gamma}_{i 1}, \\
& g_{2}\left(\sigma_{i j}\right)=\eta_{i j 2}=\boldsymbol{x}_{i j 2}^{\top} \boldsymbol{\beta}_{2}+\boldsymbol{z}_{i j 2}^{\top} \boldsymbol{\gamma}_{i 2},
\end{aligned}
$$

em que $\boldsymbol{x}_{i j 1}=\left(x_{i j 11}, x_{i j 21}, \ldots, x_{i j p_{1} 1}\right)^{\top}$ e $\boldsymbol{x}_{i j 2}=\left(x_{i j 12}, x_{i j 22}, \ldots, x_{i j p_{2} 2}\right)^{\top}$ contêm valores das variáveis explicativas associadas aos efeitos fixos, $\boldsymbol{z}_{i j 1}$ e $\boldsymbol{z}_{i j 2}$ contêm valores das variáveis explicativas associadas aos efeitos aleatórios, $\boldsymbol{\beta}_{1}=\left(\beta_{11}, \beta_{21}, \ldots, \beta_{p_{1} 1}\right)^{\top}$ e $\boldsymbol{\beta}_{2}=\left(\beta_{12}, \beta_{22}, \ldots, \beta_{p_{2} 2}\right)^{\top}$ contêm os parâmetros desconhecidos fixos e $\gamma_{i 1}$ e $\gamma_{i 2}$ são vetores bidimensionais de efeitos aleatórios do indivíduo $i$ associados à estrutura de regressão de $\mu_{i j}$ e $\sigma_{i j}$, respectivamente. As funções de ligação $g_{1}:(0,1) \rightarrow \mathbb{R}$ e $g_{2}:(0,1) \rightarrow \mathbb{R}$ são funções monótonas crescentes e duplamente diferenciáveis. Exemplos destas funções são apresentados na Seção 3.1.

Os vetores $\gamma_{i 1}$ e $\gamma_{i 2}$ seguem uma distribuição elíptica bivariada com média 0 e matriz de escala $\Sigma_{1}$ e $\Sigma_{2}$, respectivamente. Estas matrizes são proporcionais às matrizes de variância-covariância de $\gamma_{i 1}$ e $\gamma_{i 2}$ por uma quantidade $c_{0}$ que depende da distribuição elíptica assumida. Na Seção 2.5.5 são definidos os valores $c_{0}$ para algumas distribuições que pertencem à classe de distribuições elípticas. Vale a pena ressaltar que, sob o caso normal, $c_{0}=1$.

Várias estruturas de variância-covariância podem ser especificadas para as matrizes $\boldsymbol{\Sigma}_{1}$ e $\boldsymbol{\Sigma}_{2}$, veja por exemplo Wolfinger (1993), Littell et al. (2000), Camarinha (2002) e Searle et al. (2006). No modelo (5.1) optamos por adotar as matrizes com estrutura de covariância: simples (S), de componentes de variância (CV), auto-regressiva de ordem 1 (AR-1) e de simetria composta heterogênea ( $\mathrm{SCH}$ ). Quando usamos a estrutura CV, por exemplo, os elementos das matrizes $\boldsymbol{\Sigma}_{1}$ e $\boldsymbol{\Sigma}_{2}$ são $\boldsymbol{\lambda}_{1}=\left(\lambda_{11}, \lambda_{21}\right)^{\top}$ e $\boldsymbol{\lambda}_{2}=\left(\lambda_{12}, \lambda_{22}\right)^{\top}$, respectivamente. A Tabela 5.1 apresenta as estruturas adotadas e o número de parâmetros a ser estimados. 
Tabela 5.1: Estruturas de variância-covariância

\begin{tabular}{|c|c|c|}
\hline Estrutura & Exemplo & Número de parâmetros \\
\hline S & $\left(\begin{array}{cc}\lambda & 0 \\
0 & \lambda\end{array}\right)$ & 1 \\
\hline $\mathrm{CV}$ & $\left(\begin{array}{cc}\lambda_{1} & 0 \\
0 & \lambda_{2}\end{array}\right)$ & 2 \\
\hline $\operatorname{AR}(1)$ & $\left(\begin{array}{cc}\lambda & \rho \lambda \\
\rho \lambda & \lambda\end{array}\right)$ & 2 \\
\hline $\mathrm{SCH}$ & $\left(\begin{array}{cc}\lambda_{1} & \rho \sqrt{\lambda_{1}} \sqrt{\lambda_{2}} \\
\rho \sqrt{\lambda_{1}} \sqrt{\lambda_{2}} & \lambda_{2}\end{array}\right)$ & 3 \\
\hline
\end{tabular}

A seguir serão apresentados três modelos de regressão beta com efeitos aleatórios que seguem distribuições particulares da classe de distribuições elípticas a saber: normal, $t$-Student e exponecial potência dadas em (2.14), (2.15) e (2.16), respectivamente. Estes modelos seguem a estrutura de regressão (5.1) e (5.2).

\subsubsection{Modelo beta normal}

Supondo que os $y_{i j}$ seguem uma distribuição beta com função densidade de probabilidade dada por (2.8) e os efeitos aleatórios $\gamma_{i 1}$ e $\gamma_{i 2}$ seguem uma distribuição normal com função densidade de probabilidade dada por (2.14) tem-se que o modelo beta normal com intercepto e inclinação aleatória é representado por

$$
\begin{array}{r}
y_{i j} \mid \boldsymbol{\gamma}_{i 1}, \boldsymbol{\gamma}_{i 2} \stackrel{\text { ind }}{\sim} \operatorname{Be}\left(\mu_{i j}, \sigma_{i j}\right), \\
\boldsymbol{\gamma}_{i 1} \stackrel{\text { i.i.i.d }}{\sim} N_{2}\left(\mathbf{0}, \boldsymbol{\Sigma}_{1}\right), \\
\boldsymbol{\gamma}_{i 2} \stackrel{\text { i.i.d. }}{\sim} N_{2}\left(\mathbf{0}, \boldsymbol{\Sigma}_{2}\right),
\end{array}
$$

com $i=1,2, \ldots, N$ e $j=1,2, \ldots, n_{i}$. Os parâmetros $\mu_{i j}$ e $\sigma_{i j}$ satisfazem as relações funcionais dadas em (5.2). Se $\boldsymbol{\Sigma}_{1}$ ou $\boldsymbol{\Sigma}_{2}$ é uma matriz diagonal o intercepto e a inclinação aleatória de $\mu$ ou $\sigma$ são independentes e não correlacionados.

\subsubsection{Modelo beta $t$-Student}

Considerando-se a distribuição beta com função densidade de probabilidade dada por (2.8) para $y_{i j}$ e a distribuição $t$-Student para os efeitos aleatórios $\gamma_{i 1}$ e $\gamma_{i 2}$ dada por (2.15), 
o modelo beta $t$-Student com intercepto e inclinação aleatória é dado por

$$
\begin{aligned}
& y_{i j} \mid \gamma_{i 1}, \gamma_{i 2} \stackrel{\text { ind }}{\sim} B e\left(\mu_{i j}, \sigma_{i j}\right), \\
& \gamma_{i 1} \stackrel{\text { i.i.d }}{\sim} t_{2}\left(\mathbf{0}, \boldsymbol{\Sigma}_{1}, \nu_{1}\right), \\
& \gamma_{i 2} \stackrel{\text { i.i.d }}{\sim} t_{2}\left(\mathbf{0}, \boldsymbol{\Sigma}_{2}, \nu_{2}\right),
\end{aligned}
$$

com $i=1,2, \ldots, N$ e $j=1,2, \ldots, n_{i}$. Os parâmetros $\mu_{i j}$ e $\sigma_{i j}$ satisfazem as relações funcionais dadas em (5.2). Assumindo fixos e conhecidos $\nu_{1}$ e $\nu_{2}$ o vetor de parâmetros deste modelo é dado por $\boldsymbol{\theta}=\left(\boldsymbol{\beta}_{1}^{\top}, \boldsymbol{\beta}_{2}^{\top}, \boldsymbol{\Sigma}_{1}, \boldsymbol{\Sigma}_{2}\right)^{\top}$. Caso os graus de liberdade de ambas as distribuições $t$-Student sejam um $\left(\nu_{1}=1, \nu_{2}=1\right)$ este modelo particular é denominado modelo beta Cauchy.

\subsubsection{Modelo beta exponencial potência}

Assumindo que a $y_{i j}$ segue uma distribuição beta com função densidade de probabilidade dada por (2.8) e os interceptos aleatórios $\gamma_{i 1}$ e $\gamma_{i 2}$ seguem uma distribuição exponencial potência dada por (2.16) tem-se que a representação do modelo beta exponencial potência com intercepto e inclinação aleatória é dada por

$$
\begin{aligned}
& y_{i j} \mid \boldsymbol{\gamma}_{i 1}, \boldsymbol{\gamma}_{i 2} \stackrel{\text { ind }}{\sim} \operatorname{Be}\left(\mu_{i j}, \sigma_{i j}\right), \\
& \boldsymbol{\gamma}_{i 1} \stackrel{\text { i.i.j. }}{\sim} \operatorname{EP}\left(\mathbf{0}, \boldsymbol{\Sigma}_{1}, \nu_{1}\right), \\
& \boldsymbol{\gamma}_{i 2} \stackrel{\text { i.i.i. }}{\sim} \operatorname{EP} P_{2}\left(\mathbf{0}, \boldsymbol{\Sigma}_{2}, \nu_{2}\right),
\end{aligned}
$$

com $i=1,2, \ldots, N$ e $j=1,2, \ldots, n_{i}$. Os parâmetros $\mu_{i j}$ e $\sigma_{i j}$ satisfazem as relações funcionais dadas em (5.2). Assumindo fixos e conhecidos $\nu_{1}$ e $\nu_{2}$ temos que $\boldsymbol{\theta}=\left(\boldsymbol{\beta}_{1}^{\top}, \boldsymbol{\beta}_{2}^{\top}, \boldsymbol{\Sigma}_{1}, \boldsymbol{\Sigma}_{2}\right)^{\top}$ é o vetor de parâmetros do modelo beta exponencial potência. $\mathrm{O}$ intercepto e a inclinação aleatória de $\mu$ ou $\sigma$ são independentes se e somente se $\nu_{1}=0$ ou $\nu_{2}=0$.

\subsection{Estimação}

A inferência estatística para o modelo de regressão beta com intercepto e inclinação aleatória é baseada no método de máxima verossimilhança. A seguir apresentamos as expressões da função de verossimilhança e do logaritmo da função de verossimilhança.

Seja $\boldsymbol{y}_{i}=\left(y_{i 1}, y_{i 2}, \ldots, y_{i n_{i}}\right)^{\top}$ o vetor de observações do indivíduo $i$ e $\boldsymbol{\theta}=\left(\boldsymbol{\beta}_{1}^{\top}, \boldsymbol{\beta}_{2}^{\top}, \boldsymbol{\Sigma}_{1}, \boldsymbol{\Sigma}_{2}\right)^{\top}$ o vetor de parâmetros do modelo dado em (5.1) e (5.2). Sob a representação deste modelo a função de verossimilhança para $\boldsymbol{\theta}$ baseada em $\boldsymbol{y}_{i}$ é dada por

$$
L_{i}(\boldsymbol{\theta})=\int_{\mathbb{R}^{4}} \prod_{j=1}^{n_{i}} f\left(y_{i j} \mid \boldsymbol{\gamma}_{i 1}, \boldsymbol{\gamma}_{i 2} ; \boldsymbol{\beta}_{1}, \boldsymbol{\beta}_{2}\right) \cdot f\left(\boldsymbol{\gamma}_{i 1} ; \boldsymbol{\Sigma}_{1}\right) f\left(\boldsymbol{\gamma}_{i 2} ; \boldsymbol{\Sigma}_{2}\right) d \boldsymbol{\gamma}_{i 1} d \boldsymbol{\gamma}_{i 2}
$$


em que $f\left(\boldsymbol{\gamma}_{i 1} ; \boldsymbol{\Sigma}_{1}\right)$ e $f\left(\boldsymbol{\gamma}_{i 2} ; \boldsymbol{\Sigma}_{2}\right)$ são as funções densidade de probabilidades de $\boldsymbol{\gamma}_{i 1}$ e $\boldsymbol{\gamma}_{i 2}$, respectivamente.

Como descrevemos na Seção 4.2, para maximizar o logaritmo da função de verossimilhança $\ell(\boldsymbol{\theta})$ reformulamos $L_{i}(\theta)$ multiplicando e dividindo o integrando por duas densidades normais padrão bivariadas. Liu e Yu (2008) usaram esta abordagem em modelos lineares generalizados mistos com efeitos aleatórios bivariados. Seguindo esta metodologia temos que

$$
L_{i}(\boldsymbol{\theta})=\int_{\mathbb{R}^{4}} \prod_{j=1}^{n_{i}} f\left(y_{i j} \mid \boldsymbol{\gamma}_{i 1}, \boldsymbol{\gamma}_{i 2} ; \boldsymbol{\beta}_{1}, \boldsymbol{\beta}_{2}\right) \cdot \frac{f\left(\boldsymbol{\gamma}_{i 1} ; \boldsymbol{\Sigma}_{1}\right)}{\phi\left(\boldsymbol{\gamma}_{i 1}\right)} \frac{f\left(\boldsymbol{\gamma}_{i 2} ; \boldsymbol{\Sigma}_{2}\right)}{\phi\left(\boldsymbol{\gamma}_{i 2}\right)} \phi\left(\boldsymbol{\gamma}_{i 1}\right) \phi\left(\boldsymbol{\gamma}_{i 2}\right) d \boldsymbol{\gamma}_{i 1} d \boldsymbol{\gamma}_{i 2}
$$

Uma vez reformulada $L_{i}(\boldsymbol{\theta})$ obtemos a função de verossimilhança para $\boldsymbol{\theta}$ baseada em $\boldsymbol{y}$ como segue

$$
L(\boldsymbol{\theta})=\prod_{i=1}^{N} \int_{\mathbb{R}^{4}} \prod_{j=1}^{n_{i}} f\left(y_{i j} \mid \boldsymbol{\gamma}_{i 1}, \boldsymbol{\gamma}_{i 2} ; \boldsymbol{\beta}_{1}, \boldsymbol{\beta}_{2}\right) \cdot \frac{f\left(\boldsymbol{\gamma}_{i 1} ; \boldsymbol{\Sigma}_{1}\right)}{\phi\left(\boldsymbol{\gamma}_{i 1}\right)} \frac{f\left(\boldsymbol{\gamma}_{i 2} ; \boldsymbol{\Sigma}_{2}\right)}{\phi\left(\boldsymbol{\gamma}_{i 2}\right)} \phi\left(\boldsymbol{\gamma}_{i 1}\right) \phi\left(\boldsymbol{\gamma}_{i 2}\right) d \boldsymbol{\gamma}_{i 1} d \boldsymbol{\gamma}_{i 2}
$$

e o logaritmo da função de verossimilhança toma a forma

$$
\ell(\boldsymbol{\theta})=\sum_{i=1}^{N} \log \left(\int_{\mathbb{R}^{4}} \prod_{j=1}^{n_{i}} f\left(y_{i j} \mid \boldsymbol{\gamma}_{i 1}, \boldsymbol{\gamma}_{i 2} ; \boldsymbol{\beta}_{1}, \boldsymbol{\beta}_{2}\right) \cdot \frac{f\left(\boldsymbol{\gamma}_{i 1} ; \boldsymbol{\Sigma}_{1}\right)}{\phi\left(\boldsymbol{\gamma}_{i 1}\right)} \frac{f\left(\boldsymbol{\gamma}_{i 2} ; \boldsymbol{\Sigma}_{2}\right)}{\phi\left(\boldsymbol{\gamma}_{i 2}\right)} \phi\left(\boldsymbol{\gamma}_{i 1}\right) \phi\left(\boldsymbol{\gamma}_{i 2}\right) d \boldsymbol{\gamma}_{i 1} d \boldsymbol{\gamma}_{i 2}\right)
$$

A aproximação da função de verossimilhança pode ser feita por meio da quadratura de Gauss-Hermite multidimensional. As funções GHQ e blmmML do pacote BLMM implementado no $R$ podem ser usadas para calcular os pontos e pesos da quadratura e obter as estimativas de máxima verossimilhança do modelo (5.1), respectivamente.

\subsection{Estudo de simulação}

Nesta seção apresentamos um estudo de simulação a fim de avaliar o desempenho do processo de estimação. Consideramos diferentes cenários com o objetivo de analisar o impacto do número de indivíduos e o número de observações por indivíduo. Alem disso, consideramos quatro estruturas de variância-covariância, descritas na Tabela 5.1, para a distribuição bivariada dos efeitos aleatórios.

Para cada cenário, 10000 réplicas foram geradas a partir de um modelo de regressão beta com efeitos aleatórios normais em que a estrutura de regressão para $\mu$ é dada pelo intercepto e a inclinação aleatória e a estrutura de regressão para $\sigma$ difere no componente aleatório, como é descrito nas Seções 5.3.1 a 5.3.3. A função de ligação usada para $\mu$ e $\sigma$ foi a logito. O número de indivíduos considerados foi $N=15,20$ e 30 e o número de observações por indivíduo foi $n_{i}=4,6,8$ e 10. Seguindo a estrutura de geração de dados de Bonat et al. 
(2012) a variável tempo foi obtida de acordo com a expressão $t_{i j 1}=t_{i j 2}=(j-1) /\left(n_{i}-1\right)$.

Para avaliar o desempenho do processo de estimação usamos a raiz do erro quadrático médio (REQM) definida em (3.22). Cada um dos três cenários são descritos a seguir.

\subsubsection{Cenário 1}

Neste cenário consideramos um modelo de regressão beta com efeitos aleatórios normais na estrutura de regressão de $\mu$ como segue

$$
\begin{aligned}
y_{i j} \mid \gamma_{i 1} & \stackrel{\text { ind }}{\sim} B e\left(\mu_{i j}, \sigma_{i j}\right), \\
\boldsymbol{\gamma}_{i 1} & \stackrel{\text { i.i.d. }}{\sim} N_{2}\left(\mathbf{0}, \boldsymbol{\Sigma}_{1}\right),
\end{aligned}
$$

com

$$
g\left(\mu_{i j}\right)=\left(\beta_{11}+\gamma_{i 11}\right)+\left(\beta_{21}+\gamma_{i 21}\right) t_{i j 1}
$$

em que $i=1, \ldots, N$ e $j=1, \ldots, n_{i}$. mOs parâmetros deste modelo são $\boldsymbol{\beta}_{1}=\left(\beta_{11}, \beta_{21}\right)^{\top}$, $\beta_{12}, \boldsymbol{\lambda}_{1}=\left(\lambda_{11}, \lambda_{21}\right)^{\top}$ e $\rho_{1}$. Os valores considerados nas simulações foram $\boldsymbol{\beta}_{1}=(-0,3 ; 0,6)^{\top}$, $\beta_{12}=-2,0 ; \boldsymbol{\lambda}_{1}=(0,5 ; 0,3)^{\top}$ e $\rho_{1}=-0,5$.

\subsubsection{Cenário 2}

No cenário 2 assumimos um modelo de regressão beta com intercepto e inclinação aleatória na estrutura de regressão de $\mu$ e com intercepto aleatório na estrutura de regressão de $\sigma$ como segue

$$
\begin{aligned}
y_{i j} \mid \gamma_{i 1}, \gamma_{i 2} & \stackrel{\text { ind }}{\sim} B e\left(\mu_{i j}, \sigma_{i j}\right), \\
\gamma_{i 1} & \stackrel{\text { i.i.d }}{\sim} N_{2}\left(\mathbf{0}, \boldsymbol{\Sigma}_{1}\right), \\
\gamma_{i 2} & \stackrel{\text { i.i.d. }}{\sim} N\left(0, \lambda_{12}\right),
\end{aligned}
$$

com

$$
\begin{aligned}
& g\left(\mu_{i j}\right)=\left(\beta_{11}+\gamma_{i 11}\right)+\left(\beta_{21}+\gamma_{i 21}\right) t_{i j 1}, \\
& g\left(\sigma_{i j}\right)=\left(\beta_{12}+\gamma_{i 12}\right)
\end{aligned}
$$

em que $i=1, \ldots, N$ e $j=1, \ldots, n_{i}$. Os parâmetros do modelo considerado neste cenário são $\boldsymbol{\beta}_{1}=\left(\beta_{11}, \beta_{21}\right)^{\top}, \beta_{12}, \boldsymbol{\lambda}_{1}=\left(\lambda_{11}, \lambda_{21}\right)^{\top}, \lambda_{12}$ e $\rho_{1}$. Usamos os seguintes valores para os parâmetros: $\boldsymbol{\beta}_{1}=(-0,3 ; 0,6)^{\top}, \beta_{12}=-2,0 ; \boldsymbol{\lambda}_{1}=(0,5 ; 0,3)^{\top}, \lambda_{12}=0,3$ e $\rho_{1}=-0,5$. 


\subsubsection{Cenário 3}

No cenário 3 supomos um modelo de regressão beta normal com intercepto e inclinação aleatória em $\mu$ e $\sigma$ como segue

$$
\begin{aligned}
& y_{i j} \mid \gamma_{i 1}, \gamma_{i 2} \stackrel{\text { ind }}{\sim} B e\left(\mu_{i j}, \sigma_{i j}\right), \\
& \gamma_{i 1} \stackrel{\text { i.i.i. }}{\sim} N_{2}\left(\mathbf{0}, \boldsymbol{\Sigma}_{1}\right), \\
& \gamma_{i 2} \stackrel{\text { i.i.d }}{\sim} N_{2}\left(\mathbf{0}, \boldsymbol{\Sigma}_{2}\right),
\end{aligned}
$$

com

$$
\begin{aligned}
& g\left(\mu_{i j}\right)=\left(\beta_{11}+\gamma_{i 11}\right)+\left(\beta_{21}+\gamma_{i 21}\right) t_{i j 1}, \\
& g\left(\sigma_{i j}\right)=\left(\beta_{12}+\gamma_{i 12}\right)+\left(\beta_{22}+\gamma_{i 22}\right) t_{i j 2},
\end{aligned}
$$

em que $i=1, \ldots, N$ e $j=1, \ldots, n_{i}$. Os parâmetros do modelo suposto neste cenário são $\boldsymbol{\beta}_{1}=\left(\beta_{11}, \beta_{21}\right)^{\top}, \boldsymbol{\beta}_{2}=\left(\beta_{12}, \beta_{22}\right)^{\top}, \boldsymbol{\lambda}_{1}=\left(\lambda_{11}, \lambda_{21}\right)^{\top}, \boldsymbol{\lambda}_{2}=\left(\lambda_{12}, \lambda_{22}\right)^{\top}, \rho_{1}$ e $\rho_{2}$. Os valores considerados nas simulações foram $\boldsymbol{\beta}_{1}=(-0,3 ; 0,6)^{\top}, \boldsymbol{\beta}_{2}=(-2,0 ;-1,5)^{\top}, \boldsymbol{\lambda}_{1}=(0,5 ; 0,3)^{\top}$, $\boldsymbol{\lambda}_{2}=(0,3 ; 0,5)^{\top}, \rho_{1}=-0,5$ e $\rho_{2}=-0,5$.

As Tabelas 5.2, 5.3 e 5.4 mostram os resultados das simulações dos três cenários. Nota-se que a REQM diminui com o aumento do número de indivíduos e do número de observações por indivíduo, para uma determinada estrutura de variância-covariância. Por exemplo, no cenário 1 quando o número de observações por indivíduo é $n_{i}=8$ e a estrutura de variância-covariância é $\mathrm{AR}(1)$, a REQM é 0,628; 0,550 e 0,453, para número de indivíduos de $N=15,20$ e 30 , respectivamente.

Da Tabela 5.2 nota-se que o aumento do número de parâmetros na estrutura de variânciacovariância aumenta o valor da REQM. A Tabela 5.4 mostra que a inclusão de componentes aleatórios, intercepto e inclinação, na estrutura de regressão de $\sigma$ aumenta o valor da REQM.

\subsection{Conclusões}

Neste capítulo abordamos duas extensões do modelo de regressão beta usual, a inclusão da inclinação aleatória na estrutura de regressão dos parâmetros e o uso de distribuições de contornos elípticos na distribuição dos efeitos aleatórios. Nestes modelos consideramos também estruturas de variância-covariância para as matrizes de escala das distribuições consideradas. O estudo de simulação realizado mostrou que para o número de indivíduos considerados o processo de estimação dos parâmetros obtém os melhores resultados quando o número de parâmetros a ser estimados é pequeno. 
Tabela 5.2: REQM das estimativas dos parâmetros do modelo beta normal considerado no cenário 1.

\begin{tabular}{cccccc}
\hline & & \multicolumn{3}{c}{ Estruturas de variância-covariância } \\
\cline { 3 - 6 }$N$ & $n_{i}$ & $\mathrm{~S}$ & $\mathrm{CV}$ & $\mathrm{AR} 1$ & $\mathrm{SCH}$ \\
\hline \multirow{4}{*}{15} & 4 & 0,710 & 0,724 & 0,765 & 0,782 \\
& 6 & 0,619 & 0,671 & 0,722 & 0,835 \\
& 8 & 0,512 & 0,624 & 0,628 & 0,723 \\
& 10 & 0,493 & 0,542 & 0,600 & 0,660 \\
\hline \multirow{4}{*}{20} & 4 & 0,652 & 0,738 & 0,747 & 0,776 \\
& 6 & 0,587 & 0,599 & 0,740 & 0,770 \\
& 8 & 0,462 & 0,549 & 0,550 & 0,608 \\
& 10 & 0,444 & 0,484 & 0,530 & 0,555 \\
\hline & 4 & 0,626 & 0,668 & 0,702 & 0,763 \\
30 & 6 & 0,520 & 0,592 & 0,651 & 0,665 \\
& 8 & 0,401 & 0,453 & 0,454 & 0,533 \\
& 10 & 0,377 & 0,397 & 0,447 & 0,485 \\
\hline
\end{tabular}

Tabela 5.3: REQM das estimativas dos parâmetros do modelo beta normal considerado no cenário 2 .

\begin{tabular}{cccccr}
\hline & & \multicolumn{3}{c}{ Estruturas de variância-covariância } \\
\cline { 3 - 6 }$N$ & $n_{i}$ & $\mathrm{~S}$ & $\mathrm{CV}$ & $\mathrm{AR} 1$ & $\mathrm{SCH}$ \\
\hline \multirow{4}{*}{15} & 4 & 1,102 & 1,097 & 1,112 & 1,118 \\
& 6 & 0,974 & 0,958 & 0,996 & 0,959 \\
& 8 & 0,807 & 0,793 & 0,855 & 0,867 \\
& 10 & 0,614 & 0,702 & 0,714 & 0,765 \\
\hline \multirow{4}{*}{20} & 4 & 0,982 & 0,909 & 1,000 & 0,959 \\
& 6 & 0,900 & 0,845 & 0,903 & 0,852 \\
& 8 & 0,780 & 0,733 & 0,749 & 0,768 \\
& 10 & 0,531 & 0,645 & 0,621 & 0,688 \\
\hline & 4 & 0,845 & 0,748 & 0,880 & 0,803 \\
30 & 6 & 0,829 & 0,663 & 0,835 & 0,706 \\
& 8 & 0,709 & 0,654 & 0,661 & 0,638 \\
& 10 & 0,445 & 0,533 & 1,000 & 0,557 \\
\hline
\end{tabular}


Tabela 5.4: REQM das estimativas dos parâmetros do modelo beta normal considerado no cenário 3.

\begin{tabular}{cccccr}
\hline & & \multicolumn{3}{c}{ Estruturas de variância-covariância } \\
\cline { 3 - 6 }$N$ & $n_{i}$ & $\mathrm{~S}$ & $\mathrm{CV}$ & $\mathrm{AR} 1$ & $\mathrm{SCH}$ \\
\hline \multirow{4}{*}{15} & 4 & 1,044 & 1,287 & 1,249 & 1,762 \\
& 6 & 1,020 & 1,169 & 1,172 & 1,636 \\
& 8 & 0,951 & 1,133 & 1,095 & 1,557 \\
& 10 & 0,845 & 1,085 & 1,155 & 1,644 \\
\hline & 4 & 1,002 & 1,114 & 1,088 & 1,491 \\
20 & 6 & 0,938 & 1,024 & 1,009 & 1,337 \\
& 8 & 0,844 & 0,955 & 0,971 & 1,294 \\
& 10 & 0,724 & 0,882 & 0,947 & 1,313 \\
\hline & 4 & 0,850 & 0,942 & 0,927 & 1,232 \\
30 & 6 & 0,822 & 0,900 & 0,877 & 1,103 \\
& 8 & 0,742 & 0,862 & 0,769 & 1,071 \\
& 10 & 0,604 & 0,735 & 0,753 & 1,025 \\
\hline
\end{tabular}


52 MODELOS DE REGRESSÃO BETA COM INTERCEPTO E INCLINAÇÃO ALEATÓRIA 5.4 


\section{Capítulo 6}

\section{Seleção de modelos e análise de resíduos}

A seleção de modelos surge quando há um grupo de modelos candidatos para o mesmo conjunto de dados e fornece apenas um passo de muitos na escolha do modelo mais adequado. Outros passos incluem a redução do modelo usando testes de hipóteses e a avaliação do ajuste do modelo usando ferramentas de diagnóstico apropriadas como, por exemplo, a análise de resíduos.

A análise de resíduos é usada para detectar a presença de observações atípicas e avaliar as suposições do modelo, pode incluir gráficos informais para apresentar características gerais dos resíduos, bem como testes formais para detectar afastamentos específicos as suposiçoes, assim os resíduos podem ser vistos como medidas de concordância entre os dados e o modelo ajustado. No modelo de regressão beta vários tipos de resíduos têm sido propostos. Ferrari e Cribari-Neto (2004) propõem o resíduo deviance e o resíduo padronizado, Espinheira et al. (2008) propõem o resíduo ponderado e o resíduo ponderado padronizado, obtidos da convergência do processo iterativo escore de Fisher para a estimação dos parâmetros de regressão. Rocha e Simas (2011) sugerem utilizar resíduos padronizados, escore, escore padronizado e escore modificado baseados num modelo de regressão que generaliza o modelo de Ferrari e Cribari-Neto (2004). Anholeto (2010) propõe resíduos de Pearson melhorados baseados nos trabalhos de Cox (1968), Cordeiro (2004) e Simas e Cordeiro (2009). Por sua vez, nos modelos lineares mistos, Hilden-Minton (1995), Verbeke e Lesaffre (1996), Pinheiro e Bates (2000) e Nobre e Singer (2007) propuseram resíduos marginais, condicionais, condicionais padronizados e aleatórios para analisar modelos que contêm mais de uma fonte de variação. Num caso mais geral Dunn e Smyth (1996) propõem o resíduo quantil aleatorizado para modelos de regressão com respostas independentes.

Neste capítulo propomos um procedimento de seleção do modelos de regressão beta com efeitos aleatórios baseado no método de seleção de variáveis de Ryoo (2010) e no método de seleção de modelos de Stasinopoulos et al. (2012). Também são propostos resíduos para estes modelos. 


\subsection{Seleção de modelos}

O critério mais usado para comparar modelos encaixados é o teste de razão de verossimilhanças que é baseado no máximo da função de verossimilhança. Existem outras ferramentas de seleção de modelos que são amplamente usadas, tais como o Critério de Informação de Akaike AIC, (Akaike, 1974), o critério de informação bayesiano BIC, (Schwarz, 1978), o critério de informação de Hannan-Quin HQIC, (Hannan e Quin, 1979), o AIC consistente CAIC, (Bozdogan, 1987) e o AIC corrigido AICC, (Hurvich e Tsai, 1989), bem como várias versões destes critérios. De acordo com as suas propriedades assintóticas os critérios de informação podem ser classificados em duas categorias, os critérios de eficiência, tais como os critérios AIC ou AICC, e os critérios consistentes, tais como os critérios CAIC, BIC ou HQIC.

Para selecionar os modelos de regressão beta com efeitos aleatórios para dados longitudinais neste trabalho propomos uma metodologia que combina os métodos de seleção nos modelos GAMLSS e nos modelos lineares mistos com dados longitudinais propostos por Stasinopoulos et al. (2012) e Ryoo (2010), respectivamente. O método de seleção dos modelos GAMLSS permite obter as estruturas de regressão dos parâmetros do modelo por meio do teste de razão de verossimilhanças generalizado ou do critério AIC generalizado (GAIC) que estão baseados na função desvio. Por sua vez, o método de seleção de modelos proposto por Ryoo (2010) permite obter os efeitos fixos e aleatórios de um modelo linear misto considerando a estrutura longitudinal dos dados por meio do teste de razão de verossimilhanças. $\mathrm{Na}$ seleção dos modelos de regressão beta com efeitos aleatórios usaremos o Critério de Informação de Akaike (AIC), definido como

$$
A I C=2 \hat{\ell}+2 p
$$

em que $\hat{\ell}$ é o logaritmo da função de verossimilhança avaliado nas estimativas de máxima verossimilhança e $p$ é o número de parâmetros do modelo. O modelo escolhido é o modelo com menor AIC.

A metodologia de seleção dos modelos de regressão beta com efeitos aleatórios é definida pela seleção das estruturas de regressão para $\mu$ e $\sigma$ e dos efeitos fixos e aleatórios em cada uma destas estruturas. A seguir descrevemos em detalhe a metodologia proposta.

\subsubsection{Seleção da estrutura de regressão de $\mu$ e $\sigma$}

Para selecionar a estrutura de regressão de $\mu$ e $\sigma$ consideramos três passos em que a estimação é realizada de maneira conjunta. Nesses passos usamos as abordagens I e II, descritas em detalhes na próxima seção. Os passos são os seguintes

1. Construir um modelo para $\mu$ usando a abordagem I. O parâmetro $\sigma$ é estimado sem considerar variáveis explicativas. 
2. Dado o modelo para $\mu$ construir um modelo para $\sigma$ usando a abordagem I.

3. Dado o modelo para $\sigma$ verificar se os termos do modelo em $\mu$ são necesários usando a abordagem II.

Se os modelos obtidos no passo 2 e 3 são diferentes será selecionado o modelo com menor AIC. No caso de que os modelos apresentem o mesmo valor do AIC será aplicado o princípio da parcimônia que seleciona o modelo com menor número de parâmetros.

Para conjuntos pequenos de dados, a amostra de dados pode ser utilizada tanto para o ajuste do modelo quanto para a seleção do modelo. Para conjuntos de dados de maior dimensão, os dados podem ser divididos em conjuntos de dados de treinamento, validação e teste. Os dados de treinamento podem ser usados para o ajuste do modelo, os de validação para a seleção do modelo e os de teste para a avaliação do poder preditivo do modelo escolhido.

\subsubsection{Seleção de efeitos fixos e aleatórios}

As seguintes duas abordagens propostas permitem selecionar os termos de efeitos fixos e efeitos aleatórios dos modelos de regressão de $\mu$ e $\sigma$. Assumiremos que o máximo grau do polinômio na variável tempo é o número de observações repetidas menos um ou o número mínimo de observações repetidas menos um, se o estudo é não balanceado.

\section{Abordagem I}

Nesta abordagem o procedimento de seleção é definido pelos seguintes passos

1. Inicie com um modelo de intercepto aleatório: o modelo de regressão beta mais simples que consideramos é o modelo de intercepto aleatório. Este passo permite avaliar a variabilidade da variável resposta por meio apenas da variabilidade existente entre indivíduos, sem considerar o efeito das variáveis explicativas. O modelo é denotado como M1.

2. Adicione a variável tempo: neste passo realizamos um procedimento de seleção para frente (forward) para selecionar o grau do polinômio da variável tempo no modelo. $\mathrm{O}$ procedimento termina quando a inclusão de um polinômio gera um AIC maior que o AIC do modelo M1. O modelo resultante é denotado como M2.

3. Adicione variáveis explicativas: para adicionar variáveis explicativas no modelo realizamos um procedimento de seleção forward. O procedimento termina quando o AIC gerado pela inclusão de uma variável explicativa é maior do que o AIC do modelo M2. O modelo final é denotado por M3. 
4. Adicione interações entre o tempo e as variáveis explicativas: Utilizamos o procedimento de seleção forward para obter as interações entre o tempo e as variáveis explicativas. O modelo é denotado como M4.

5. Adicione efeitos aleatórios: neste passo estudamos a inclusão da inclinação aleatória na estrutura de regressão. O intuito deste passo é investigar a variação entre indivíduos. Se o AIC gerado pela inclusão da inclinação aleatória é menor do que o AIC do modelo M4 incluímos este efeito aleatório no modelo e posteriormente selecionamos a estrutura de variância-covariância dos efeitos aleatórios.

\section{Abordagem II}

A seleção dos efeitos fixos e aleatórios é feita com os seguintes passos

1. Inicie com um modelo de intercepto aleatório saturado: O modelo de intercepto aleatório saturado contém o polinômio de máximo grau na variável tempo. O modelo é denotado como M1.

2. Retire termos do polinômio do tempo: realizamos um procedimento de seleção para trás (backward) para escolher o grau do polinômio na variável tempo. O procedimento acaba quando a eliminação de um polinômio gera um AIC maior do que o AIC do modelo M1. O modelo final é denotado como M2.

3. Adicione as variáveis explicativas: para selecionar as variáveis explicativas usamos um procedimento de seleção forward, de forma similar ao passo 3 da abordagem I. $\mathrm{O}$ modelo obtido é denotado como M3.

4. Adicione interações entre o tempo e as variáveis explicativas: realizamos um procedimento de seleção forward para selecionar as interações entre o tempo e as variáveis explicativas, como no passo 4 da abordagem I. O modelo final é denotado como M4.

5. Adicione efeitos aleatórios: se o AIC gerado com a inclusão da inclinação aleatória gera um AIC menor que o AIC do modelo M4 incluímos este efeito aleatório no modelo e selecionamos a estrutura de variância-covariância dos efeitos aleatórios.

É interessante notar que as duas abordagens são diferentes apenas no passos 1 e 2 . O resto do procedimento de construção do modelo é idêntico. No entanto, os passos 3, 4 e 5 são condicionados pelo resultado dos passos 1 e 2, que distinguem uma abordagem da outra. Esta proposta de seleção de modelos será utilizada no Capítulo 7. 


\subsection{Resíduos}

A seguir apresentamos os resíduos quantil aleatorizado, condicional, marginal e aleatório para o modelo de regressão beta com interceptos aleatórios. Além disso, descrevemos a construção do gráfico de probabilidade meio normal com envelope simulado.

\subsubsection{Resíduo quantil aleatorizado}

O resíduo quantil aleatorizado tem como objetivo avaliar a adequação geral do modelo aos dados e é definido como

$$
r_{q_{i j}}=\Phi^{-1}\left(F\left(y_{i j} ; \hat{\mu}_{i j}, \hat{\sigma}_{i j}\right)\right)
$$

em que $i=1,2, \ldots, N, \Phi^{-1}$ é a função inversa da função distribuição acumulada de uma variável com distribuição normal padrão e $F\left(y_{i j}\right)$ é a função distribuição acumulada de uma variável com distribuição beta $B e(\mu, \sigma)$.

\subsubsection{Resíduo condicional}

O resíduo condicional padronizado incorpora o efeito da presença de observações atípicas e é dado por

$$
r_{c_{i j}}=\frac{y_{i j}-\widehat{\mathrm{E}}\left(y_{i j} \mid \gamma_{i 1}, \gamma_{i 2}\right)}{\sqrt{\widehat{\operatorname{Var}}\left(y_{i j} \mid \gamma_{i 1}, \gamma_{i 2}\right)}},
$$

em que $\widehat{\mathrm{E}}\left(y_{i j} \mid \gamma_{i 1}, \gamma_{i 2}\right)=\hat{\mu}_{i j}$ e $\widehat{\operatorname{Var}}\left(y_{i j} \mid \gamma_{i 1}, \gamma_{i 2}\right)=\hat{\sigma}_{i j}^{2} \hat{\mu}_{i j}\left(1-\hat{\mu}_{i j}\right)$ e $\hat{\mu}_{i j}$ e $\hat{\sigma}_{i j}$ são calculados como $\hat{\mu}_{i j}=g_{1}^{-1}\left(\boldsymbol{x}_{i j 1}^{T} \hat{\boldsymbol{\beta}}_{1}+\hat{\gamma}_{i 1}\right)$ e $\hat{\sigma}_{i j}=g_{2}^{-1}\left(\boldsymbol{x}_{i j 2}^{T} \hat{\boldsymbol{\beta}}_{2}+\hat{\gamma}_{i 2}\right)$. Os valores $\hat{\boldsymbol{\beta}}_{1}$ e $\hat{\boldsymbol{\beta}}_{2}$ são os estimadores de máxima verossimilhança de $\boldsymbol{\beta}_{1}$ e $\boldsymbol{\beta}_{2}$ e $\widetilde{\gamma}_{i 1}$ e $\widetilde{\gamma}_{i 2}$ são os melhores preditores de $\gamma_{i 1}$ e $\gamma_{i 2}$.

\subsubsection{Resíduo marginal}

O resíduo marginal padronizado incorpora o efeito da presença de observações atípicas e pode ser escrito como

$$
r_{\mathrm{m} i j}=\frac{y_{i j}-\widehat{\mathrm{E}}\left(y_{i j}\right)}{\sqrt{\widehat{\operatorname{Var}}\left(y_{i j}\right)}},
$$

em que $\widehat{\mathrm{E}}\left(y_{i j}\right)=\mathrm{E}\left(\hat{\mu}_{i j}\right)$ e $\widehat{\operatorname{Var}}\left(y_{i j}\right)=\mathrm{E}\left(\hat{\mu}_{i j}^{2}\right)-\left(\mathrm{E}\left(\hat{\mu}_{i j}\right)\right)^{2}+\mathrm{E}\left(\hat{\sigma}_{i j}^{2}\right) \cdot \mathrm{E}\left(\hat{\mu}_{i j}\left(1-\hat{\mu}_{i j}\right)\right)$.

\subsubsection{Resíduo aleatório}

Os resíduos aleatórios para $\mu$ e $\sigma$ exploram a presença de indivíduos atípicos e são definidos como

$$
r_{a_{\mu}}=\frac{\widetilde{\gamma}_{i 1}}{\sqrt{\widehat{\operatorname{Var}}\left(\gamma_{i 1}\right)}}
$$




$$
r_{a_{\sigma}}=\frac{\widetilde{\gamma}_{i 2}}{\sqrt{\widehat{\operatorname{Var}}\left(\gamma_{i 2}\right)}},
$$

com $i=1,2, \ldots, N$, em que $\widetilde{\gamma}_{i 1}$ e $\widetilde{\gamma}_{i 2}$ são os melhores preditores de $\gamma_{i 1}$ e $\gamma_{i 2}$ e $\widehat{\operatorname{Var}}\left(\gamma_{i 1}\right)$ e $\widehat{\operatorname{Var}}\left(\gamma_{i 2}\right)$ são as variâncias estimadas da distribuição dos efeitos aleatórios $\gamma_{i 1}$ e $\gamma_{i 2}$, respectivamente.

\subsubsection{Envelope simulado}

Para cada um dos resíduos descritos anteriormente é possível a construção do gráfico de probabilidade meio-normal com envelope simulado que permite identificar observações atípicas e avaliar a suposição sobre a distribuição da variável resposta.

Atkinson (1985) e Kutner et al. (2005) sugerem usar o gráfico de probabilidade meionormal com envelope simulado como uma ferramenta de diagnóstico. Para a construção do gráfico dispomos do $k$-ésimo $(k=1, \ldots, n)$ valor ordenado do resíduo versus o correspondente valor esperado da estatística de ordem, em valor absoluto, da distribuição $N(0,1)$, dado por

$$
\Phi^{-1}\left(\frac{k+n-1 / 8}{2 n+1 / 2}\right)
$$

em que $\Phi(\cdot)$ é a função de distribuição acumulada da $N(0,1)$. Esse gráfico pode ser usado sem que os resíduos apresentem distribuição normal.

A construção do gráfico de probabilidade meio-normal com envelope simulado segue os seguintes passos:

1. Simule uma amostra de $n$ observações independentes para a variável resposta beta usando a informação do modelo ajustado aos dados originais.

2. Ajuste, às respostas beta simuladas no passo 1, o mesmo modelo ajustado aos dados originais.

3. Calcule o resíduo proposto e ordene os seus valores absolutos.

4. Repita os passos 1 a $3 k$ vezes.

5. Calcule o mínimo, a média e o máximo dos $k$ resíduos.

6. Disponha no gráfico de probabilidade meio normal do resíduo proposto os valores mínimos, médios e máximos dos dados simulados versus os correspondentes valores esperados em (6.6) e conecte os pontos para formar as bandas de confiança.

Segundo Atkinson (1985) é suficiente usar $k=19$. As observações correspondentes aos resíduos fora das bandas de confiança devem ser identificadas e investigadas. Além disso, se há 
uma proporção considerável de pontos fora das bandas é evidência de que o modelo ajustado não é adequado.

Para gerar os gráficos de probabilidade meio normal dos resíduos quantil aleatorizado, condicional, marginal e aleatório foi implementada a função hal fnorm do pacote BLMM, descrita no Capítulo 8. Estes gráficos são usados nas aplicações apresentadas no Capítulo 7

\subsubsection{Distribuição empírica dos resíduos}

Esta seção tem como objetivo estudar o comportamento das distribuições empíricas dos resíduos quantil aleatorizado, condicional, marginal e aleatórios, definidos em (6.1), (6.2), (6.3), (6.4) e (6.5), respectivamente. Consideramos as simulações realizadas na Seções 3.4 e 4.4 e os modelos de regressão beta com intercepto aleatório normal, log-gama, $t$-Student e exponencial potência.

Em cada réplica dos estudos de simulação foram obtidas as estimativas de máxima verossimilhança dos componentes de $\boldsymbol{\theta}$ de cada modelo e o melhor preditor dos interceptos aleatórios de $\mu$ e $\sigma$. Esses valores foram usados para calcular os resíduos $r_{q}, r_{c}, r_{a \mu}$ e $r_{a \sigma}$. Além disso, foram calculadas a média e a variância da distribuição marginal de $\boldsymbol{y}$ e estas foram usadas para obter o resíduo $r_{m}$.

As Figuras 6.1 e 6.4 apresentam gráficos de probabilidade normal dos resíduos quantil aleatorizado, condicional, marginal e aleatórios para o modelo beta normal dado na equação (3.20) quando $N=20, n_{i}=3,5,8$ e $12, \lambda_{1}=0,5$ e $\lambda_{2}=0,5 ; 1,0$ e 1,5. Cada painel nos gráficos apresenta a distribuição empírica dos resíduos para um caso particular, por exemplo em cada um dos três painéis inferiores da Figura 6.1 são apresentadas as distribuições dos resíduos quantil aleatorizado, condicional e marginal quando $N=20, n_{i}=5, \lambda_{1}=0,5$ e $\lambda_{2}=0,5 ; 1,0$ e 1,5 , respectivamente.

As figuras mostram que o resíduo quantil aleatorizado segue uma distribuição aproximadamente normal. Os resíduos condicional e marginal não seguem uma distribuição normal, provavelmente seguem uma distribuição de caudas mais pesadas como a t-Student. Além disso, nota-se dos gráficos de probabilidade normal dos resíduos aleatórios para $\mu$ e $\sigma$ que a distribuição de ambos resíduos é aproximadamente normal.

As Figuras 6.5 a 6.8 mostram gráficos de probabilidade normal dos resíduos quantil aleatorizado, condicional, marginal e aleatórios para o modelo beta log-gama expresso na equação (4.3) quando $N=20, n_{i}=3,5,8$ e 12, $\lambda_{1}=0,22$ e $\lambda_{2}=0,22 ; 0,70$ e 1,22. Observase que os resíduos condicional, marginal e aleatórios não seguem uma distribuição normal, enquanto o resíduo quantil aleatorizado segue uma distribuição aproximadamente normal.

As Figuras 6.9 a 6.12 apresentam gráficos de probabilidade normal dos resíduos quan- 
til aleatorizado, condicional, marginal e aleatórios para o modelo beta $t$-Student dado na equação (4.4) quando $N=20, n_{i}=3,5,8$ e $12, \nu_{1}=4$ e $\nu_{2}=3$ e 4 . O padrão observado nestas figuras é o mesmo que o padrão observado nas Figuras 6.1 e 6.4, os resíduos quantil aleatorizado e aleatórios seguem uma distribuição aproximadamente normal e os resíduos condicional e marginal não seguem uma distribuição normal mas provavelmente uma distribuição de cauda pesada.

As Figuras 6.13 a 6.16 exibem gráficos de probabilidade normal dos resíduos quantil aleatorizado, condicional, marginal e aleatórios para o modelo beta exponencial potência definido na equação (4.5) quando $N=20, n_{i}=3,5,8$ e $12, \lambda_{1}=0,44$ e $\lambda_{2}=0,44 ; 0,89$ e 1,33. O padrão observado nestas figuras é o mesmo observado nos casos dos modelos beta normal e beta $t$-Student.

\subsection{Conclusões}

O objetivo deste capítulo foi apresentar uma proposta de seleção de modelos de regressão beta com efeitos aleatórios para dados longitudinais e um conjunto de ferramentas de diagnóstico para estes modelos. Propomos um método de seleção de modelos apropriado para os modelos apresentados nos Capítulos 3, 4 e 5 no sentido de que as estruturas de regressão da média e da dispersão, os efeitos aleatórios e as estruturas de variância-covariância são selecionadas de forma algoritmica. Além disso, propomos vários resíduos para avaliar a adequação geral do modelo aos dados e identificar observações atípicas. É importante notar que para cada um dos resíduos propostos podemos construir gráficos de probabilidade meio normal para identificar observações ou indivíduos atípicos embora a distribuição empírica destes não seja a distribuição normal. Em todos os modelos beta considerados, independentemente da distribuição dos interceptos aleatórios, o resíduo quantil aleatorizado segue uma distribuição aproximadamente normal. Com exceção do modelo beta log-gama, o mesmo pode ser observado no caso dos resíduos aleatórios. Já os resíduos marginal e condicional seguem provavelmente distribuições de cauda mais pesadas que a normal. Assim, recomenda-se o uso do resíduo quantil aleatorizado. 

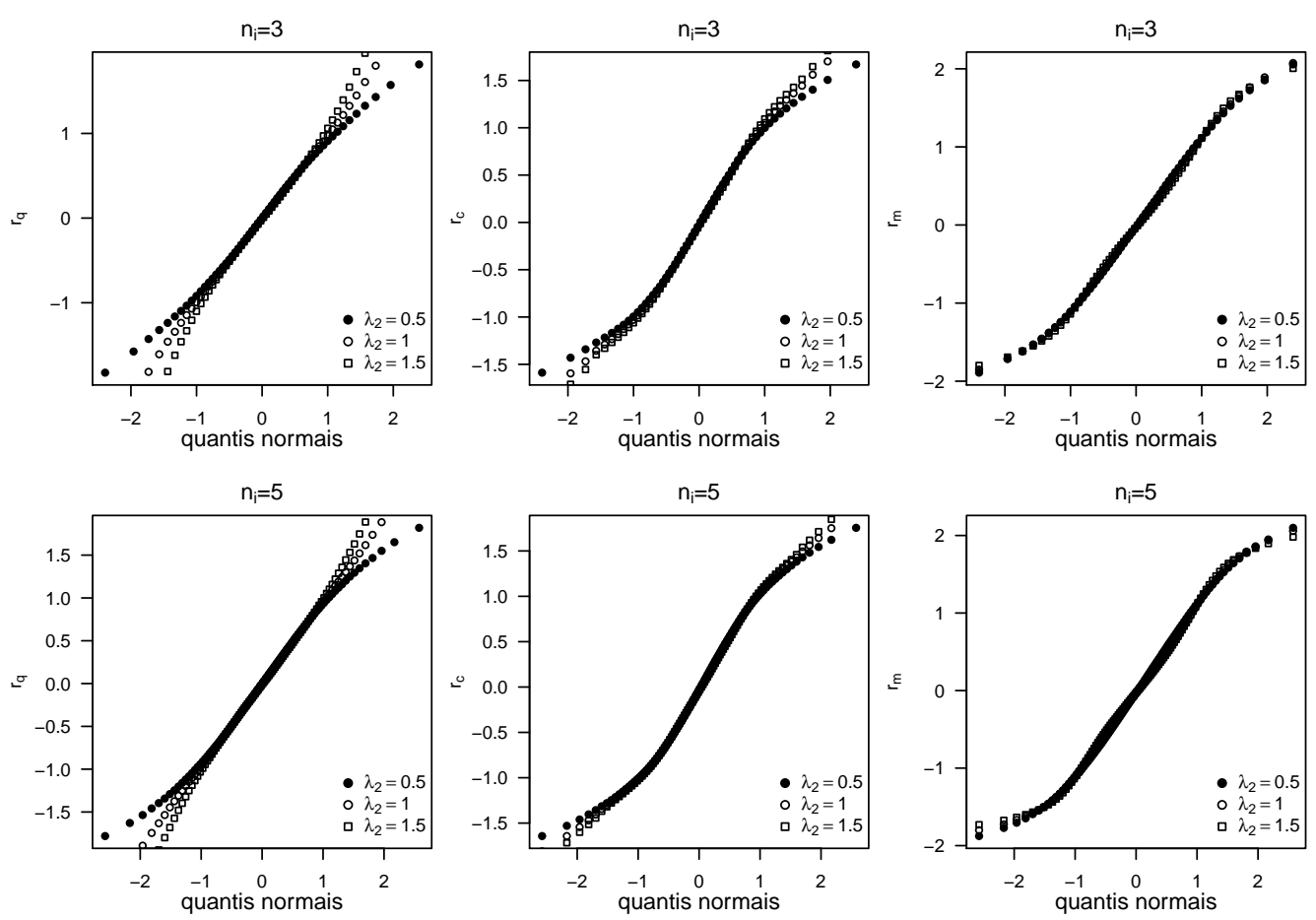

Figura 6.1: Gráficos de probabilidade normal dos resíduos quantil aleatorizado, condicional e marginal do modelo beta normal com $\lambda_{1}=0,5 ; N=20 ; n_{i}=3$ e $n_{i}=5$.
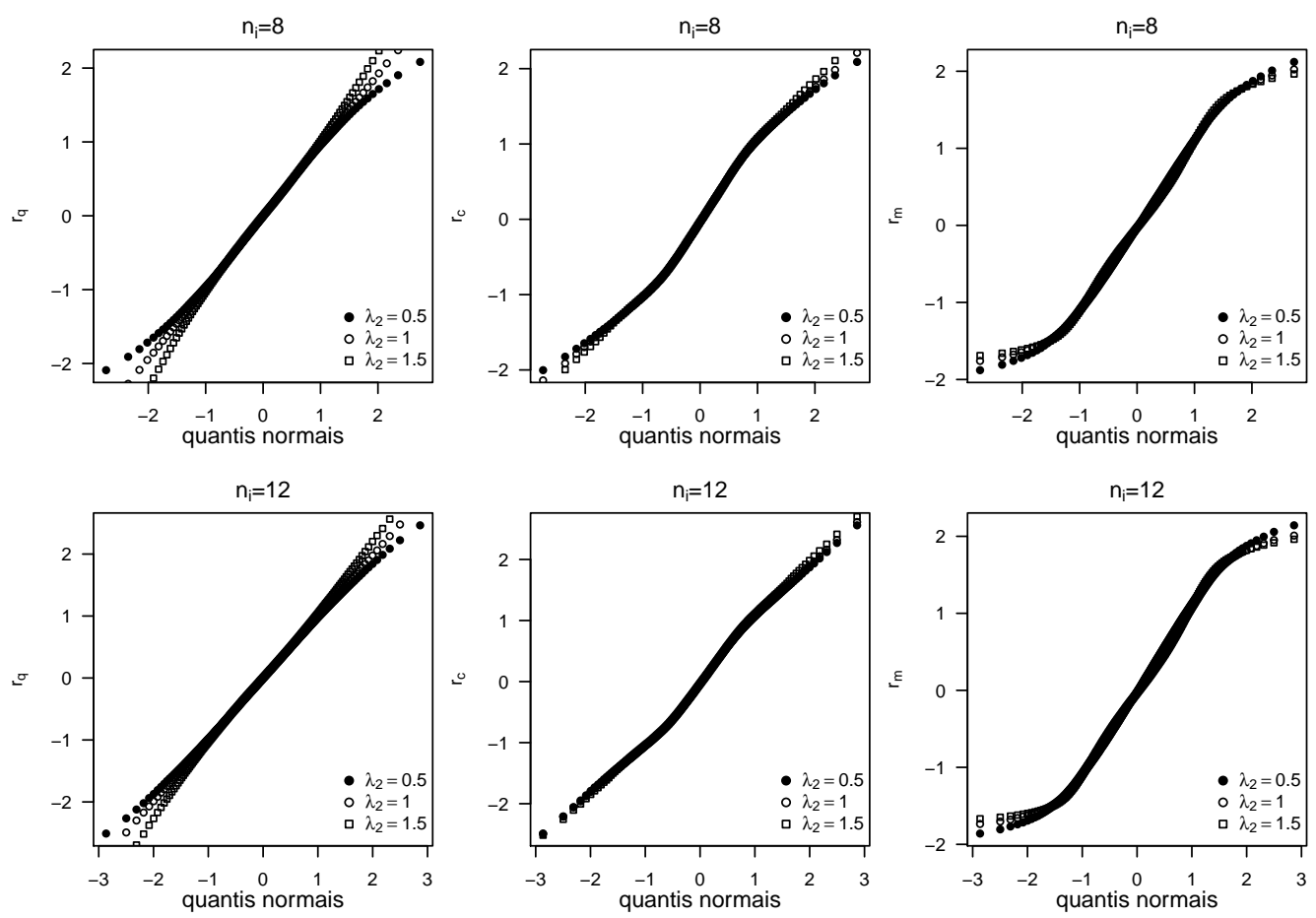

Figura 6.2: Gráficos de probabilidade normal dos resíduos quantil aleatorizado, condicional e marginal do modelo beta normal com $\lambda_{1}=0,5 ; N=20 ; n_{i}=8$ e $n_{i}=12$. 

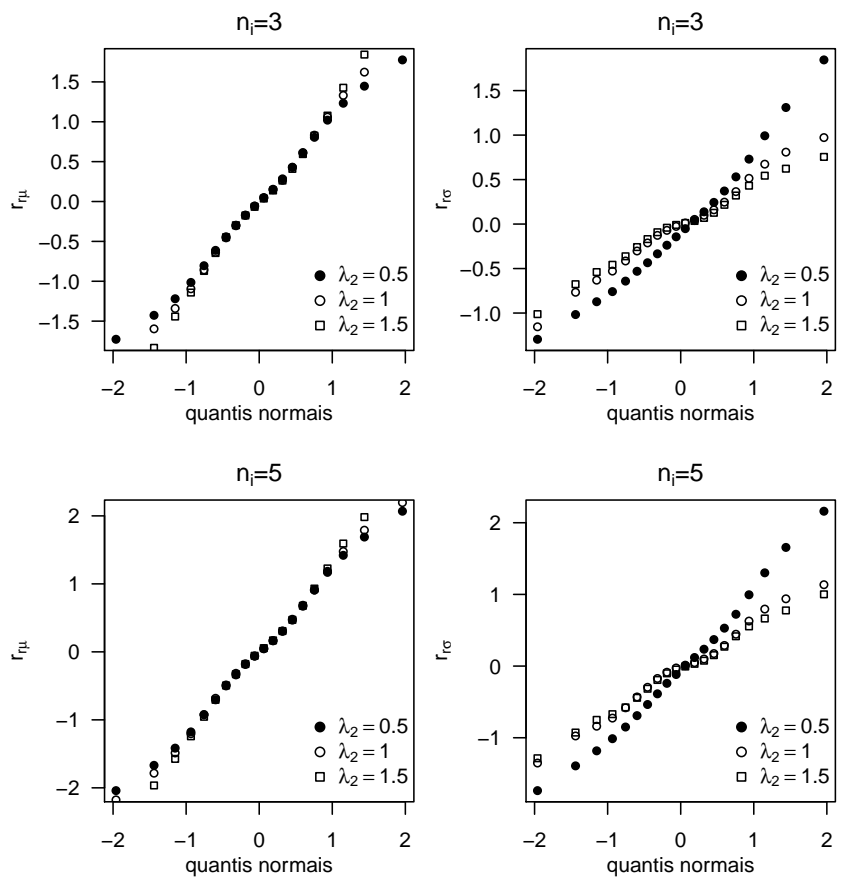

Figura 6.3: Gráficos de probabilidade normal dos resíduos aleatórios para $\mu$ e $\sigma$ do modelo beta normal com $\lambda_{1}=0,5 ; N=20 ; n_{i}=3$ e $n_{i}=5$.
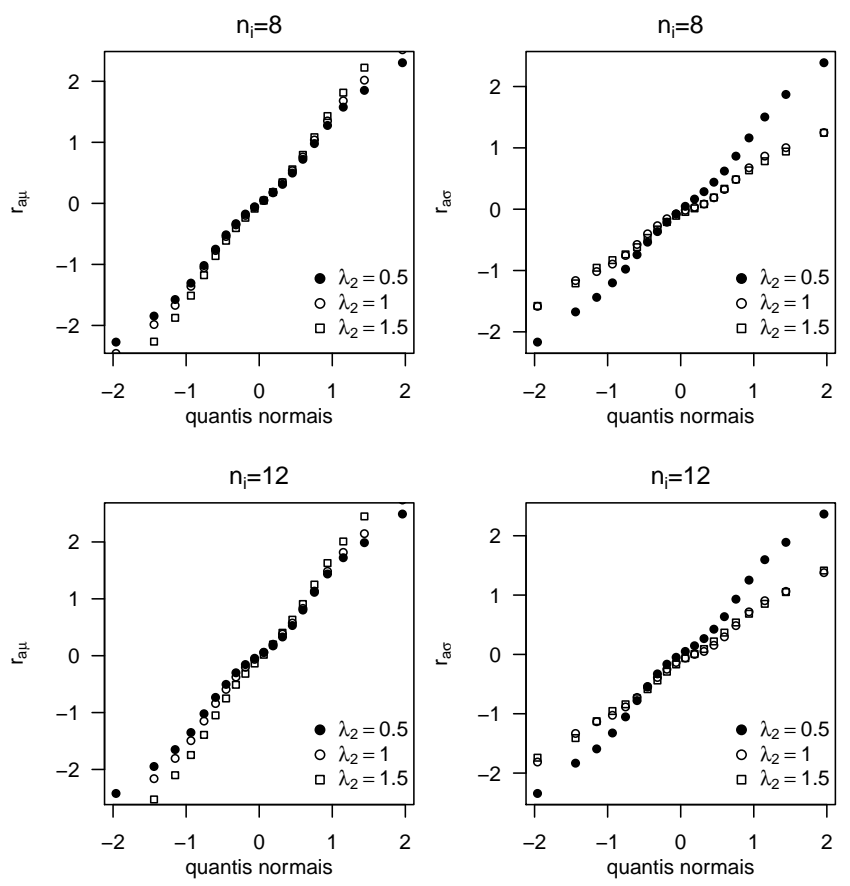

Figura 6.4: Gráficos de probabilidade normal dos resíduos aleatórios para $\mu$ e $\sigma$ do modelo beta normal com $\lambda_{1}=0,5 ; N=20 ; n_{i}=8$ e $n_{i}=12$. 

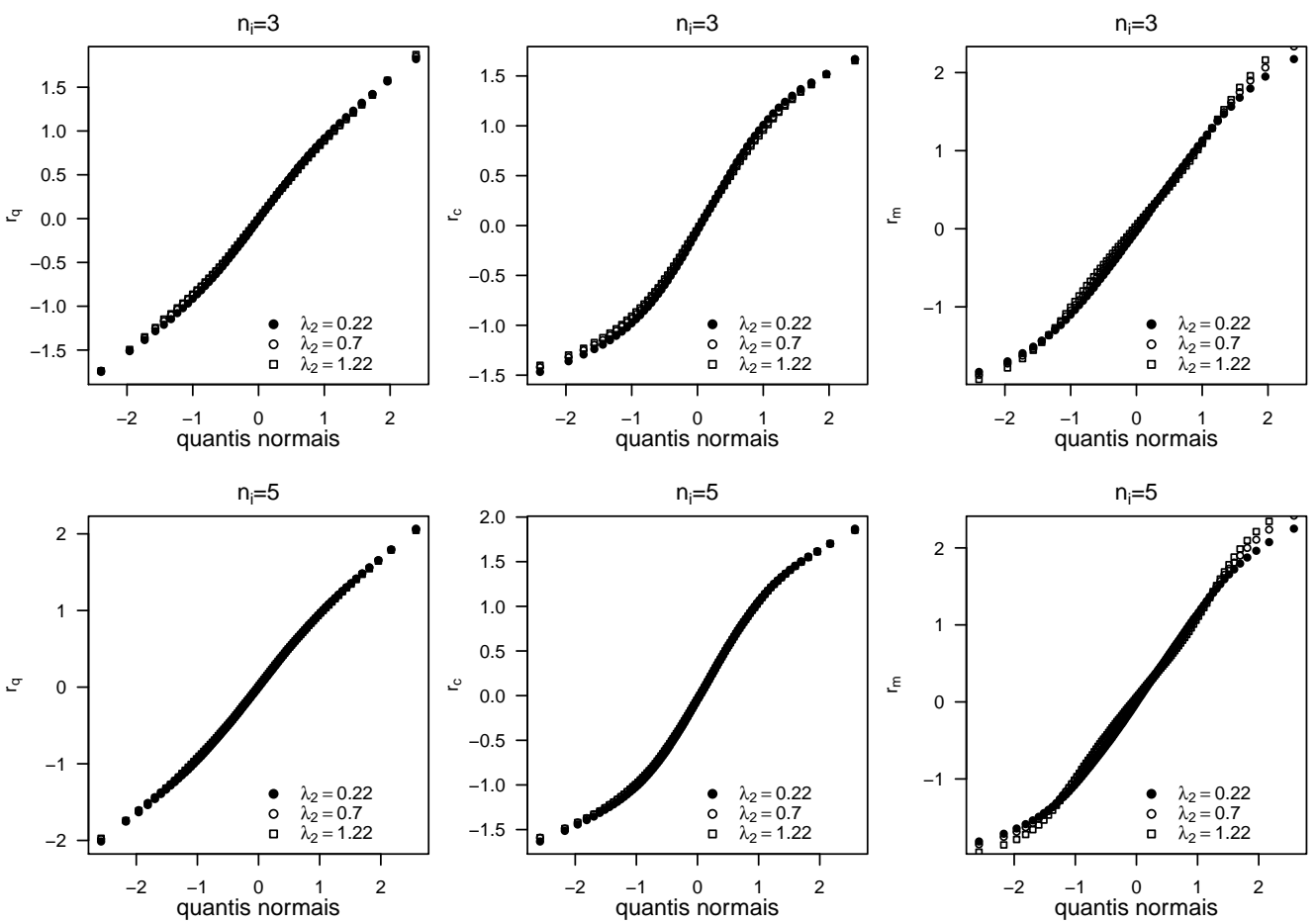

Figura 6.5: Gráficos de probabilidade normal dos resíduos quantil aleatorizado, condicional e marginal do modelo beta log-gama com $\lambda_{1}=0.22 ; N=20 ; n_{i}=3$ e $n_{i}=5$.
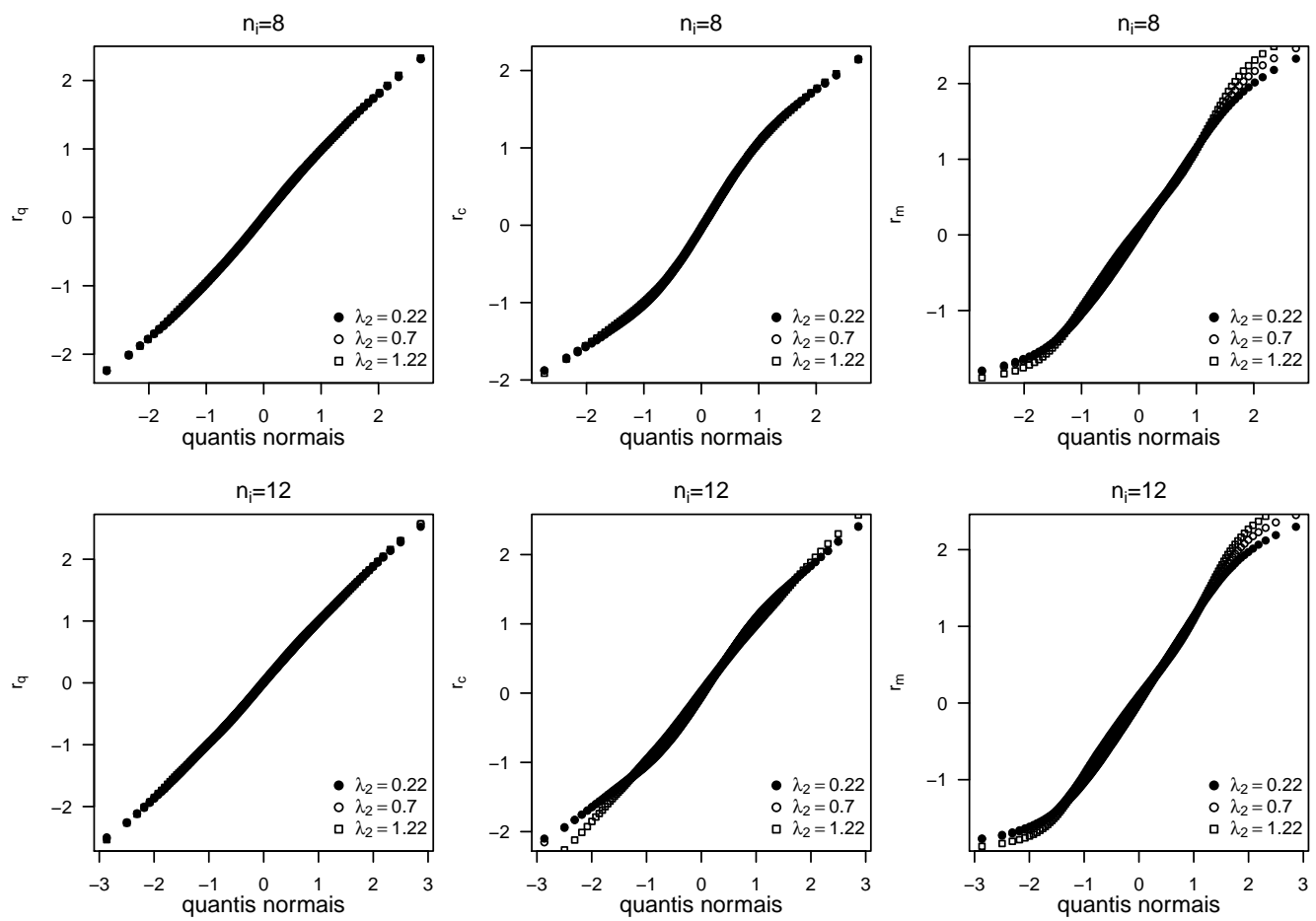

Figura 6.6: Gráficos de probabilidade normal dos resíduos quantil aleatorizado, condicional e marginal do modelo beta log-gama com $\lambda_{1}=0.22 ; N=20 ; n_{i}=8$ e $n_{i}=12$. 

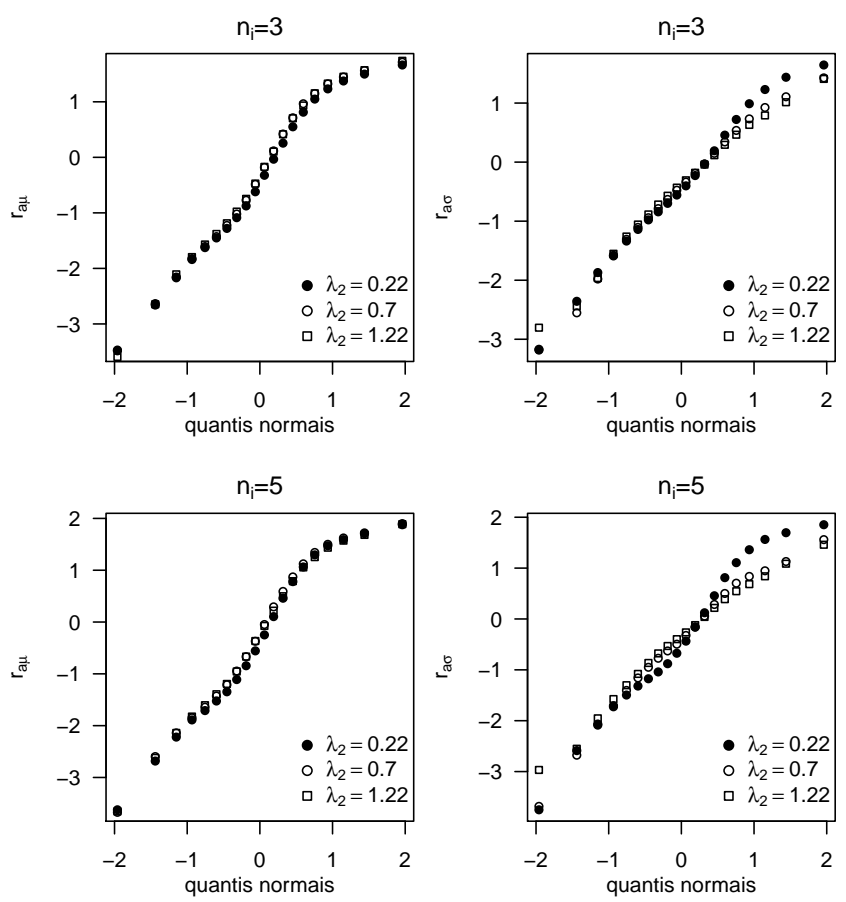

Figura 6.7: Gráficos de probabilidade normal dos resíduos aleatórios para $\mu$ e $\sigma$ do modelo beta log-gama com $\lambda_{1}=0.22 ; N=20 ; n_{i}=3$ e $n_{i}=5$.
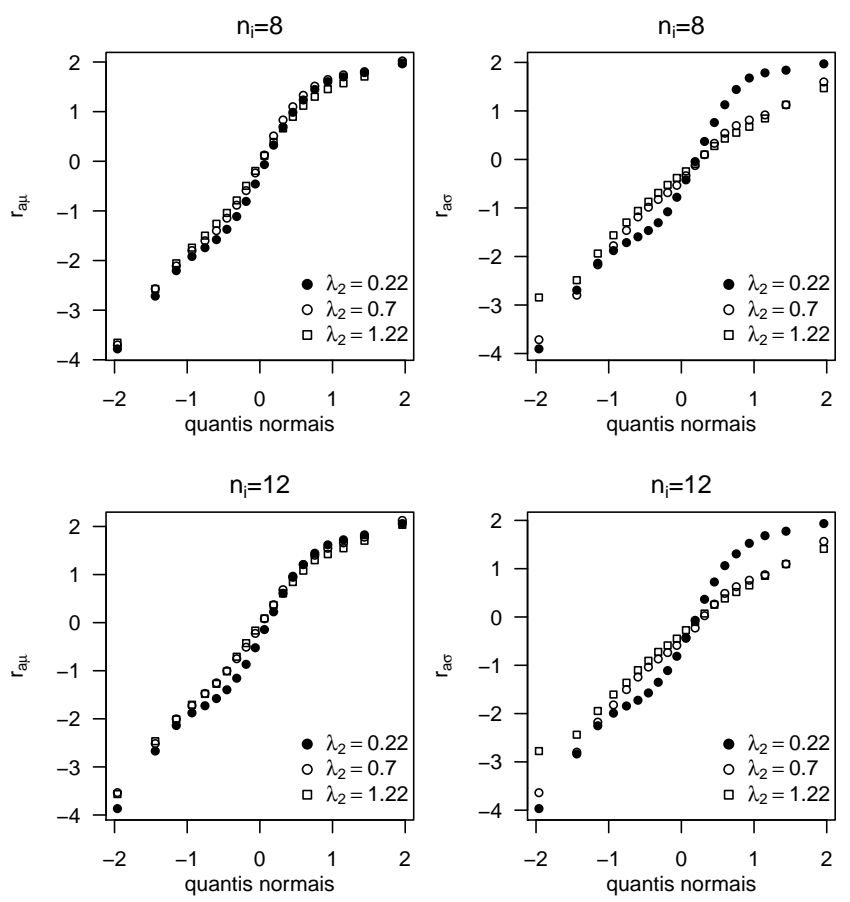

Figura 6.8: Gráficos de probabilidade normal dos resíduos aleatórios para $\mu$ e $\sigma$ do modelo beta log-gama com $\lambda_{1}=0.22 ; N=20 ; n_{i}=8$ e $n_{i}=12$. 

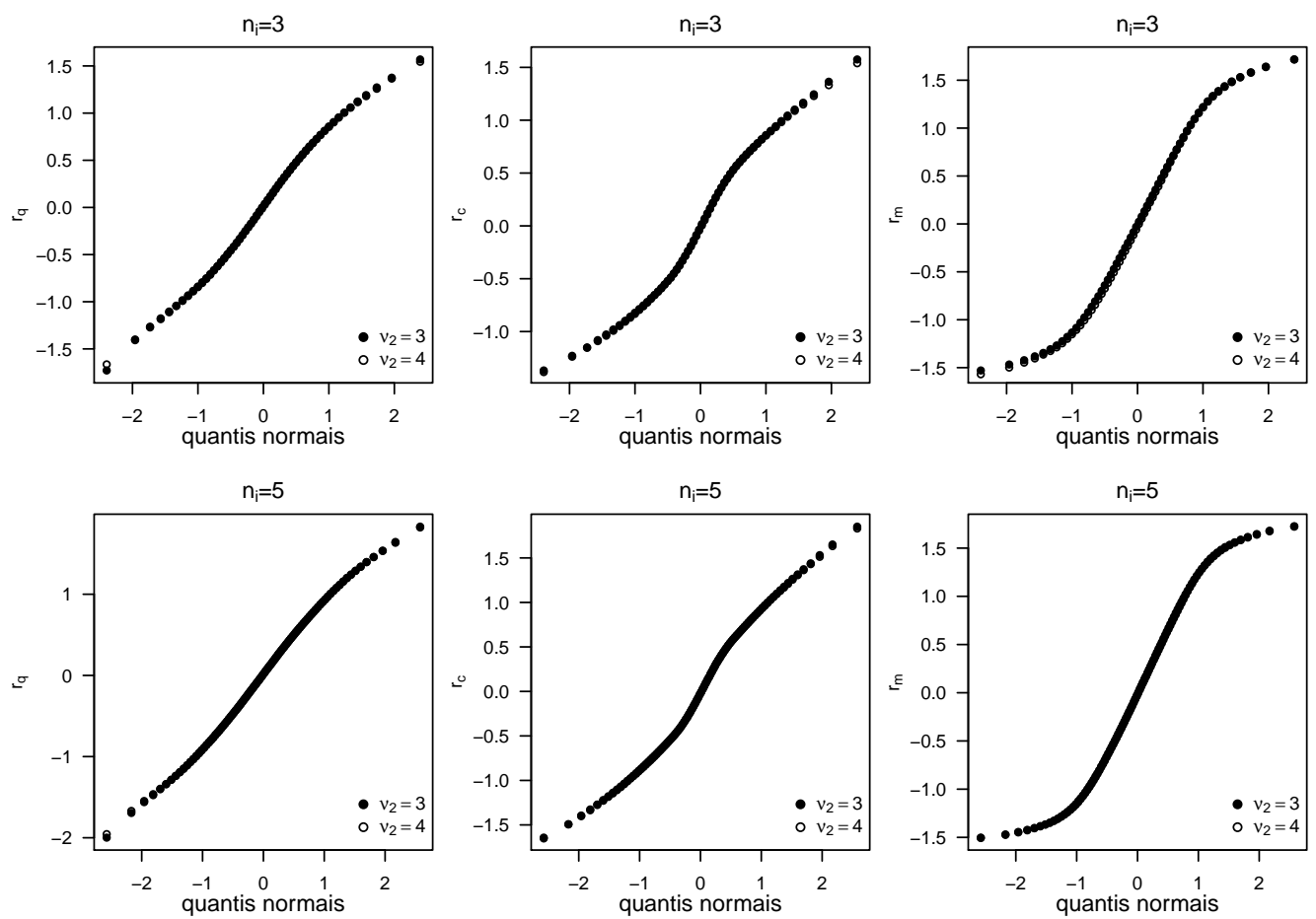

Figura 6.9: Gráficos de probabilidade normal dos resíduos quantil aleatorizado, condicional e marginal do modelo beta $t$-Student com $\nu_{1}=4 ; N=20 ; n_{i}=3$ e $n_{i}=5$.
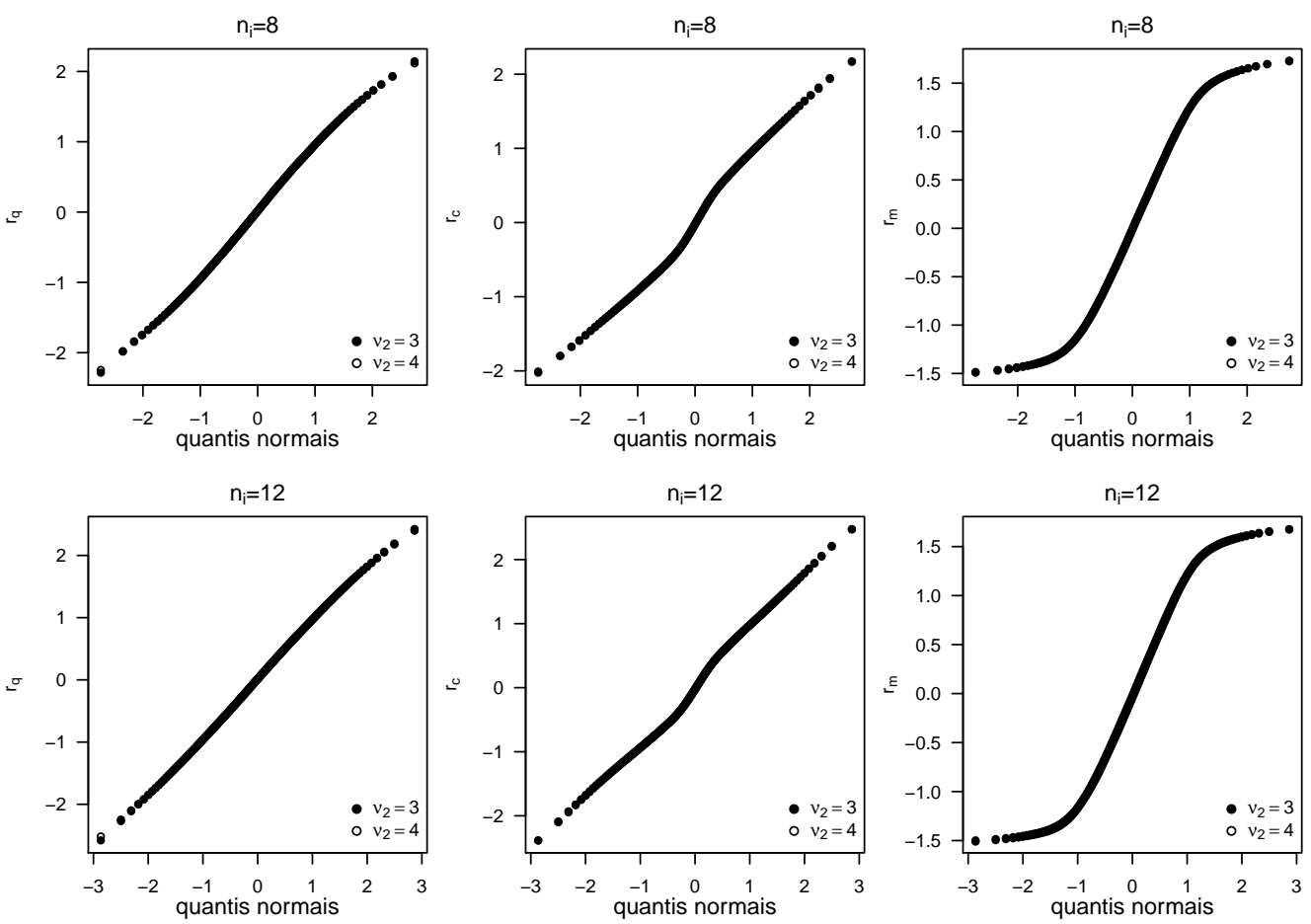

Figura 6.10: Gráficos de probabilidade normal dos resíduos quantil aleatorizado, condicional e marginal do modelo beta $t$-Student com $\nu_{1}=4 ; N=20 ; n_{i}=8$ e $n_{i}=12$. 

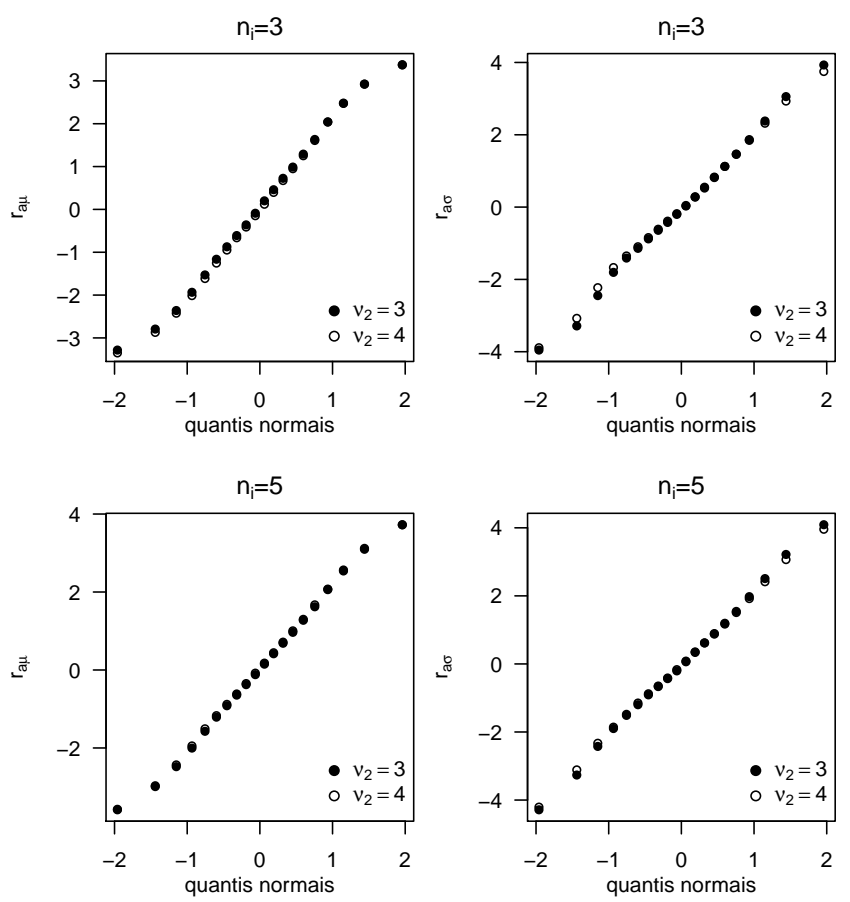

Figura 6.11: Gráficos de probabilidade normal dos resíduos aleatórios para $\mu$ e $\sigma$ do modelo beta t-Student com $\nu_{1}=4 ; N=20 ; n_{i}=3$ e $n_{i}=5$.
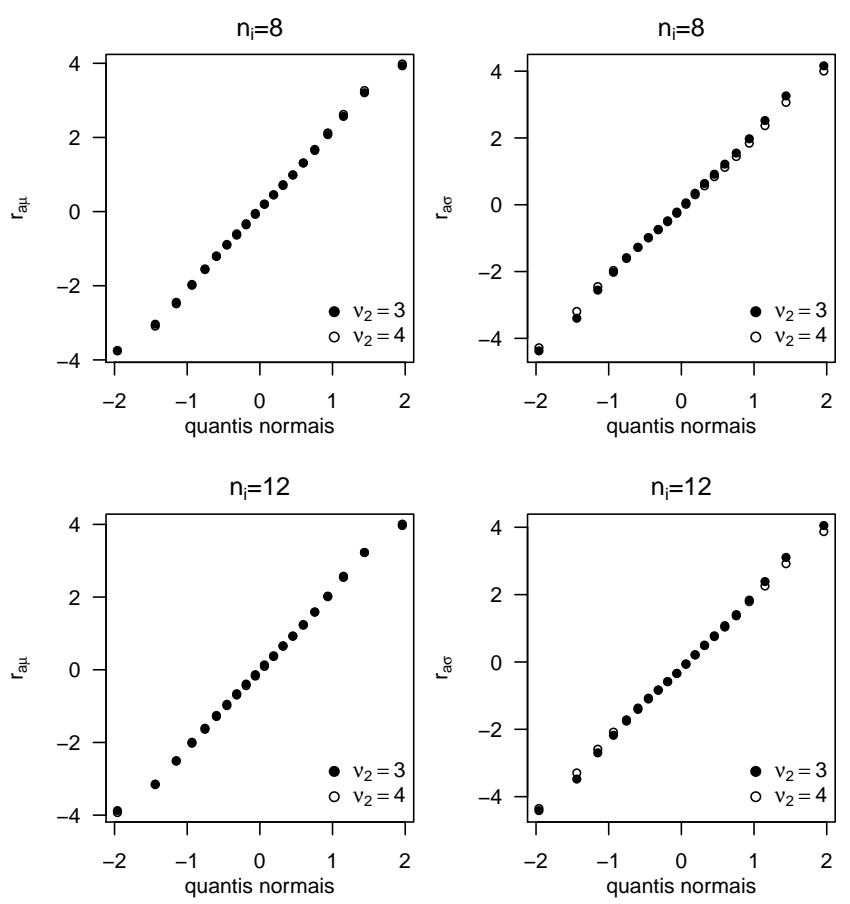

Figura 6.12: Gráficos de probabilidade normal dos resíduos aleatórios para $\mu$ e $\sigma$ do modelo beta $t$-Student com $\nu_{1}=4 ; N=20 ; n_{i}=8$ e $n_{i}=12$. 

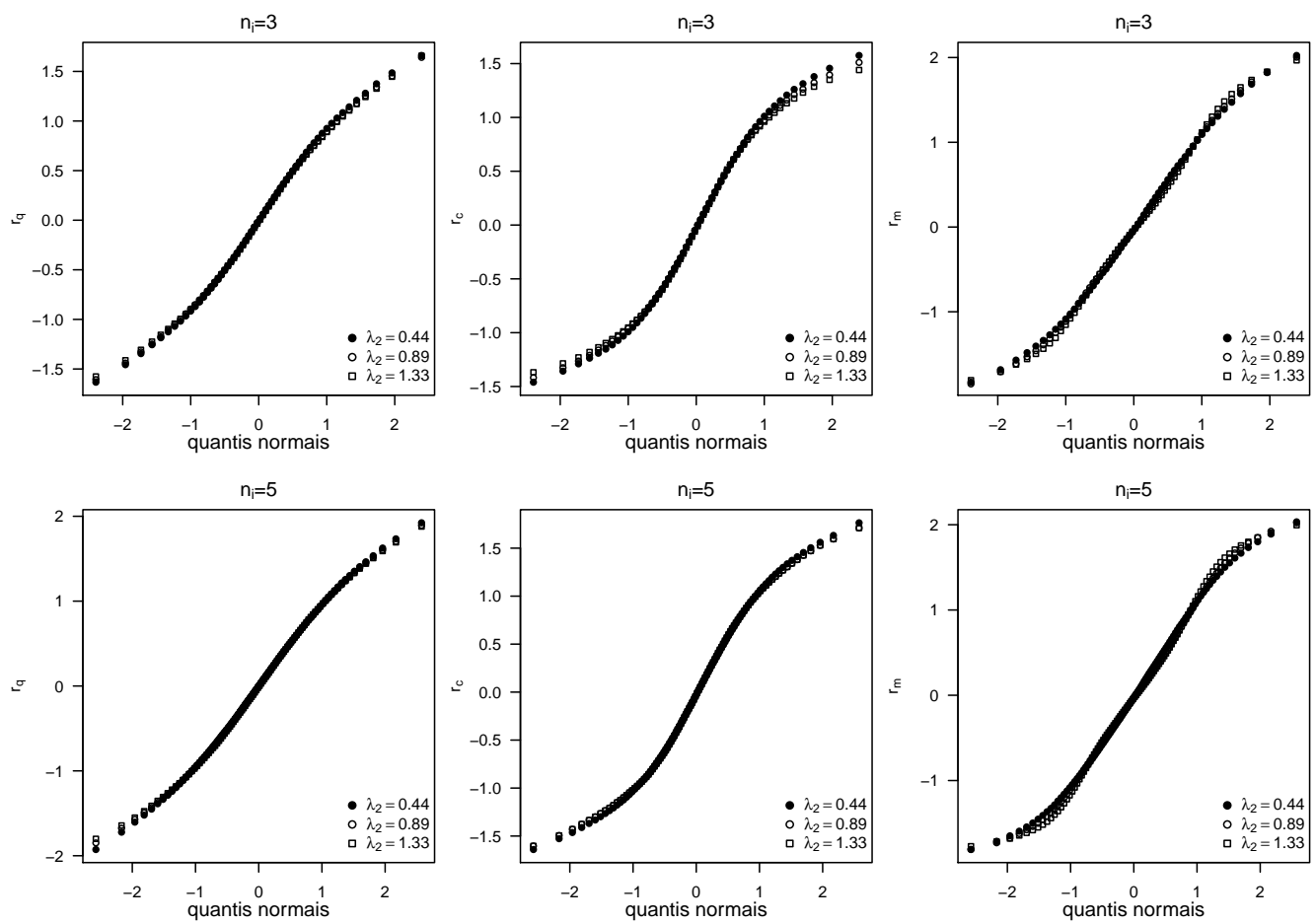

Figura 6.13: Gráficos de probabilidade normal dos resíduos quantil aleatorizado, condicional $e$ marginal do modelo beta exponencial potência com $\lambda_{1}=0.44 ; N=20 ; n_{i}=3$ e $n_{i}=5$.
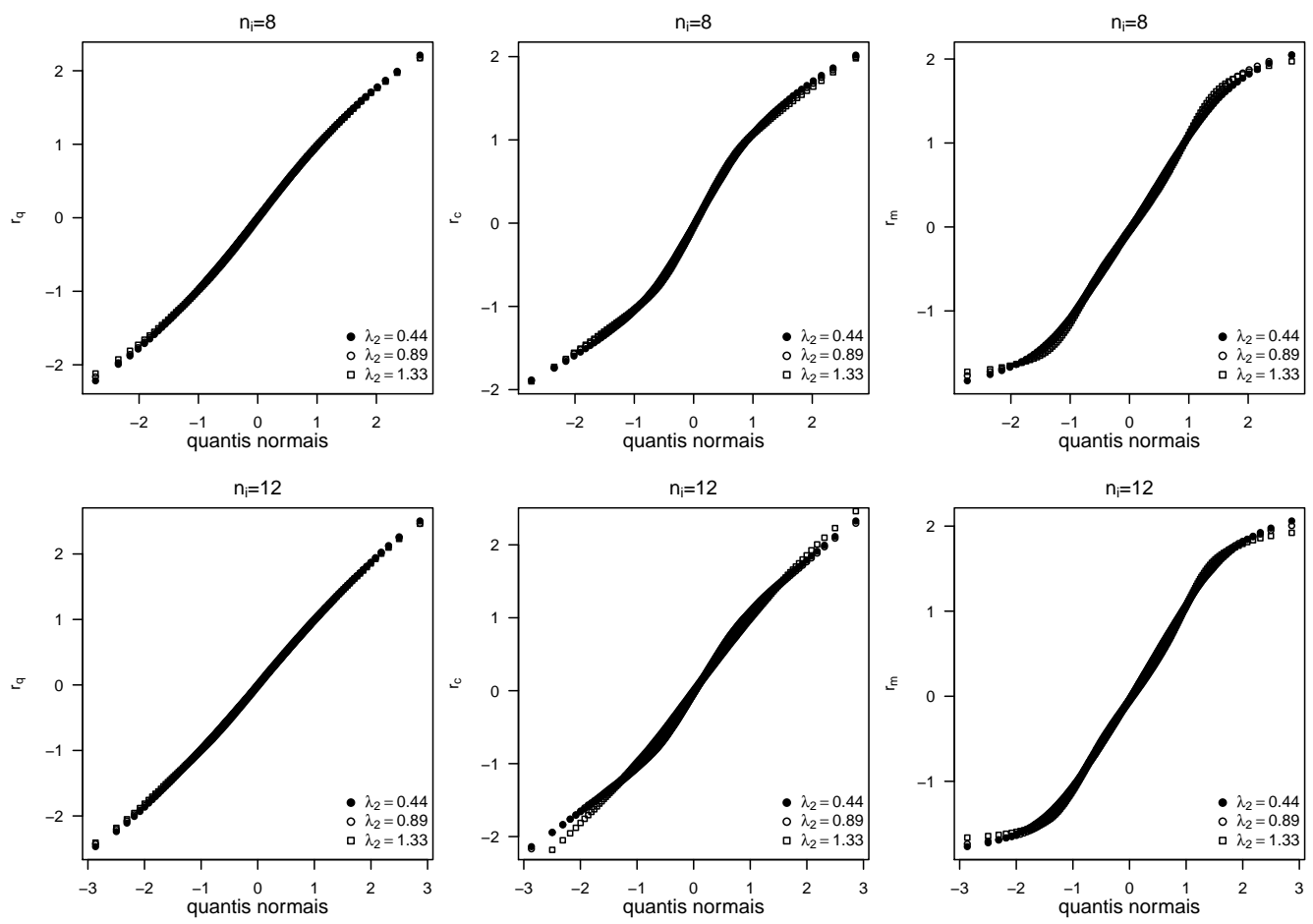

Figura 6.14: Gráficos de probabilidade normal dos resíduos quantil aleatorizado, condicional $e$ marginal do modelo beta exponencial potência com $\lambda_{1}=0.44 ; N=20 ; n_{i}=8$ e $n_{i}=12$. 

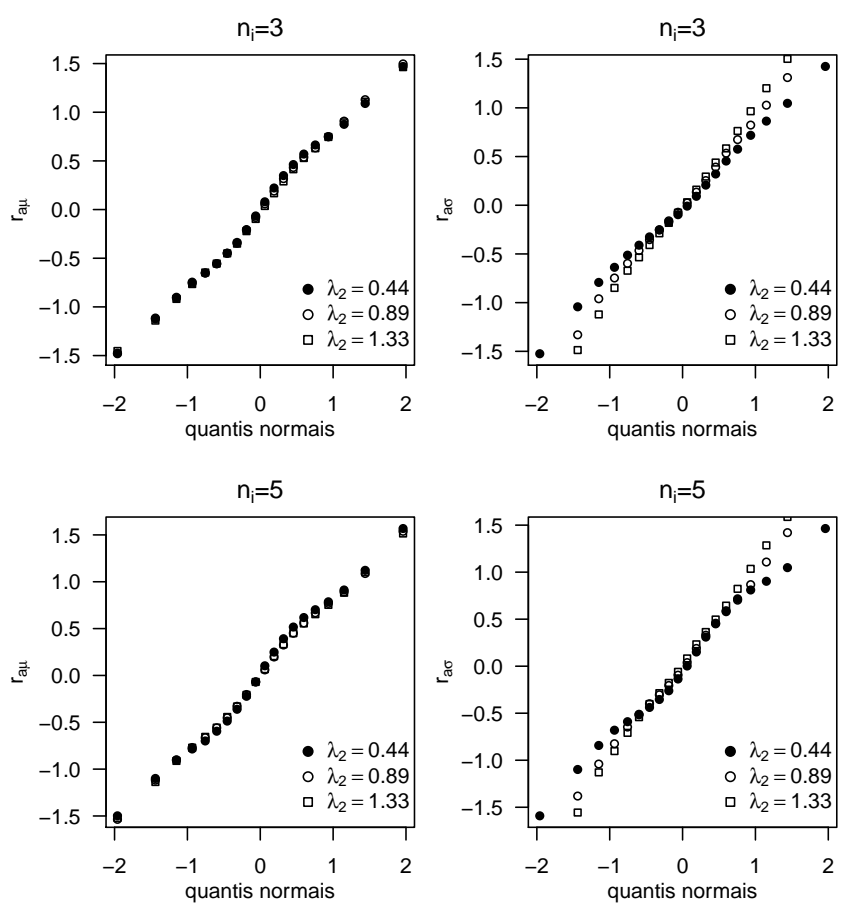

Figura 6.15: Gráficos de probabilidade normal dos resíduos aleatórios para $\mu$ e $\sigma$ do modelo beta exponencial potência com $\lambda_{1}=0.44 ; N=20 ; n_{i}=3$ e $n_{i}=5$.
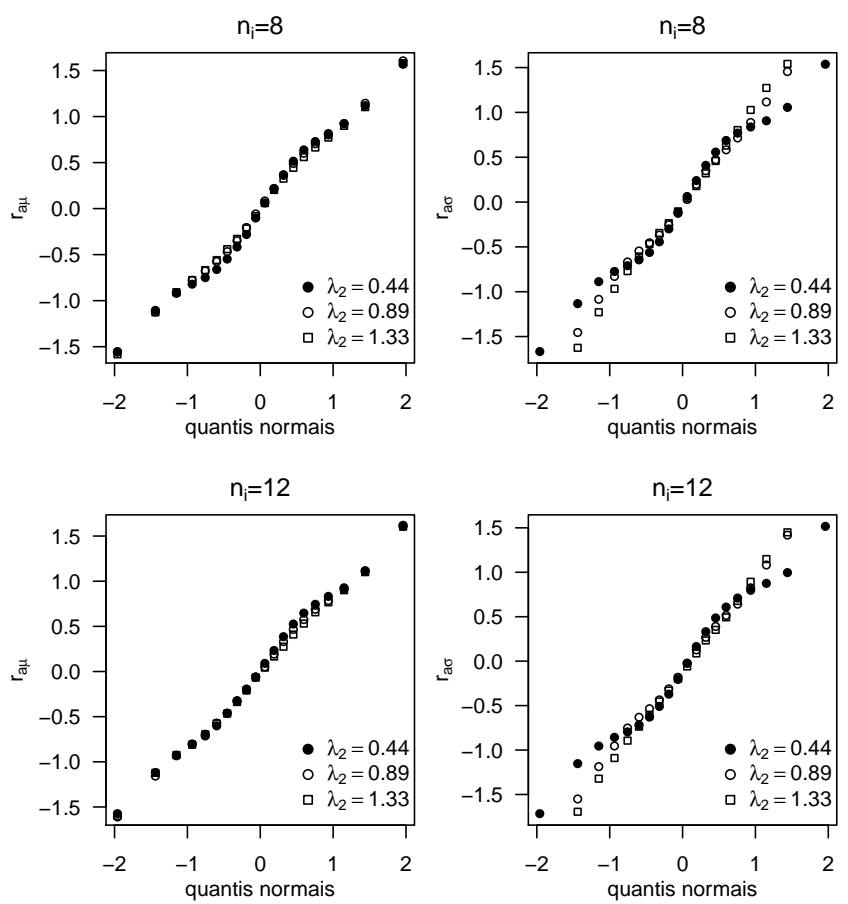

Figura 6.16: Gráficos de probabilidade normal dos resíduos aleatórios para $\mu$ e $\sigma$ do modelo beta exponencial potência com $\lambda_{1}=0.44 ; N=20 ; n_{i}=8$ e $n_{i}=12$. 


\section{Capítulo 7}

\section{Aplicações}

Neste capítulo ajustamos um modelo de regressão beta com intercepto aleatório a um conjunto de dados de um estudo prospectivo oftalmológico e um modelo de regressão beta com intercepto e inclinação aleatória a um conjunto de dados sobre as taxas de abandono escolar no estado de Antioquia na Colômbia. Os dois conjuntos de dados são utilizados para ilustrar a metodologia proposta incluindo a seleção de modelos e a análise de resíduos.

\subsection{Dados de estudo prospectivo oftalmológico}

Consideramos o conjunto de dados de um estudo prospectivo oftalmológico apresentado por Meyers et al. (1992) e previamente analisado por Song e Tan (2000), Song et al. (2004), Song (2007), Zhang e Wei (2008), Qiu et al. (2008), Venezuela (2008) e Wei e Zhang (2009). Os dados são descritos em detalhe no material suplementar do livro Correlated Data Analysis: Modeling, Analytics, and applications de Song (2007). Os dados são longitudinais e foram coletados em um estudo oftalmológico que avalia o uso de gás intraocular $\left(C_{3} F_{8}\right)$ em reparação cirúrgica da retina. A variável resposta foi a porcentagem de volume de gás ainda presente nos olhos com relação ao volume de gás injetado no momento da cirurgia e as variáveis explicativas de interesse foram o tempo após a injeção do gás e a concentração do gás utilizado $(15 \%, 20 \%$ e 25\%). A variável resposta tem zeros e uns. Nesta aplicação, usamos as observações de 29 pacientes que não tem esses valores.

No momento da cirurgia, um determinado volume de gás intraocular foi injetado nos olhos dos pacientes. Os pacientes foram observados de 3 a 15 vezes num período de três meses, e em cada visita, foi registrada a porcentagem do volume de gás. As visitas para cada paciente não ocorreram regularmente de modo que as medidas foram coletadas em momentos espaçados de forma desigual. O objetivo deste estudo foi avaliar a cinética por meio da taxa de decaimento do desaparecimento do gás através dos três níveis de concentração utilizados.

A Figura 7.1 apresenta os gráficos de perfis dos três subconjuntos de dados correspon- 
dentes a cada uma das três concentrações. Em geral, uma tendência decrescente no tempo é observada nos gráficos. O histograma de frequências e o boxplot apresentados na Figura 7.2 permitem concluir que os dados são assimétricos. A porcentagem do volume de gás mínima, máxima e média é de 0.05, 0.99 e 0.60.

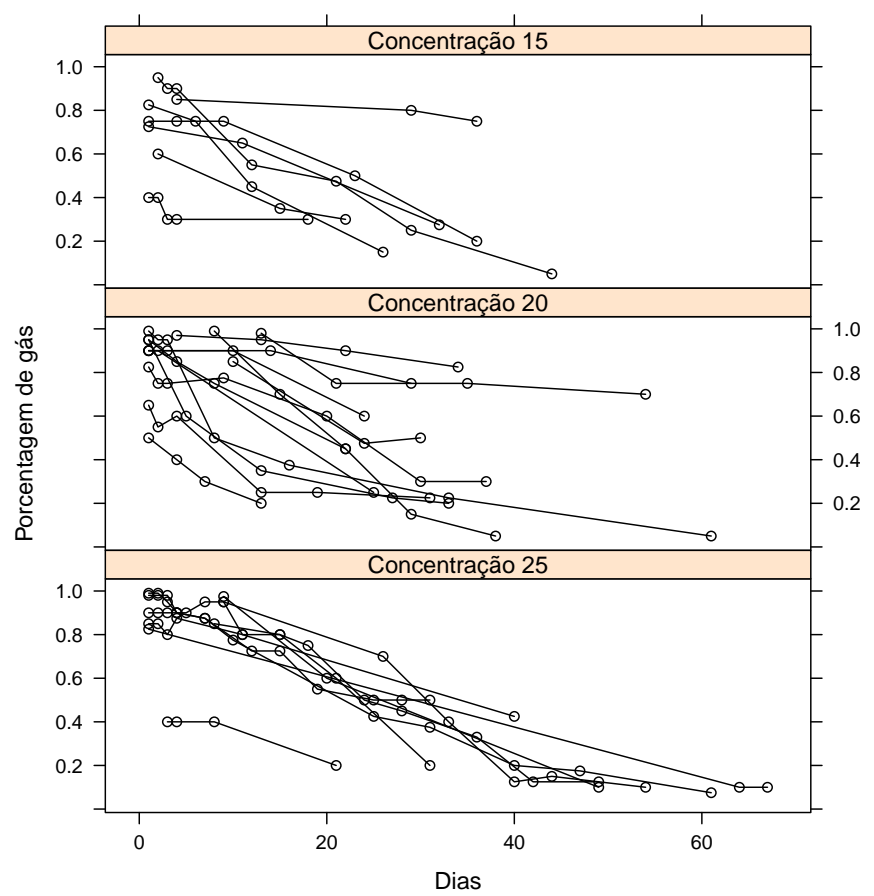

Figura 7.1: Gráfico de perfis da porcentagem observada de volume de gás presente nos olhos dos pacientes segundo os níveis de concentração do gás.

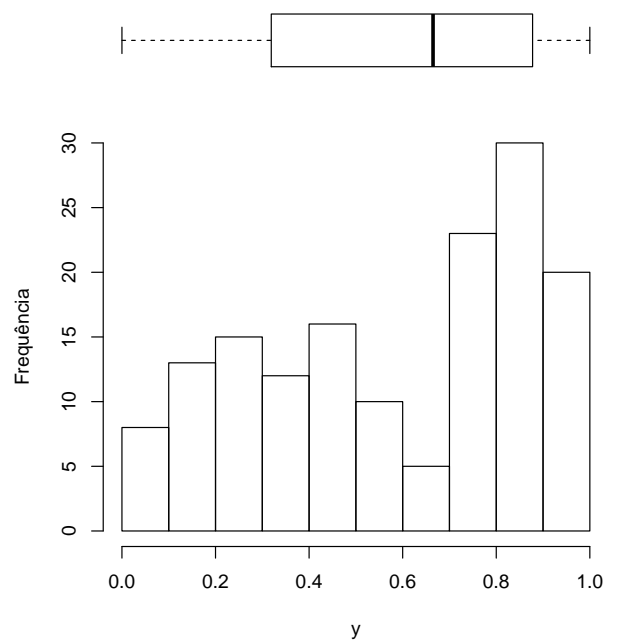

Figura 7.2: Histograma de frequências e boxplot da pocentagem observada de volume de gás presente nos olhos dos pacientes. 
Seja $y_{i j}$ a porcentagem de volume de gás para o $i$-ésimo paciente no tempo $t_{i j}$, com $i=1, \ldots, 29$ e $j=1, \ldots, n_{i}$. Consideramos o modelo de regressão beta com intercepto aleatório normal dado por

$$
\begin{aligned}
y_{i j} \mid \gamma_{i 1}, \gamma_{i 2} & \stackrel{\text { ind }}{\sim} \operatorname{Be}\left(\mu_{i j}, \sigma_{i j}\right), \\
\gamma_{i 1} & \stackrel{\text { i.i.d }}{\sim} N\left(0, \lambda_{1}^{2}\right), \\
\gamma_{i 2} & \stackrel{\text { i.i.j. }}{\sim} N\left(0, \lambda_{2}^{2}\right),
\end{aligned}
$$

com

$$
\begin{aligned}
& \operatorname{logito}\left(\mu_{i j}\right)=\beta_{11}+\beta_{21} \log \left(t_{i j}\right)+\beta_{31} \log ^{2}\left(t_{i j}\right)+\beta_{41} x_{i j}+\gamma_{i 1}, \\
& \operatorname{logito}\left(\sigma_{i j}\right)=\beta_{12}+\beta_{22} \log \left(t_{i j}\right)+\beta_{32} \log ^{2}\left(t_{i j}\right)+\beta_{42} x_{i j}+\gamma_{i 2},
\end{aligned}
$$

em que $t_{i j}$ é o tempo após a cirurgia e $x_{i j}$ é a concentração padrão do gás definida como

$$
x_{i j}=\frac{\text { Concentrao }_{i j}-20}{5}=\left\{\begin{aligned}
-1 & \text { se a concentração de gás é } 15 \\
0 & \text { se a concentração de gás é } 20 \\
1 & \text { se a concentração de gás é } 25 .
\end{aligned}\right.
$$

A estrutura de regressão para a média é baseada no modelo de regressão simplex para os dados do estudo prospectivo oftalmológico proposto por Song e Tan (2000), Song (2007), Zhang e Wei (2008), Qiu et al. (2008), Venezuela (2008) e Wei e Zhang (2009). O critério de seleção do modelo é o menor valor do critério de informação de Akaike, AIC. O modelo selecionado que foi ajustado aos dados foi o seguinte

$$
\begin{aligned}
& \operatorname{logito}\left(\mu_{i j}\right)=\beta_{11}+\beta_{31} \log ^{2}\left(t_{i j}\right)+\beta_{41} x_{i j}+\gamma_{i 1}, \\
& \operatorname{logito}\left(\sigma_{i j}\right)=\beta_{12}+\gamma_{i 2} .
\end{aligned}
$$

As estimativas de máxima verossimilhança dos parâmetros do modelo proposto foram obtidas segundo a metodologia apresentada no Capítulo 3 com 21 pontos de quadratura. Escolhimos esse número de pontos de quadratura porque o aumento de pontos contribuiu pouco à aproximação do logaritmo da função de verossimilhança, isto é, as estimativas se mantiveram no mesmo valor à medida que aumentamos o número de pontos de quadratura. As estimativas apresentadas na Tabela 7.1 indicam que a concentração padrão do gás e $\log ^{2}\left(t_{i j}\right)$ são estatisticamente significativas o que indica que a porcentagem média de volume de gás presente nos olhos diminui com o passar do tempo, por outro lado aumenta com uma maior concentração do gás. Além disso, nota-se que a estimativa de $\lambda_{1}$ é maior do que a de $\lambda_{2}$, ou seja, há uma maior variabilidade no intercepto aleatório da média que na variância. As estimativas de máxima verossimilhança dos parâmetros da estrutura de regressão da média e as conclusões do modelo são semelhantes às apresentadas no trabalho de Qiu et al. (2008) 
de modelos de regressão simplex mistos.

Tabela 7.1: Estimativas, erros padrões e valores-p do modelo beta normal ajustado aos dados da porcentagem de volume de gás presente nos olhos.

\begin{tabular}{ccccc}
\hline & Parâmetro & Estimativa & ep & valor-p \\
\hline$\mu$ & $\beta_{11}$ & 1,673 & 0,120 & $<2,00 \mathrm{e}-16$ \\
& $\beta_{31}$ & $-0,262$ & 0,015 & $<2,00 \mathrm{e}-16$ \\
& $\beta_{41}$ & 0,314 & 0,080 & $9,31 \mathrm{e}-05$ \\
$\sigma$ & $\beta_{12}$ & $-1,166$ & 0,119 & $<2,00 \mathrm{e}-16$ \\
\hline$\mu$ & $\lambda_{1}$ & 1,109 & 0,093 & \\
$\sigma$ & $\lambda_{2}$ & 0,322 & 0,156 & \\
\hline
\end{tabular}

Os gráficos de probabilidade meio-normal com envelope simulado do resíduo quantil aleatorizado, condicional e marginal são apresentados na Figura 7.3. Nota-se que nenhuma observação fica fora do envelope simulado e portanto pode-se concluir que o modelo de regressão beta com intercepto aleatório normal ajusta-se adequadamente aos dados.
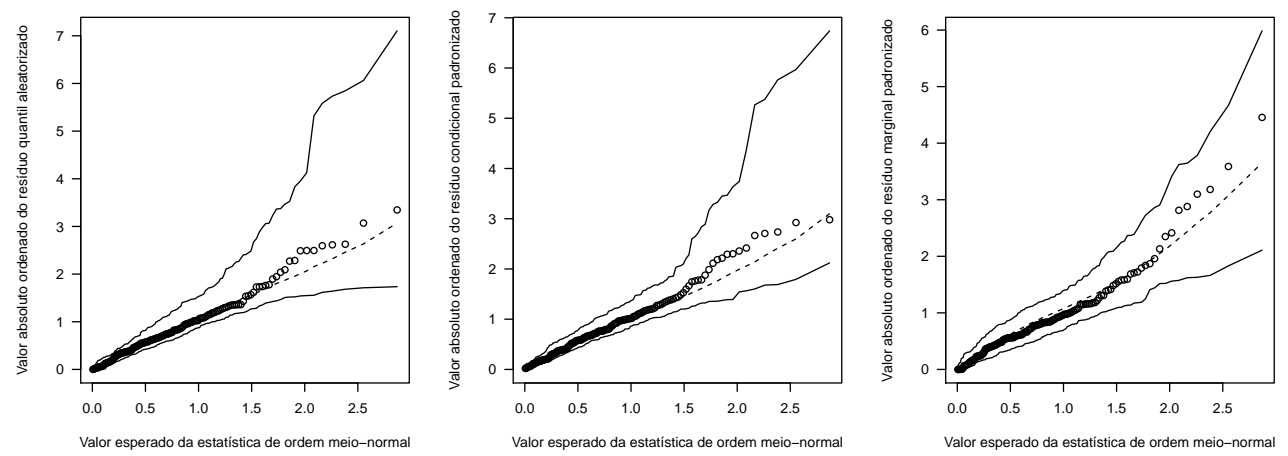

Figura 7.3: Gráficos de probabilidade meio-normal com envelope simulado do resíduo quantil aleatorizado, condicional e marginal do modelo beta normal ajustado aos dados da porcentagem de volume de gás presente nos olhos dos pacientes.

Para ilustrar, a Figura 7.4 apresenta os perfis individuais da porcentagem de volume de gás de alguns pacientes selecionados arbitrariamente, 1,2,17,18, 22 e 31. As linhas sobrepostas denotam o ajuste do modelo de regressão beta. Nota-se que estas linhas seguem o comportamento dos dados originais, indicando o bom ajuste do modelo ajustado.

\subsection{Dados de taxa de abandono escolar}

O conjunto de dados corresponde à taxa de abandono escolar no ensino fundamental $\left(6^{\circ}\right.$ a $9^{\circ}$ ) e no ensino médio $\left(10^{\circ}\right.$ a $\left.11^{\circ}\right)$ das escolas públicas dos municípios do estado de Antioquia, Colômbia, nos anos 2002, 2004, 2006 e 2008. Os dados são de uso público e podem ser consultados no Anuário estatístico de Antioquia no website do Departamento administrativo de planejamento do Departamento de Antioquia, http://antioquia.gov.co/index.php/planeacion/6865-antioquia-estadisticas-e-indicadores. A taxa de abandono escolar é uma taxa anual que 


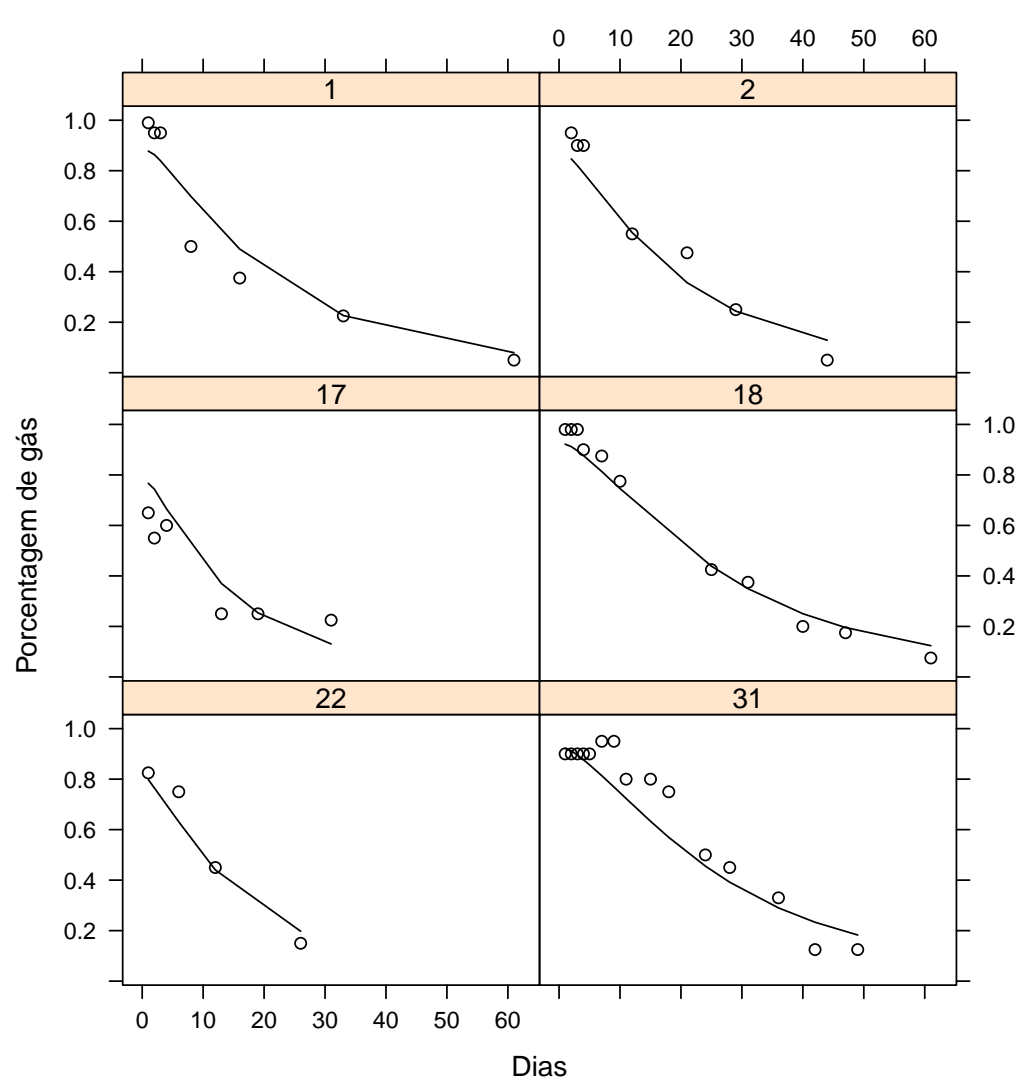

Figura 7.4: Dados observados e predição da porcentagem de volume de gás presente nos olhos nos pacientes 1,2,17,18,22 e 31 .

é definida como a proporção de alunos que abandonam um dado ano escolar, tomando como referência o total de alunos matriculados nesse ano de escolaridade.

A variável resposta y é a taxa de abandono escolar no ensino fundamental e médio em escolas públicas. As variáveis explicativas em relação às taxas de abandono nos municípios são: a porcentagem da população em domicílios com necessidades básicas insatisfeitas $\left(x_{1}\right)$, o número de professores em escolas públicas $\left(x_{2}\right)$, o número de escolas públicas $\left(x_{3}\right)$ e a região do estado de Antioquia $\left(x_{4}\right)$ ( SudOeste (SO), Leste (L), NorDeste (ND), Norte (N), Oeste (O), Bajo Cauca (BC), Magdalena Medio (MM), Uraba (U) e Valle de Aburra (VA)).

Consideramos 61 municípios que têm informações em relação à variável resposta e as variáveis explicativas nos quatro anos. Cada uma das regiões está representada por un número especifico de municípios, a região SO está representada por municípios, a região por municípios, a região ND por municípios, a região N por municípios, a região O por municípios, a região $\mathrm{BC}$ por municípios, a região $\mathrm{MM}$ por municípios, a região $\mathrm{U}$ por municípios e a região VA por municípios. O objetivo do estudo é investigar o efeito de fatores socieconômicos e de acessibilidade a educação na taxa de abandono escolar no ensino fundamental e médio. 
A Figura 7.5 apresenta os gráficos de perfis da taxa de abandono escolar no ensino fundamental e médio dos municípios segundo a região do estado a que pertencem. Nota-se que os municípios do BC apresentam as maiores taxas de abandono e os municípios do VA apresentam as menores taxas de abandono. A Figura 7.6 mostra o histograma de frequências e o boxplot dos dados observados. Nota-se que a distribuição da taxa de abandono escolar é asimétrica positiva. Os valores mínimo e máximo são $0.03 \%$ e $16.18 \%$, respectivamente.

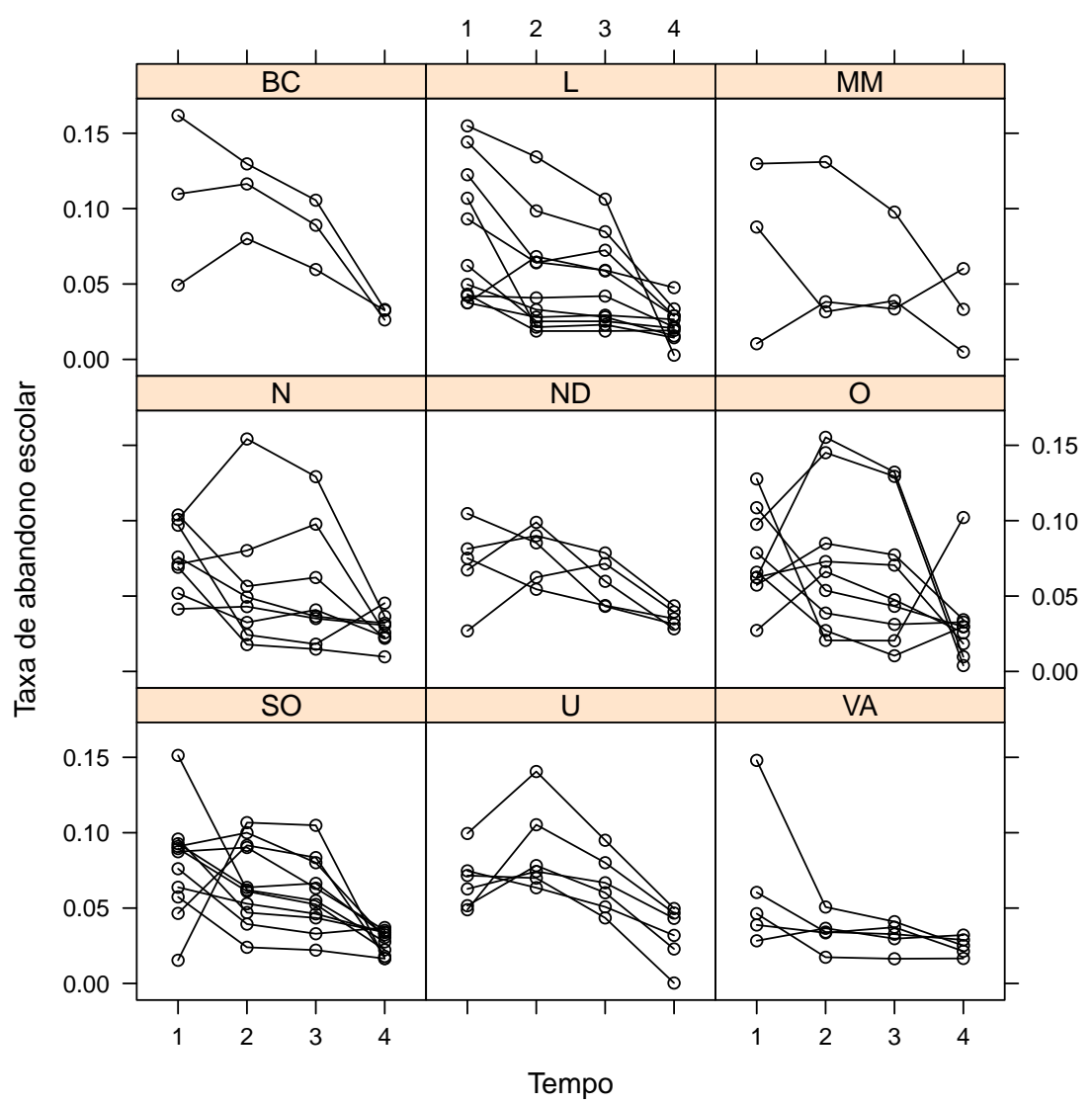

Figura 7.5: Gráfico de perfis da taxa observada de abandono escolar no ensino fundamental e médio dos municípios segundo a região do estado de Antioquia.

Seguindo a metodologia apresentada no Capítulo 6 para selecionar a estrutura de regressão e os termos preditores dos modelos de regressão de $\mu$ e $\sigma$ foram considerados diferentes modelos. As Tabelas 7.2 e 7.3 mostram os modelos e os respectivos valores do AIC. Em negrito se destacam os valores menores do critério AIC obtidos em cada passo da seleção dos efeitos fixos e aleatórios. 

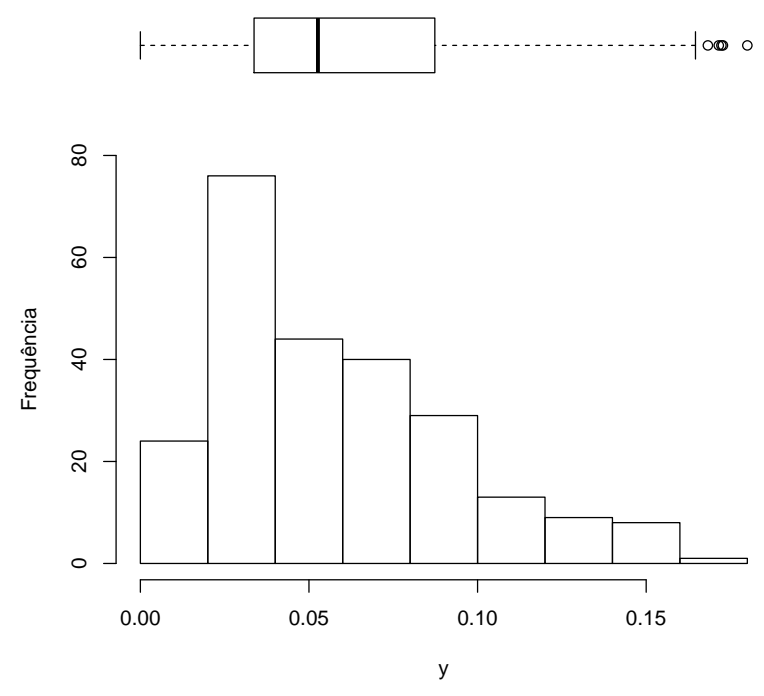

Figura 7.6: Histograma de frequências e boxplot da taxa observada de abandono escolar no ensino fundamental e médio nos municípios.

Tabela 7.2: Modelos beta normal ajustados à estrutura de regressão de $\mu$ e valores AIC.

\begin{tabular}{llc}
\hline Passos & Modelo & AIC \\
\hline 1 & $\beta_{11}+\gamma_{i 11}$ & $\mathbf{- 9 8 5 . 0 9 1}$ \\
\hline 2 & $\beta_{11}+\gamma_{i 11}+\beta_{21} t_{i j}$ & $\mathbf{- 1 0 7 2 . 5 7}$ \\
& $\beta_{11}+\gamma_{i 11}+\beta_{21} t_{i j}+\beta_{31} t_{i j}^{2}$ & -1068.24 \\
& $\beta_{11}+\gamma_{i 11}+\beta_{21} t_{i j}+\beta_{31} t_{i j}^{2}+\beta_{41} t_{i j}^{3}$ & -1050.86 \\
\hline 3 & $\beta_{11}+\gamma_{i 11}+\beta_{21} t_{i j}+\beta_{31} x_{i j 1}$ & $\mathbf{- 1 0 7 9 . 3 3}$ \\
& $\beta_{11}+\gamma_{i 11}+\beta_{21} t_{i j}+\beta_{31} x_{i j 2}$ & -1073.59 \\
& $\beta_{11}+\gamma_{i 11}+\beta_{21} t_{i j}+\beta_{31} x_{i j 3}$ & -1071.05 \\
& $\beta_{11}+\gamma_{i 11}+\beta_{21} t_{i j}+\beta_{31} x_{i j 4}$ & -1066.07 \\
& $\beta_{11}+\gamma_{i 11}+\beta_{21} t_{i j}+\beta_{31} x_{i j 1}+\beta_{41} x_{i j 2}$ & -1077.88 \\
& $\beta_{11}+\gamma_{i 11}+\beta_{21} t_{i j}+\beta_{31} x_{i j 1}+\beta_{41} x_{i j 3}$ & -1077.63 \\
& $\beta_{11}+\gamma_{i 11}+\beta_{21} t_{i j}+\beta_{31} x_{i j 1}+\beta_{41} x_{i j 4}$ & -1068.30 \\
\hline 4 & $\beta_{11}+\gamma_{i 11}+\beta_{21} t_{i j}+\beta_{31} x_{i j 1}+\beta_{41} t_{i j} x_{i j 1}$ & $\mathbf{- 1 0 7 7 . 5 0}$ \\
\hline 5 & $\beta_{11}+\gamma_{i 11}+\beta_{21} t_{i j}+\gamma_{i 21} t_{i j}+\beta_{31} x_{i j 1}$ & $\mathbf{- 1 0 7 9 . 4 7}$ \\
\hline Modelo Final & $\beta_{11}+\gamma_{i 11}+\beta_{21} t_{i j}+\gamma_{i 21} t_{i j}+\beta_{31} x_{i j 1}$ & $\mathbf{- 1 0 7 9 . 4 7}$ \\
\hline
\end{tabular}

Seja $y_{i j}$ a taxa de abandono escolar no ensino fundamental e médio no município $i$ observado no tempo $j$ com $i=1, \ldots, 61$ e $j=1, \ldots, 4$. De acordo com as metodologias de seleção de modelos o modelo beta selecionado foi o seguinte

$$
\begin{array}{r}
y_{i j} \mid \gamma_{i 1}, \gamma_{i 2} \stackrel{\text { ind }}{\sim} \operatorname{Be}\left(\mu_{i j}, \sigma_{i j}\right), \\
\gamma_{i 1} \stackrel{\text { i.i.d. }}{\sim} N\left(0, \Sigma_{1}\right), \\
\gamma_{i 2} \stackrel{\text { i.i.d }}{\sim} N\left(0, \lambda_{12}\right),
\end{array}
$$


Tabela 7.3: Modelos beta normal ajustados à estrutura de regressão de $\sigma$ e valores AIC.

\begin{tabular}{llc}
\hline Passos & Modelo & AIC \\
\hline 1 & $\beta_{12}+\gamma_{i 12}$ & $\mathbf{- 1 0 8 3 . 6 8}$ \\
\hline 2 & $\beta_{12}+\gamma_{i 12}+\beta_{22} t_{i j}$ & -1074.55 \\
& $\beta_{12}+\gamma_{i 12}+\beta_{22} t_{i j}+\beta_{32} t_{i j}^{2}$ & -1076.09 \\
& $\beta_{12}+\gamma_{i 12}+\beta_{22} t_{i j}+\beta_{32} t_{i j}^{2}+\beta_{42} t_{i j}^{3}$ & -1076.45 \\
\hline 3 & $\beta_{12}+\gamma_{i 12}+\beta_{22} x_{i j 1}$ & $\mathbf{- 1 0 8 3 . 8 7}$ \\
& $\beta_{12}+\gamma_{i 12}+\beta_{22} x_{i j 2}$ & -1081.49 \\
& $\beta_{12}+\gamma_{i 12}+\beta_{22} x_{i j 3}$ & -1071.75 \\
& $\beta_{12}+\gamma_{i 12}+\beta_{22} x_{i j 4}$ & -1067.42 \\
& $\beta_{12}+\gamma_{i 12}+\beta_{22} x_{i j 1}+\beta_{32} x_{i j 2}$ & -1073.87 \\
& $\beta_{12}+\gamma_{i 12}+\beta_{22} x_{i j 1}+\beta_{32} x_{i j 3}$ & -1077.45 \\
& $\beta_{12}+\gamma_{i 12}+\beta_{22} x_{i j 1}+\beta_{32} x_{i j 4}$ & -1067.41 \\
\hline Modelo final & $\beta_{12}+\gamma_{i 12}+\beta_{22} x_{i j 1}$ & $\mathbf{- 1 0 8 3 . 8 7}$ \\
\hline
\end{tabular}

em que $\gamma_{i 1}=\left(\gamma_{i 11}, \gamma_{i 21}\right)^{\top}$ e

$$
\begin{aligned}
& \operatorname{logito}\left(\mu_{i j}\right)=\beta_{11}+\gamma_{i 11}+\beta_{21} t_{i j}+\gamma_{i 21} t_{i j}+\beta_{31} x_{i j 1}, \\
& \operatorname{logito}\left(\sigma_{i j}\right)=\beta_{12}+\gamma_{i 12}+\beta_{22} x_{i j 1} .
\end{aligned}
$$

A Tabela 7.4 apresenta as estimativas, os erros padrões e os valores-p dos parâmetros do modelo (7.3) e (7.4). No modelo final usamos a estrutura de variância-covariância de componentes de variância $(\mathrm{CV})$ porque o método de otimização usado obteve convergência apenas com esta estrutura. Os valores das estimativas indicam que a taxa de abandono escolar no ensino fundamental e médio nas escolas públicas diminui com o aumento do tempo, isto é principalmente porque nos últimos anos a secretaria de educação de Antioquia desenvolveu estratégias para reduzir as taxas de abandono. Entretanto, a taxa de abandono escolar aumenta conforme a porcentagem da população em domicilios com necessidades básicas insatisfeitas aumenta, ou seja, como esperado a falta de recursos econômicos nas familias aumenta a taxa de abandono escolar. Notando que a variabilidade destas taxas também são explicadas pela porcentagem da população em domicilios com necessidades básicas insatisfeitas. nesta aplicação não faz sentido a interpretação da estimativa de $\beta_{11}$.

Tabela 7.4: Estimativas, erros padrões e valores-p do modelo beta normal ajustado aos dados de taxa de abandono escolar no ensino fundamental e médio.

\begin{tabular}{ccccc}
\hline & Parâmetro & Estimativa & ep & valor-p \\
\hline$\mu$ & $\beta_{11}$ & $-2,6688$ & 0,0974 & $2,0 \mathrm{e}-16$ \\
& $\beta_{21}$ & $-0,4470$ & 0,0222 & $2,0 \mathrm{e}-16$ \\
& $\beta_{31}$ & 1,1489 & 0,1810 & $1,1 \mathrm{e}-09$ \\
\hline$\sigma$ & $\beta_{12}$ & $-3,0081$ & 0,2150 & $2,0 \mathrm{e}-16$ \\
& $\beta_{22}$ & 1,6703 & 0,4834 & 0,0007 \\
\hline$\mu$ & $\log \left(\lambda_{11}\right)$ & $-1,4260$ & 0,1180 & \\
& $\log \left(\lambda_{21}\right)$ & $-1,4940$ & 0,0910 & \\
\hline$\sigma$ & $\log \left(\lambda_{12}\right)$ & $-0,7284$ & 0,1670 & \\
\hline
\end{tabular}


A Figura 7.7 apresenta os dados observados e a predição da taxa de abandono escolar no ensino fundamental e médio nos municípios de uma região escolhida arbitrariamente, a região L. Nota-se que as linhas sobrepostas seguem a tendência da taxa de abandono escolar nos municípios considerados, com exceção dos municípios 42 e 43 em que os dados observados e a predição estão afastados.

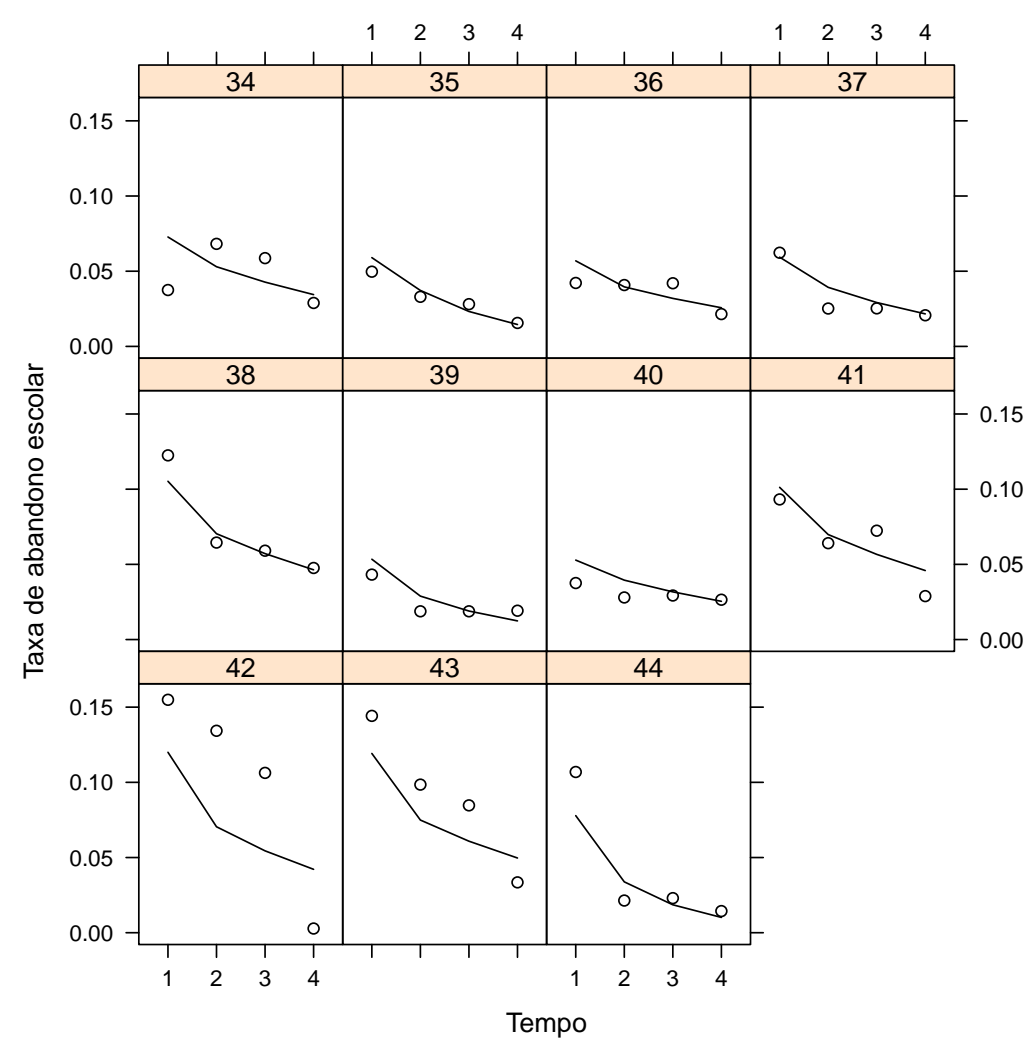

Figura 7.7: Dados observados e predição da taxa de abandono escolar no ensino fundamental $e$ médio nos municípios da região $L$.

\subsection{Conclusões}

O objetivo deste capítulo foi ilustrar a formulação, estimação, predição, seleção e análise de resíduos dos modelos propostos. Para isso apresentamos duas aplicações, na primeira formulamos um modelo de regressão beta com intercepto aleatório normal e usamos as ferramentas de diagnóstico propostas. Na segunda formulamos um modelo de regressão beta com intercepto e inclinação aleatória normal e utilizamos a metodologia de seleção de modelos proposta. Vale notar que, em modelos mais complexos em certos conjuntos de dados os métodos de otimização não garantem a convergência. De acordo com o que foi observado nos gráficos de dados e predições das variáveis respostas das duas aplicações podemos notar que os métodos de estimação dos parâmetros e de predição dos componentes aleatórios propostos são confiáveis e proporcionam bons resultados respeitando a especificidade dos dados. 


\section{Capítulo 8}

\section{Pacote BLMM}

O objetivo principal deste pacote é permitir o ajuste de modelos de regressão beta com efeitos aleatórios. Todas as funções foram implementadas na linguagem R. A função blmmML ajusta modelos de regressão beta com intercepto e inclinação aleatória. Esta função permite escolher a parametrização da distribuição beta, em termos da média e da precisão ou em termos da média e da dispersão (ver equações (2.7) e (2.8)). Também pode-se selecionar a função de ligação da média e do parâmetro de precisão ou de dispersão segundo seja a parametrização utilizada. A Tabela 3.1 apresenta exemplos de funções de ligação para o parâmetro $\mu$ que também podem ser usadas para o parâmetro de dispersão. Além disso, a função permite que seja escolhida a distribuição que seguem os efeitos aleatórios na média e no parâmetro de precisão ou de dispersão. Estão disponíveis seis distribuições de probabilidade para os interceptos aleatórios e cinco para o intercepto e a inclinação aleatória com estruturas de variância-covariância como as definidas na Tabela 5.1. A função usa o método de máxima verossimilhança para obter as estimativas do modelo utilizando os métodos de aproximação numérica da quadratura de Gauss-Hermite usual e adaptativa para resolver as integrais relacionadas ao processo inferencial. Para obter os pontos e pesos da quadratura de Gauss-Hermite implementamos também a função GHQ. A vantagem desta função sobre a função gauss. quad do pacote statmod é de permitir a eliminação de combinações de pontos que contribuem pouco à aproximação das integrais. A otimização do modelo é feita por meio da função nlminb ou optim do R. O pacote também contém as funções print.blmmML e summary.blmmML que permitem imprimir os resultados do ajuste do modelo e resumir objetos da classe blmmML.

A função re.prediction permite obter o melhor preditor dos efeitos aleatórios considerando diferentes funções de ligação, parametrizações da variável resposta, distribuições dos efeitos aleatórios e estruturas de variância-covariância. Outras duas funções importantes na análise de diagnóstico do modelo são as funções residuals.blmmML e halfnorm. A primeira, obtém os resíduos do modelo de regressão beta com intercepto aleatório e a segunda, baseada no pacote binomTools de Christensen e Hansen (2011), gera gráficos de 
probabilidade meio-normal com envelope simulado.

A seguir apresentamos a descrição, o uso, os argumentos, os detalhes, o valor e os exemplos de cada uma das funções implementadas no R.

blmmML Ajusta modelos lineares beta mistos

\section{Descrição}

blmmML ajusta modelos lineares beta com intercepto e inclinação aleatória pelo método de máxima verossimilhança.

Uso

blmmML (formula, sigma.formula, data, param = "BE_DISP",

link.mu = "logit", link.sigma = "logit",

re.dist.mu = "NORMAL", re.dist.sigma = "NORMAL",

varcov $=$ "CSH", method $=$ "GHQ", n.points $=10$,

optimizer = "nlminb", optim.method = "Nelder-Mead",

control $=$ list ()$, A=$ NULL, transf.par.disp $=$ FALSE)

\section{Argumentos}

formula uma fórmula linear de dois lados que descreve os termos fixos e aleatórios do modelo, a resposta é escrita no lado esquerdo do operador e os termos fixos e aleatórios, separados por +, são escritos no lado direito. O caractere de barra vertical | separa a expressão que contém a matriz de planejamento associada aos termos aleatórios e o fator de agrupamento.

sigma.formula uma fórmula linear para ajustar um modelo para o parâmetro $\sigma$. Os termos fixos e aleatórios, separados por +, são escritos no lado direito do operador $\sim$.

data um data. frame que contém as variáveis referidas em formula e sigma. formula.

param

tipo de parametrização da distribuição beta. Existem duas parametrizações, a primeira refere-se à distribuição beta que é caracterizada pela média e pela dispersão "BE_DISP" (opção padrão) e a segunda refere-se à distribuição beta que é caracterizada pela média e pela precisão "BE_PREC". 
link.mu

link.sigma

re.dist.mu

re.dist.sigma

varcov

method

n.points

optimizer

optim.method especificação da função de ligação do parâmetro $\mu$. As opções da função de ligação são: "logit"(opção padrão), "probit", "log-log", "clog-log" e "cauchit".

especificação da função de ligação do parâmetro $\sigma$. No caso de se usar a parametrização "BE_DISP" as opções da função de ligação são: "logit" (opção padrão), "probit", "log-log", "clog-log" e "cauchit" e no caso de se usar a parametrização "BE_PREC" as opções da função de ligação são: "log" (opção padrão), "sqrt" e "identity".

distribuição dos efeitos aleatórios de $\mu$. Se o modelo tem como componente aleatória o intercepto as opções para a distribuição dos efeitos aleatórios são: normal "NORMAL" (opção padrão), log-gama "LG", $t$-Student "T.STUDENT", Cauchy "CAUCHY", exponencial potência "EXP.POT" e Laplace "LAP LACE". Se o modelo tem como componente aleatória o intercepto e a inclinação as opções são: normal "NORMAL" (opção padrão), $t$ Student "T.STUDENT", Cauchy "CAUCHY", exponencial potência "EXP.POT" e Laplace "LAPLACE".

distribuição dos efeitos aleatórios de $\sigma$. As distribuições usadas para as componentes aleatórias em $\sigma$ são as mesmas que as usadas no argumento re.dist.mu.

estrutura de variância-covariância dos efeitos aleatórios de $\mu$ e $\sigma$. As opções de estrutura são: simple "S", componentes de variância "VC", autoregressiva de ordem 1 "AR-1" e de simetria composta heterogênea "CSH" (opção padrão).

método usado para obter as estimativas de máxima verossimilhança. Os métodos disponivéis são: quadratura de Gauss-Hermite "GHQ" (opção padrão) e quadratura de Gauss-Hermite adaptativa "AGHQ".

número inteiro positivo. Este argumento é utilizado quando o método "GHQ" é escolhido e define o número de pontos usados na quadratura de GaussHermite para aproximar o logaritmo da função de verossimilhança.

otimizador usado na maximização do logaritmo da função de verossimilhança. Os otimizadores são "nlminb" (opção padrão) e "optim".

método de otimização usado no "opt im". Os métodos são "Nelder-Mead" (opção padrão), "BFGS", "CG", "L-BFGS-B" e "SANN". Para maiores detalhes sobre os métodos ver a função optim. 
control

lista de parâmetros para controlar o processo do ajuste. Para maiores detalhes ver as funções nlminb e optim.

A mínimo valor admissível para a busca de parâmetros de dispersão dos efeitos aleatórios na escala usual.

transf.par.disp variável lógica. Se transf.par.disp=TRUE os parâmetros de dispersão dos efeitos aleatórios são estimados na escala log e o para o parâmetro de correlação utilizamos a transformación logística.

\section{Valor}

Retorna uma lista com os seguintes componentes

fit uma lista com os componentes da função nlminb ou optim.

Y um vetor com a variável resposta.

$\mathrm{X} 1$ uma matriz com variáveis explicativas associadas aos efeitos fixos de $\mu$.

$\mathrm{X} 2$ uma matriz com variáveis explicativas associadas aos efeitos fixos de $\sigma$.

Z1 uma matriz com variáveis explicativas associadas aos efeitos aleatórios de $\mu$.

$\mathrm{Z} 2$

uma matriz com variáveis explicativas associadas aos efeitos aleatórios de $\sigma$.

ndim.mu o número de efeitos aleatórios na estrutura de regressão de $\mu$.

ndim.sigma o número de efeitos aleatórios na estrutura de regressão de $\sigma$.

group um vetor com os grupos ou indivíduos do estudo.

quad.mu uma lista com os pontos e pesos da quadratura de Gauss-Hermite usados na avaliação das integrais em $\mu$.

quad.sigma uma lista com os pontos e pesos da quadratura de Gauss-Hermite usados na avaliação das integrais em $\sigma$.

link.mu a função de ligação usada na estrutura de regressão de $\mu$.

link.sigma a função de ligação usada na estrutura de regressão de $\sigma$.

param a parametrização da distribuição beta usada no ajuste do modelo.

trans.par.disp o valor da variável lógica transf.par.disp.

re.dist.mu a distribuição dos efeitos aleatórios de $\mu$. 
re.dist.sigma a distribuição dos efeitos aleatórios de $\sigma$.

varcov a estrutura de variância-covariância usada no ajuste do modelo.

optimizer o otimizador usado na maximização do logaritmo da função de verossimilhança.

Hessian a matriz de informação observada.

Converged o valor de uma variável lógica. Se Converged=TRUE o processo de estimação converge.

loglik o valor do logaritmo da função de verossimilhança.

aic o valor do critério de informação de Akaike (AIC).

re.pred.Mu as predições dos interceptos aleatórios de $\mu$.

re.pred.Sigma as predições dos interceptos aleatórios de $\sigma$.

\section{Exemplos}

\# Modelo com intercepto aleatório normal em mu e sigma set. seed (123)

rsb $\quad<-\operatorname{rsbeta}(\mathrm{N}=20, \mathrm{ni}=4, \operatorname{Betal}=\mathrm{c}(-1,1)$,

Beta2 $=c(-2,0.5), 11=1,12=0.5$,

link.mu = "logit", link.sigma = "logit",

param = "BE_DISP")

$\mathrm{Y} \quad<-$ rSb\$Y

$\mathrm{X} 1<-\operatorname{rsb} \$ \mathrm{X} 1$

$\mathrm{X} 2<-\operatorname{rsb} \$ \mathrm{X} 2$

Cluster $<-$ rsb\$Cluster

Datal <- data.frame (cbind(Y,X1, Cluster))

Fit $<-$ blmmML (formula $=\quad \sim$ time $+(1$ | Cluster $)$, sigma.formula $=\sim 1+$ Time $+(1$ | Cluster $)$, data = Data, param = "BE_DISP", link.mu = "logit", link.sigma = "logit", re.dist.mu = "NORMAL", re.dist.sigma = "NORMAL", method = "GHQ", n.points $=6$,

optimizer = "nlminb", control $=$ list (eval. $\max =1000$, iter.max $=1000$, trace $=0), \mathrm{A}=\mathrm{NULL}$, transf.par.disp $=$ FALSE 
Fit

\# Modelo com intercepto aleatório exponencial potência

\# em mu e sigma

set.seed (123)

rsb $\quad<-\operatorname{rsbeta}(\mathrm{N}=20, \mathrm{ni}=4, \operatorname{Betal}=\mathrm{c}(-1,1)$,

Beta2 $=\mathrm{c}(-2,0.5), \mathrm{v} 1=1, \mathrm{v} 2=1$,

link.mu = "logit", link.sigma = "logit",

param = "BE_DISP")

$\mathrm{Y} \quad<-r s b \$ Y$

$\mathrm{X} 1<-\operatorname{rsb} \$ \mathrm{X} 1$

$\mathrm{X} 2<-r s b \$ \mathrm{X} 2$

Cluster $<-$ rsb\$Cluster

Data $<-$ data.frame (cbind(Y,X1, Cluster))

Fit $\quad-$ blmmML (formula $=\quad \mathrm{Y} \sim 1+$ Time + $(1$ Cluster $)$,

sigma.formula $=\sim 1+$ Time $+(1$ | Cluster $)$,

data $=$ Data, param = "BE_DISP",

link.mu = "logit", link.sigma = "logit",

re.dist.mu = "EXP.POT",

re.dist.sigma $=$ "EXP.POT",

method = "GHQ",

n.points $=6$, optimizer $=$ "nlminb",

control $=$ list (eval.max $=1000$, iter.max $=1000$,

trace $=0), A=$ NULL,

transf.par.disp $=$ FALSE)

Fit

\# Modelo com intercepto e inclinação aleatória normal em mu e sigma set. seed (0098)

rsb $<-\operatorname{rsbeta}(\mathrm{N}=20, \mathrm{ni}=4$,

$$
\begin{aligned}
& \text { Betal }=c(-0.3,0.6), \operatorname{Beta} 2=c(-2,-1.5), \\
& 111=\operatorname{sqrt}(0.5), 112=\operatorname{sqrt}(0.3), \operatorname{rhol}=-0.5, \\
& 121=\operatorname{sqrt}(0.3), 122=\operatorname{sqrt}(0.5), \operatorname{rho}=-0.8, \\
& \text { link.mu = "logit", link.sigm = "logit", } \\
& \text { param = "BE_DISP") }
\end{aligned}
$$

$\mathrm{Y} \quad<-r s b \$ Y$

$\mathrm{X} 1<-r s b \$ \mathrm{X} 1$ 


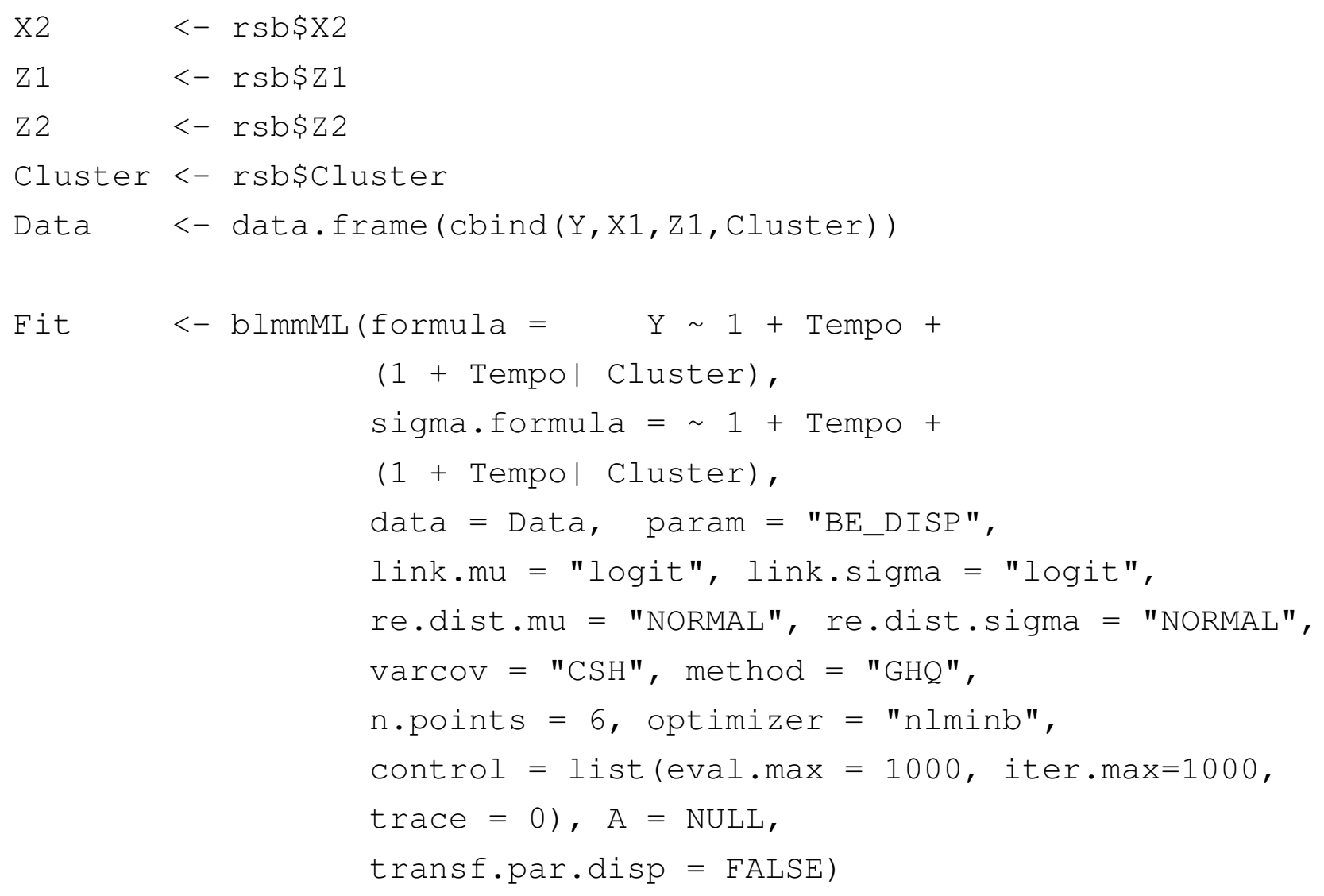

Fit

print.blmmML

Imprime um ajuste do modelo blmmML

\section{Descrição}

print.blmmML é o método do modelo beta com efeitos aleatórios específico para a função genérica print que imprime resultados de um objeto blmmML.

\section{Uso}

print.blmmML (object, digits $=\max (3$, getoption("digits") - 3), ...)

\section{Argumentos}

object

um objeto da classe blmmML.

digits

o número de dígitos significativos usados na impressão dos resultados.

\section{Valor}

Imprime os coficientes de um objeto blmmML.

\section{Exemplos}


\# Modelo com intercepto aleatório normal em mu e sigma set. seed (123)

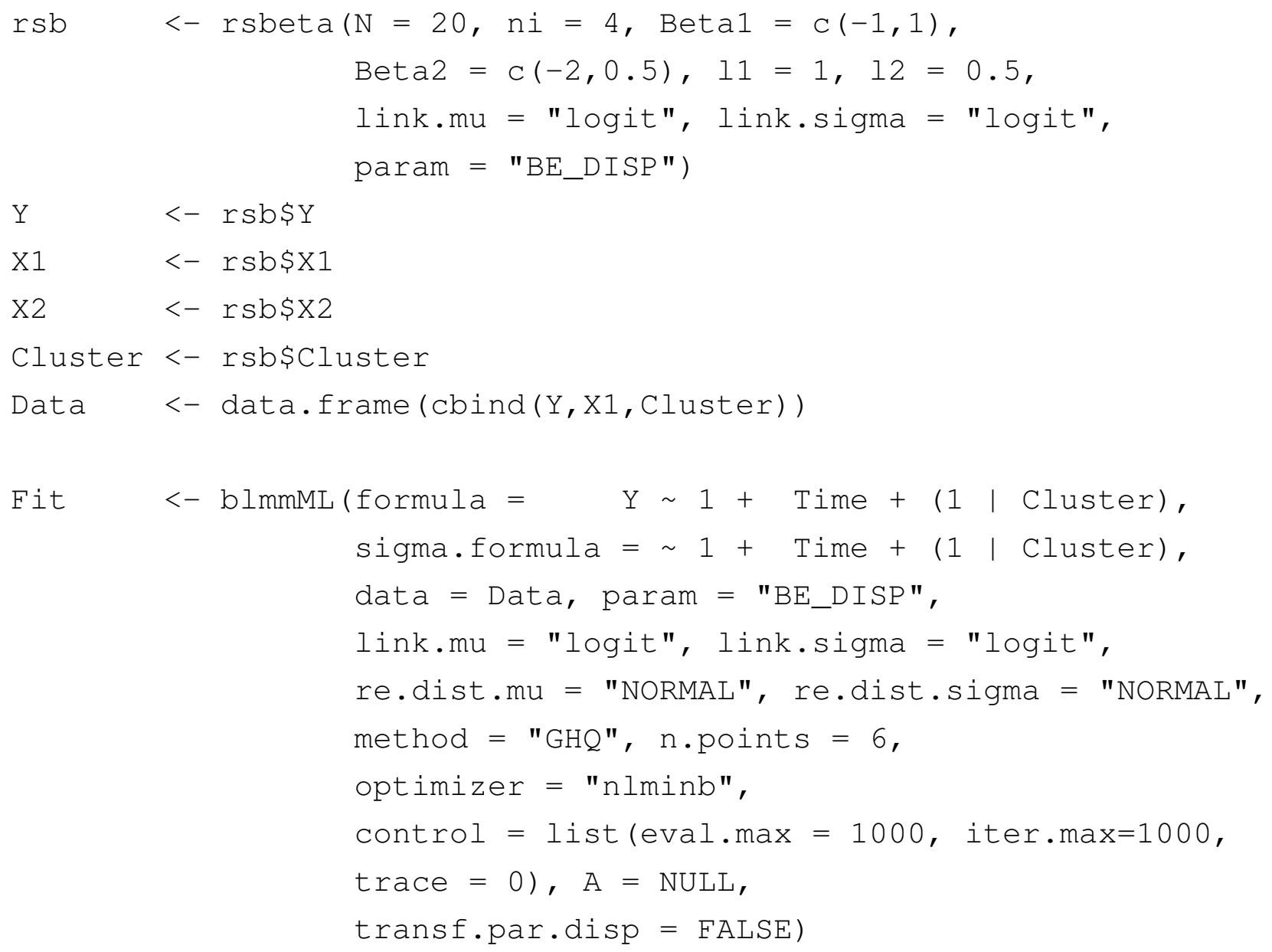

summary.blmmML

Resumo de um objeto blmmML

\section{Descrição}

summary.blmmML é o método do modelo beta com efeitos aleatórios específico para a função genérica summary que resume objetos da classe blmmML.

Uso

summary.blmmML (object, $\ldots$. )

\section{Argumentos}

object

um objeto da classe blmmML. 
... $\quad$ argumentos adicionais.

\section{Valor}

Imprime o resumo de um objeto blmmML.

\section{Exemplos}

\# Modelo com intercepto aleatório normal em mu e sigma set.seed (123)

rsb

$<-\operatorname{rsbeta}(\mathrm{N}=20, \mathrm{ni}=4, \operatorname{Betal}=\mathrm{c}(-1,1)$,

Beta2 $=c(-2,0.5), 11=1,12=0.5$,

link.mu = "logit", link.sigma = "logit",

param = "BE_DISP")

$\mathrm{Y} \quad<-r s b \$ Y$

$\mathrm{X} 1<-\operatorname{rsb} \$ \mathrm{X} 1$

$\mathrm{X} 2<-\operatorname{rsb} \$ \mathrm{X} 2$

Cluster $<-$ rsb\$Cluster

Data <- data.frame (cbind(Y,X1, Cluster))

Fit $<-$ blmmML (formula $=\quad \sim 1+$ Time $+(1 \mid$ Cluster),

sigma.formula $=\sim 1+$ Time $+(1$ | Cluster $)$,

data $=$ Data, param = "BE_DISP",

link.mu = "logit", link.sigma = "logit",

re.dist.mu = "NORMAL", re.dist.sigma = "NORMAL",

method $=$ "GHQ", $\mathrm{n}$.points $=6$,

optimizer = "nlminb",

control $=$ list (eval. $\max =1000$, iter. $\max =1000$,

trace $=0), A=$ NULL,

transf.par.disp = FALSE)

S $\quad<-$ summary.blmmML (Fit)

re.prediction

Predição dos interceptos aleatórios

\section{Descrição}

re.prediction é uma função interna da função blmmML que permite obter o melhor preditor dos efeitos aleatórios. 


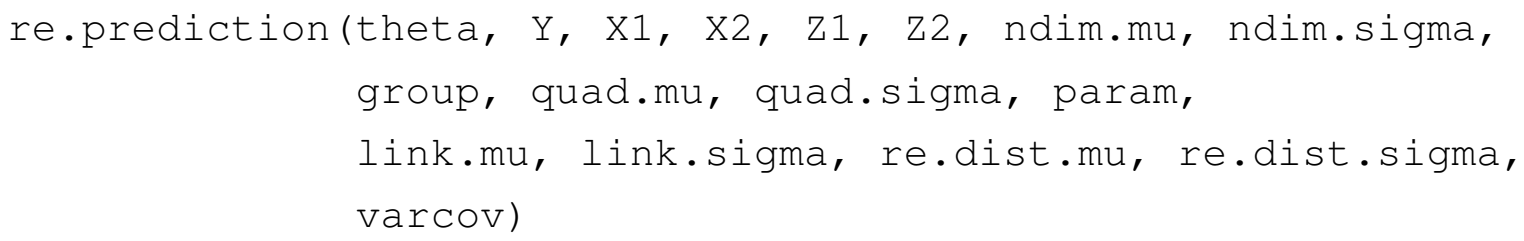

\section{Argumentos}

theta

$\mathrm{Y}$

$\mathrm{X} 1$

$\mathrm{X} 2$

Z1

$\mathrm{Z} 2$

ndim.mu

ndim.sigma

group

quad.mu

quad.sigma

param

link.mu

lik.sigma vetor com as estimativas de máxima verossimilhança.

vetor da variável resposta.

matriz com as variáveis explicativas associadas aos efeitos fixos de $\mu$.

matriz com as variáveis explicativas associadas aos efeitos fixos de $\sigma$.

matriz com as variáveis explicativas associadas aos efeitos aleatórios de $\mu$.

matriz com as variáveis explicativas associadas aos efeitos aleatórios de $\sigma$.

número de efeitos aleatórios considerados na estrutura de regressão de $\mu$.

número de efeitos aleatórios considerados na estrutura de regressão de $\sigma$.

vetor com os grupos o indivíduos do estudo.

uma lista com os pontos e os pesos da quadratura de Gauss-Hermite usados para resolver as integrais em $\mu$.

uma lista com os pontos e os pesos da quadratura de Gauss-Hermite usados para resolver as integrais em $\sigma$.

tipo de parametrização da distribuição beta. Existem duas parametrizações, a primeira refere-se à "BE_DISP" (opção padrão) e a segunda à "BE_PREC".

especificação da função de ligação do parâmetro $\mu$. As opções da função de ligação são: "logit"(opção padrão), "probit", "log-log" e "clog-log".

especificação da função de ligação do parâmetro $\sigma$. No caso de se usar a parametrização "BE_DISP" as opções da função de ligação são: "logit " (opção padrão), "probit", "log-log" e "clog-log" e no caso de se usar a parametrização "BE_PREC" a função de ligação disponível é a "log" (opção padrão). 
re.dist.mu

distribuição dos efeitos aleatórios de $\mu$. Se o modelo tem como componente aleatória o intercepto as opções para a distribuição dos efeitos aleatórios são: normal "NORMAL" (opção padrão), log-gama "LG", t-Student "T.STUDENT", Cauchy "CAUCHY", exponencial potência "EXP.POT" e Laplace "LAP LACE". Se o modelo tem como componente aleatória o intercepto e a inclinação as opções são: "NORMAL" (opção padrão), "T . STUDENT", "CAUCHY", "EXP.POT" e "LAPLACE".

re.dist.sigma distribuição dos efeitos aleatórios de $\sigma$. As distribuições usadas para as componentes aleatórias em $\sigma$ são as mesmas que as usadas no argumento re.dist.mu.

varcov estrutura de variância-covariância dos efeitos aleatórios de $\mu$ e $\sigma$. As opções de estrutura são: simple "S", componentes de variância "VC", autoregressiva de ordem 1 "AR-1" e de simetria composta heterogênea "CSH" (opção padrão).

\section{Valor}

Uma lista com as predições dos efeitos aleatórios em $\mu$ e $\sigma$.

\section{Exemplos}

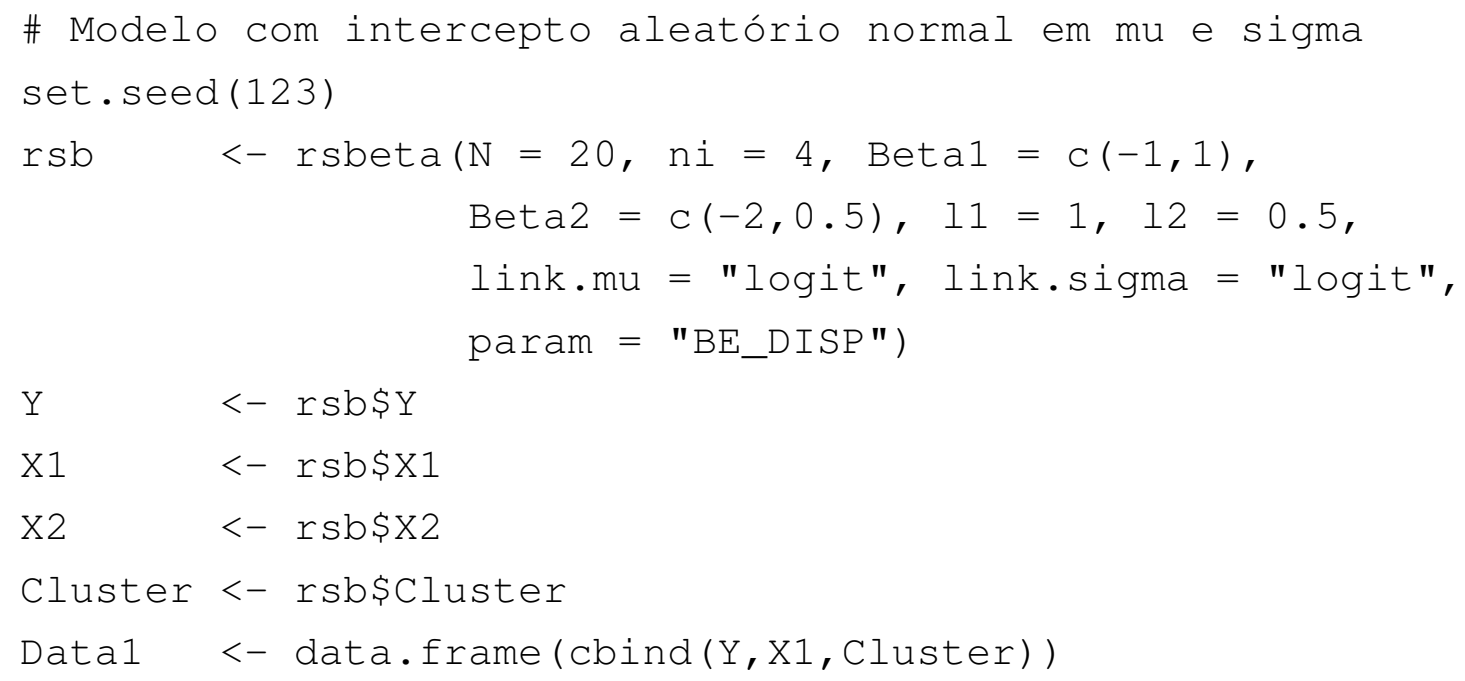




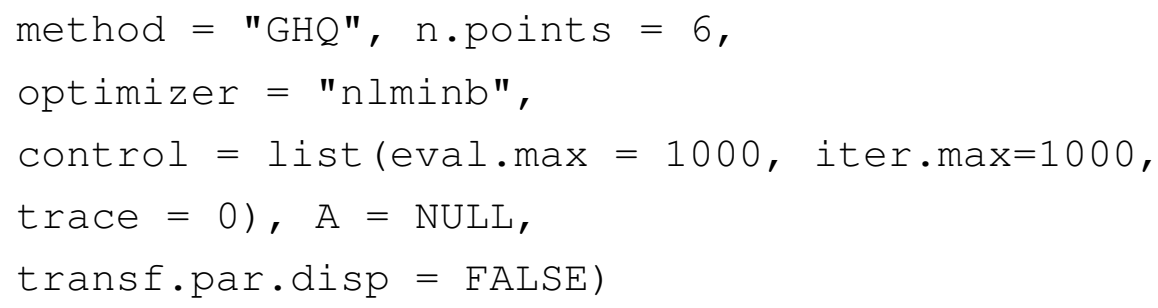

Fit\$re.pred.Mu

Fit\$re.pred.Sigma

GHQ

Quadratura de Gauss-Hermite

\section{Descrição}

GHQ calcula os pontos e os pesos da quadratura de Gauss-Hermite.

Uso

GHQ (n.pontos, ndim, pruning=TRUE)

\section{Argumentos}

n.pontos número de pontos usados na quadratura de Gauss-Hermite.

ndim número de efeitos aleatórios considerados na estrutura de regressão de $\mu$ e $\sigma$.

pruning variável lógica. Se pruning=TRUE são eliminadas as combinações de pontos que contribuem pouco à aproximação da integral.

\section{Detalhes}

Esta função está baseada na proposta de Hernández et al. (2013) que elimina as combinações de pontos da quadratura que contribuem pouco ao processo de aproximação da integral.

\section{Valor}

Uma lista com os pontos, pesos e o produto dos pesos da quadratura de Gauss-Hermite.

\section{Exemplos}

GHQ (n.pontos $=7$, ndim $=3$, pruning $=$ TRUE $)$ 
residuals.blmmML Resíduos do modelo de regressão beta com intercepto aleatório

\section{Descrição}

residuals.blmmML extrai os resíduos do modelo de regressão beta com intercepto aleatório.

\section{Uso}

residuals.blmmML (object, type = "quantil")

\section{Argumentos}

object

um objeto da classe blmmML.

type tipo de resíduo usado no gráfico. Os resíduos disponiveís são: "quantil", "condicional", "marginal", "random.mu" e "random.sigma".

\section{Detalhes}

Para detalhes dos resíduos ver Seção 6.2.

\section{Valor}

Um vetor de resíduos.

\section{Exemplos}

\# Modelo com intercepto aleatório normal em mu e sigma set. seed (123)

rsb $\quad<-\operatorname{rsbeta}(\mathrm{N}=20, \mathrm{ni}=4, \operatorname{Betal}=\mathrm{c}(-1,1)$,

$$
\text { Beta2 }=c(-2,0.5), 11=1,12=0.5 \text {, }
$$$$
\text { link.mu = "logit", link.sigma = "logit", }
$$
param = "BE_DISP")

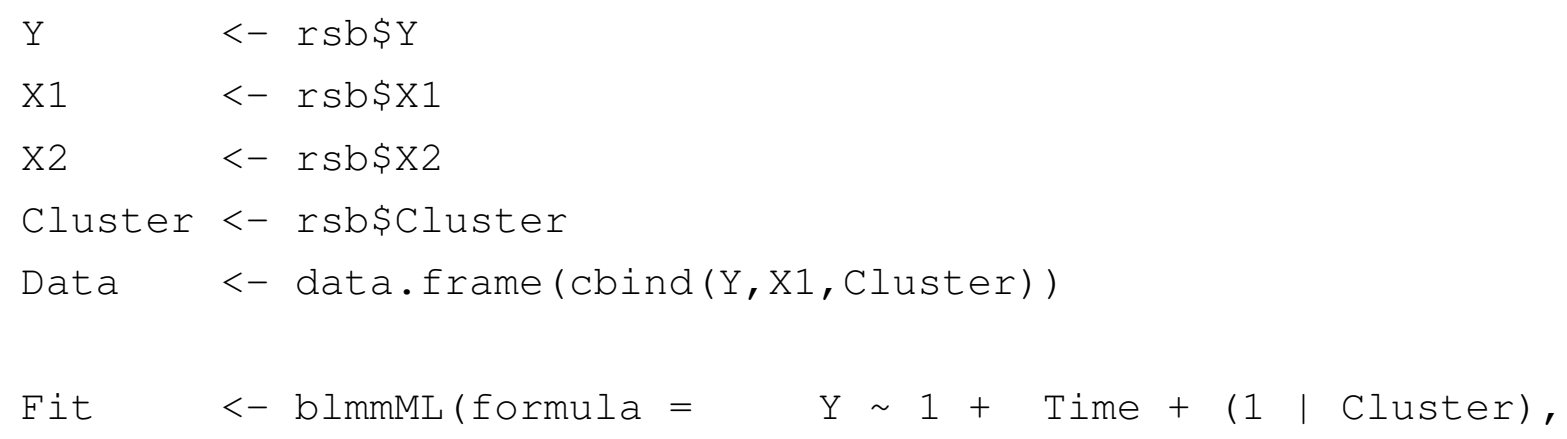




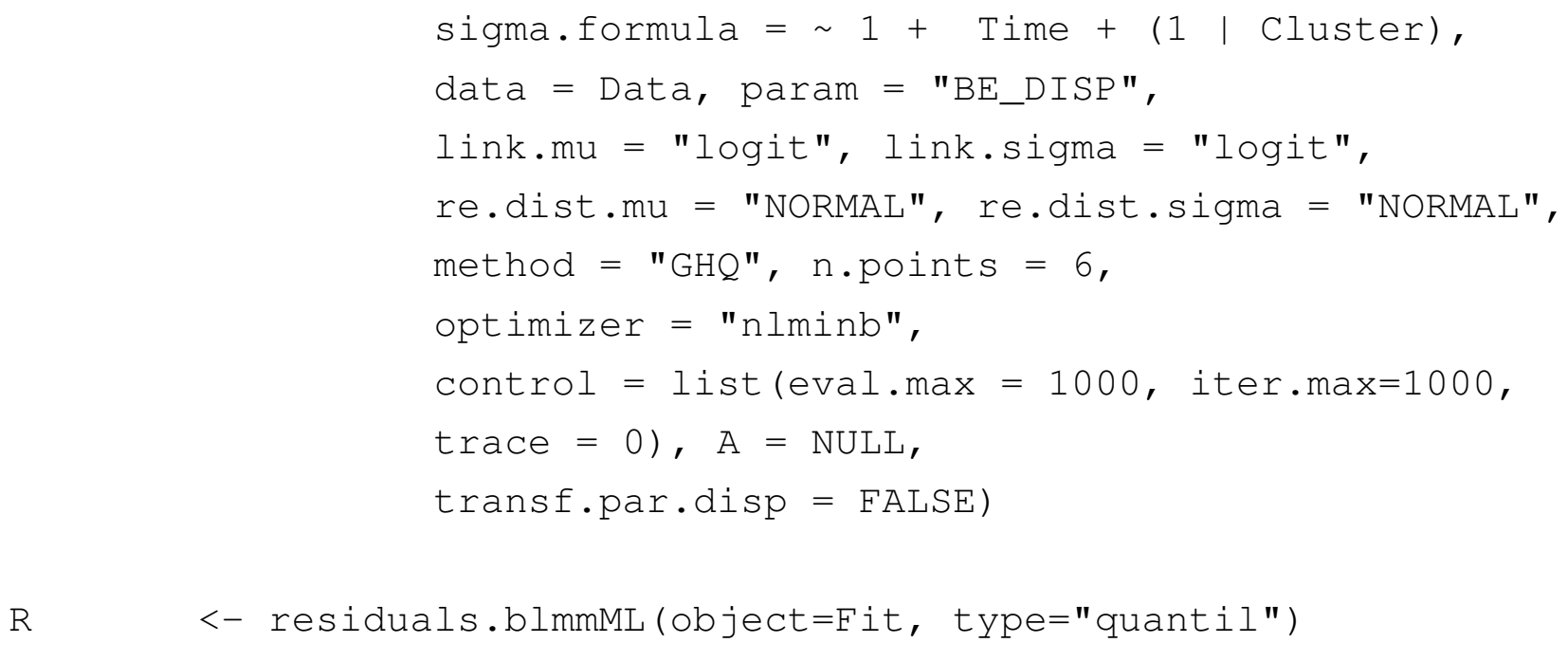

halfnorm

Gráficos de probabilidade meio-normal com envelope simulado

\section{Descrição}

halfnorm gera um gráfico de probabilidade meio-normal dos resíduos com envelopes simulados.

Uso

halfnorm(object, residual = "quantil", env = TRUE, nsim $=100$, plot $=$ TRUE, identify $=$ FALSE, $\mathrm{n}=2$ )

\section{Argumentos}

object um objeto da classe blmmML.

residual tipo de resíduo usado no gráfico. Os resíduos disponiveís são: "quantil", "condicional", "marginal", "random.mu" e "random.sigma".

env variável lógica. Se env=TRUE é gerado o envelope do resíduo escolhido.

nsim número inteiro positivo. Número de simulações usadas para gerar a banda de confiança. O valor padrão é 100 .

plot

variável lógica. Se plot=TRUE as bandas de confiança são traçadas no gráfico. Se plot=FALSE retorna uma lista com os valores das bandas de confiança.

identify

variável lógica. Se ident i fy=TRUE identifican-se pontos atípicos de forma interactiva no gráfico. 
$\mathrm{n}$

número inteiro positivo. Número de pontos que devem identificarse. Esse argumento é ignorado se identify $=$ FALSE.

\section{Detalhes}

Os valores absolutos dos resíduos são utilizados para construir o gráfico de probabilidade meio-normal.

\section{Valor}

Se plot=TRUE é obtido um gráfico. Se plot=FALSE a função retorna uma lista com os resíduos e seus valores esperados.

\section{Exemplos}

\# Modelo com intercepto aleatório normal em mu e sigma set. seed (123)

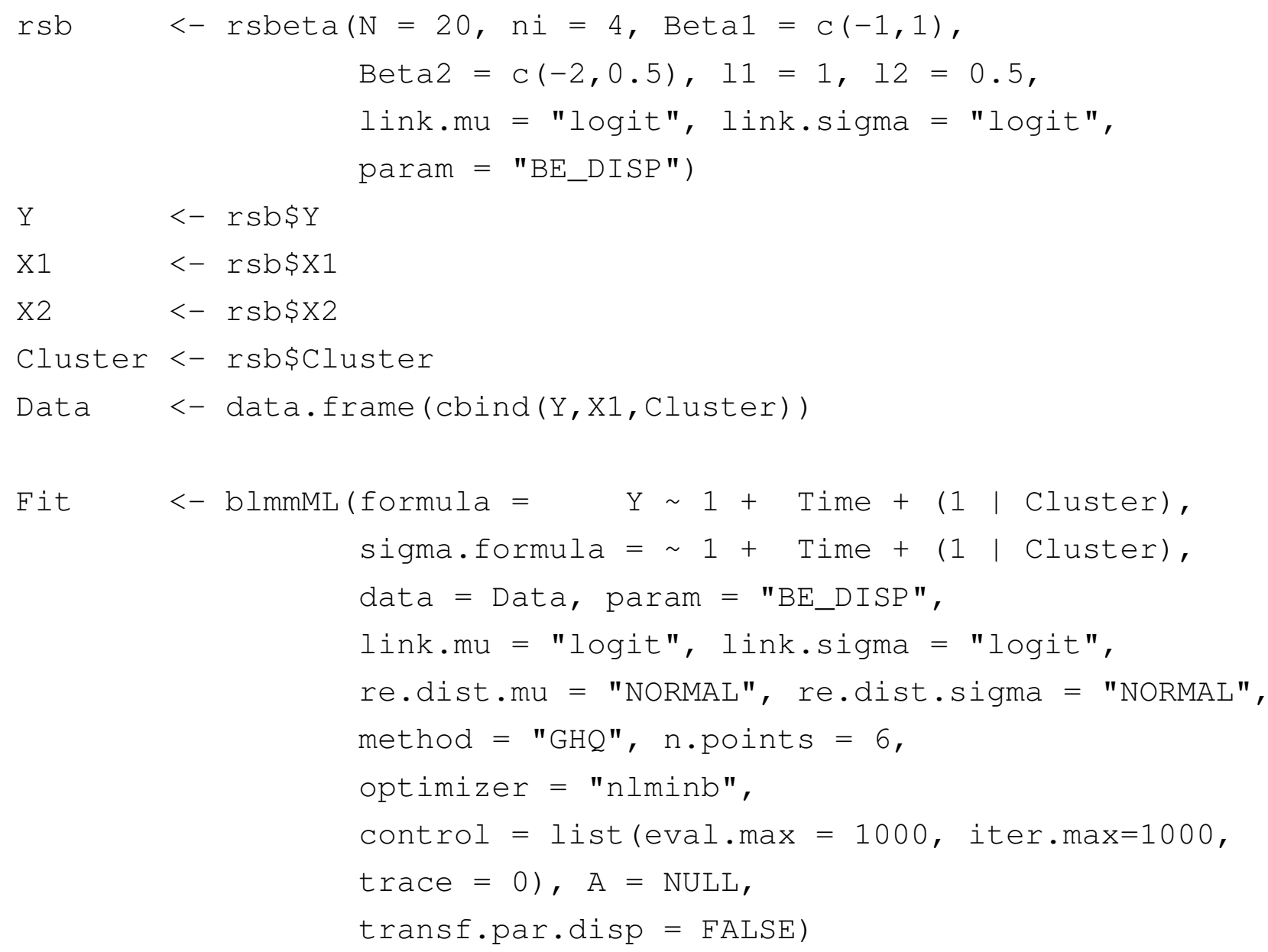




\section{Capítulo 9}

\section{Conclusões}

Neste trabalho propomos uma classe de modelos de regressão beta com interceptos aleatórios para dados que possuem uma distribuição beta e que são medidos ao longo do tempo. Os modelos estudados foram os modelos beta com interceptos aleatórios normal, log-gama, t-Student e exponencial potência. Estes modelos são muito versáteis devido a sua grande flexibilidade para capturar comportamentos normais, não normais e assimétricos que podem ocorrer no nível dos efeitos aleatórios. Para este tipo de modelos foram propostos os seguintes resíduos: quantil aleatorizado, marginal, condicional e aleatórios.

Foi proposta uma extensão para os modelos de regressão beta que consiste na inclusão de intercepto e inclinação aleatória na estrutura de regressão do modelo. Estes efeitos aleatórios seguem distribuições bivariadas pertencentes à classe das distribuições elípticas. Nestes modelos foram usadas as seguintes estruturas de variância-covariância: simples, de componentes de variância, auto-regressiva de ordem 1 e de simetria composta heterogênea. Com esta extensão a classe de modelos de regressão beta com efeitos aleatórios é enriquecida já que a correlação entre observações do mesmo indivíduo é acomodada de forma sistemática por meio das estruturas de variância-covariância.

A estimação conjunta dos parâmetros associados aos efeitos fixos e dos componentes de variância foi realizada por meio do método de máxima verossimilhança e foi utilizada a quadratura de Gauss Hermite para aproximar a função de verossimilhança. Esta metodologia de estimação permite obter os desvios padrões das componentes de variância que podem ser utilizados na construção de intervalos de confiança. A nossa proposta de estimação foi implementada no R por meio do pacote BLMM. Este pacote contém um conjunto de funções que facilitam aos usuários a estimação e análise de resíduos dos modelos propostos.

Estudos de simulação foram conduzidos para avaliar o processo de estimação dos parâmetros dos modelos de regressão beta tanto com interceptos quanto com interceptos e inclinações aleatórias. Para os modelos com interceptos aleatórios os resultados mostraram 
que os melhores resultados são encontrados quando o número de observações é grande e a variabilidade dos interceptos aleatórios é pequena. Para estes mesmos modelos encontramos as distribuições empíricas dos resíduos propostos e recomendamos o uso do resíduo quantil aleatorizado que segue uma distribuição aproximadamente normal. Para os modelos com intercepto e inclinação aleatória os resultados mostraram que o processo de estimação apresenta bons resultados quando o número de observações é grande e a estrutura de regressão do parâmetro de dispersão é simples.

Um procedimento para selecionar um modelo de regressão beta com efeitos aleatórios foi desenvolvido. Esta proposta consiste em selecionar de forma metódica as estruturas de regressão do modelo levando em conta a natureza longitudinal dos dados.

Assim, a metodologia apresentada é uma ferramenta completa e flexível para a análise de dados longitudinais que são medidos em escala contínua e são restritos a um intervalo fixado.

\subsection{Sugestões para Pesquisas Futuras}

Como possíveis trabalhos futuros propomos

- estender os estudos de simulação a fim de descrever o desempenho do processo de estimação, considerando a influência de covariáveis não temporais, dados perdidos, dados desbalanceados, número de pontos de quadratura e valores iniciais nas estimativas dos parâmetros dos modelos estudados,

- estender os estudos de simulação com o objetivo de estudar o comportamento das distribuições empíricas dos resíduos estudados e incorporar o resíduo padronizado 2 proposto por Espinheira et al. (2008),

- avaliar o tempo de processamento e as taxas de convergência do processo de estimação nos estudos de simulação propostos anteriormente,

- estudar o impacto da má especificação da distribuição dos efeitos aleatórios nas estimativas dos parâmetros dos modelos estudados,

- otimizar as funções propostas para os modelos com intercepto e inclinação aleatória,

- utilizar o teste escore e o teste de razão de verossimilhanças para estudar a inclusão dos efeitos aleatórios na modelagem dos parâmetro,

- estudar a eficiência do método de seleção de modelos proposto por meio de estudos de simulação, 
- estender os modelos propostos considerando funções não parametricas nos efeitos aleatórios e

- estender o trabalho para variáveis resposta com distribuição beta inflacionada. 
CONCLUSÕES 


\section{Apêndice A}

\section{Função escore e matriz de informação observada}

\section{A.1 Função escore}

Considerando a função densidade de probabilidade da distribuição beta com média $\mu$ e dispersão $\sigma$, denotada por $B e(\mu, \sigma)$, e definida como

$$
\begin{aligned}
f(y ; \mu, \sigma)= & \frac{\Gamma\left(\left(1-\sigma^{2}\right) / \sigma^{2}\right)}{\Gamma\left(\mu\left(\left(1-\sigma^{2}\right) / \sigma^{2}\right)\right) \Gamma\left((1-\mu)\left(\left(1-\sigma^{2}\right) / \sigma^{2}\right)\right)} y^{\mu\left(\left(1-\sigma^{2}\right) / \sigma^{2}\right)-1} \\
& \times(1-y)^{(1-\mu)\left(\left(1-\sigma^{2}\right) / \sigma^{2}\right)-1}
\end{aligned}
$$

com $0<y<1,0<\mu<1$ e $0<\sigma<1$, e considerando o modelo de regressão beta com intercepto aleatório normal dado em (3.1), o logaritmo da função de verossimilhança aproximada (3.16) pode ser expresso como

$$
\ell(\boldsymbol{\theta})=\sum_{i=1}^{N} \log B_{i}
$$

em que

$$
B_{i}=\left(\sum_{k_{1}=1}^{Q_{1}} \sum_{k_{2}=1}^{Q_{2}} A_{i} \frac{w_{k_{1}} w_{k_{2}}}{\pi}\right)
$$

$\mathrm{e}$

$$
\begin{aligned}
A_{i} & =\exp \left(\sum_{j=1}^{n_{i}} \log \Gamma\left(\frac{1-\sigma_{i j}^{2}}{\sigma_{i j}^{2}}\right)-\log \Gamma\left(\mu_{i j} \frac{(}{\left.1-\sigma_{i j}^{2}\right)} \sigma_{i j}^{2}\right)-\log \Gamma\left(\left(1-\mu_{i j}\right) \frac{\left(1-\sigma_{i j}^{2}\right)}{\sigma_{i j}^{2}}\right)\right. \\
& \left.+\left(\mu_{i j} \frac{1-\sigma_{i j}^{2}}{\sigma_{i j}^{2}}-1\right) \log y_{i j}+\left(\left(1-\mu_{i j}\right) \frac{1-\sigma_{i j}^{2}}{\sigma_{i j}^{2}}-1\right) \log \left(1-y_{i j}\right)\right)
\end{aligned}
$$

$\operatorname{com} g_{1}\left(\mu_{i j}\right)=\eta_{i j 1}=\boldsymbol{x}_{i j 1}^{\top} \boldsymbol{\beta}_{1}+\sqrt{2} \lambda_{1} z_{k_{1}}$ e $g_{2}\left(\sigma_{i j}\right)=\eta_{i j 2}=\boldsymbol{x}_{i j 2}^{\top} \boldsymbol{\beta}_{2}+\sqrt{2} \lambda_{2} z_{k_{2}}$. 
Diferenciando o logaritmo da função de verossimilhança (A.2) em relação a cada um dos parâmetros é obtida a função escore dada por $\boldsymbol{U}(\boldsymbol{\theta})=\left(\boldsymbol{U}_{\boldsymbol{\beta}_{1}}^{\top}(\boldsymbol{\theta}), \boldsymbol{U}_{\boldsymbol{\beta}_{2}}^{\top}(\boldsymbol{\theta}), U_{\lambda_{1}}(\boldsymbol{\theta}), U_{\lambda_{2}}(\boldsymbol{\theta})\right)^{\top}$, com

$$
\begin{aligned}
\boldsymbol{U}_{\boldsymbol{\beta}_{1}}(\boldsymbol{\theta}) & =\sum_{i=1}^{N} \frac{\boldsymbol{X}_{i 1}^{\top} \boldsymbol{D}_{i 1}}{B_{i}}, \\
\boldsymbol{U}_{\boldsymbol{\beta}_{2}}(\boldsymbol{\theta}) & =\sum_{i=1}^{N} \frac{\boldsymbol{X}_{i 2}^{\top} \boldsymbol{D}_{i 2}}{B_{i}}, \\
U_{\lambda_{1}}(\boldsymbol{\theta}) & =\sum_{i=1}^{N} \frac{D_{i 3}}{B_{i}}, \\
U_{\lambda_{2}}(\boldsymbol{\theta}) & =\sum_{i=1}^{N} \frac{D_{i 4}}{B_{i}},
\end{aligned}
$$

em que $\boldsymbol{X}_{i 1}$ e $\boldsymbol{X}_{i 2}$ são matrices de delineamento de ordens $\left(n_{i} \times p_{1}\right)$ e $\left(n_{i} \times p_{2}\right)$, respectivamente. Os vetores $n_{i}$-dimensionais $\boldsymbol{D}_{i 1}$ e $\boldsymbol{D}_{i 2}$ e as quantidades $D_{i 3}$ e $D_{i 4}$ são definidas a seguir.

O vetor $n_{i}$-dimensional $\boldsymbol{D}_{i 1}$ definido em $\boldsymbol{U}_{\boldsymbol{\beta}_{1}}(\boldsymbol{\theta})$ é dado por

$$
\boldsymbol{D}_{i 1}=\sum_{k_{1}=1}^{Q_{1}} \sum_{k_{2}=1}^{Q_{2}} A_{i} \boldsymbol{Q}_{i 1} \boldsymbol{P}_{i} \frac{w_{k_{1}} w_{k_{2}}}{\pi}
$$

$\operatorname{com} \boldsymbol{Q}_{i 1}=\operatorname{diag}\left\{d \mu_{i 1} / d \eta_{i 11}, \ldots, d \mu_{i n_{i}} / d \eta_{i n_{i} 1}\right\}$ e $\boldsymbol{P}_{i}=\left(p_{i 1}, \ldots, p_{i n_{i}}\right)^{\top}$, em que $p_{i j}=((1-$ $\left.\left.\sigma_{i j}^{2}\right) / \sigma_{i j}^{2}\right)\left(y_{i j}^{*}-\mu_{i j}^{*}\right), y_{i j}^{*}=\log \left\{y_{i j} /\left(1-y_{i j}\right)\right\}, \mu_{i j}^{*}=\psi\left(\mu_{i j}\left(\left(1-\sigma_{i j}^{2}\right) / \sigma_{i j}^{2}\right)\right)-\psi\left(\left(1-\mu_{i j}\right)((1-\right.$ $\left.\left.\left.\sigma_{i j}^{2}\right) / \sigma_{i j}^{2}\right)\right)$ e $\psi(\cdot)$ é a função digama definida como $\psi(z)=d \log \Gamma(z) / d z$, para $z>0$.

O vetor $n_{i}$-dimensional $\boldsymbol{D}_{i 2}$ definido em $\boldsymbol{U}_{\boldsymbol{\beta}_{2}}(\boldsymbol{\theta})$ é escrito como

$$
\boldsymbol{D}_{i 2}=\sum_{k_{1}=1}^{Q_{1}} \sum_{k_{2}=1}^{Q_{2}} A_{i} \boldsymbol{Q}_{i 2} \boldsymbol{R}_{i} \frac{w_{k_{1}} w_{k_{2}}}{\pi}
$$

$\operatorname{com} \boldsymbol{Q}_{i 2}=\operatorname{diag}\left\{d \sigma_{i 1} / d \eta_{i 12}, \ldots, d \sigma_{i n_{i}} / d \eta_{i n_{i} 2}\right\}$ e $\boldsymbol{R}_{i}=\left(r_{i 1}, \ldots, r_{i n_{i}}\right)^{\top}$, em que $r_{i j}=-2 a_{i j} / \sigma_{i j}^{3}$ e

$$
a_{i j}=\mu_{i j}\left(y_{i j}^{*}-\mu_{i j}^{*}\right)+\log \left(1-y_{i j}\right)+\psi\left(\frac{1-\sigma_{i j}^{2}}{\sigma_{i j}^{2}}\right)-\psi\left(\left(1-\mu_{i j}\right) \frac{1-\sigma_{i j}^{2}}{\sigma_{i j}^{2}}\right) .
$$

A quantidade $D_{i 3}$ definida em $U_{\lambda_{1}}(\boldsymbol{\theta})$ é dada por

$$
\boldsymbol{D}_{i 3}=\sum_{k_{1}=1}^{Q_{1}} \sum_{k_{2}=1}^{Q_{2}} A_{i} \boldsymbol{Q}_{i 1}^{*} \boldsymbol{P}_{i} \frac{\sqrt{2} z_{k_{1}} w_{k_{1}} w_{k_{2}}}{\pi}
$$

$\operatorname{com} \boldsymbol{Q}_{i 1}^{*}=\left(d \mu_{i 1} / d \eta_{i 11}, \ldots, d \mu_{i n_{i}} / d \eta_{i n_{i} 1}\right)$ 
A quantidade $D_{i 4}$ definida em $U_{\lambda_{2}}(\boldsymbol{\theta})$ é expressa por

$$
\boldsymbol{D}_{i 4}=\sum_{k_{1}=1}^{Q_{1}} \sum_{k_{2}=1}^{Q_{2}} A_{i} \boldsymbol{Q}_{i 2}^{*} \boldsymbol{R}_{i} \frac{\sqrt{2} z_{k_{2}} w_{k_{1}} w_{k_{2}}}{\pi}
$$

$\operatorname{com} \boldsymbol{Q}_{i 2}^{*}=\left(d \sigma_{i 1} / d \eta_{i 12}, \ldots, d \sigma_{i n_{i}} / d \eta_{i n_{i} 2}\right)$

\section{A.2 Matriz de informação observada}

A matriz de informação observada $\boldsymbol{J}_{\theta \theta}$ de ordem $(p \times p)$, com $p=p_{1}+p_{2}+2$, para o modelo beta com intercepto aleatório normal é dada por

$$
\left[\begin{array}{cccc}
\boldsymbol{J}_{\boldsymbol{\beta}_{1} \boldsymbol{\beta}_{1}} & \boldsymbol{J}_{\boldsymbol{\beta}_{1} \boldsymbol{\beta}_{2}} & \boldsymbol{J}_{\boldsymbol{\beta}_{1} \lambda_{1}} & \boldsymbol{J}_{\boldsymbol{\beta}_{1} \lambda_{2}} \\
\boldsymbol{J}_{\boldsymbol{\beta}_{2} \boldsymbol{\beta}_{1}} & \boldsymbol{J}_{\boldsymbol{\beta}_{2} \boldsymbol{\beta}_{2}} & \boldsymbol{J}_{\boldsymbol{\beta}_{2} \lambda_{1}} & \boldsymbol{J}_{\boldsymbol{\beta}_{2} \lambda_{2}} \\
\boldsymbol{J}_{\lambda_{1} \boldsymbol{\beta}_{1}} & \boldsymbol{J}_{\lambda_{1} \boldsymbol{\beta}_{2}} & J_{\lambda_{1} \lambda_{1}} & J_{\lambda_{1} \lambda_{2}} \\
\boldsymbol{J}_{\lambda_{2} \boldsymbol{\beta}_{1}} & \boldsymbol{J}_{\lambda_{2} \boldsymbol{\beta}_{2}} & J_{\lambda_{2} \lambda_{1}} & J_{\lambda_{2} \lambda_{2}}
\end{array}\right]
$$

em que $\boldsymbol{J}_{\theta \theta}$ é obtida como

$$
\begin{aligned}
\boldsymbol{J}_{\theta \theta} & =\sum_{i=1}^{N} \frac{\partial \boldsymbol{U}_{i}^{\top}}{\partial \boldsymbol{\theta}} \\
& =\sum_{i=1}^{N} \frac{\partial}{\partial \boldsymbol{\theta}}\left(\frac{1}{B_{i}} \frac{\partial B_{i}}{\partial \boldsymbol{\theta}^{\top}}\right) \\
& =\sum_{i=1}^{N}\left(\frac{1}{B_{i}} \frac{\partial^{2} B_{i}}{\partial \boldsymbol{\theta} \partial \boldsymbol{\theta}^{\top}}-\frac{1}{B_{i}^{2}} \frac{\partial B_{i}}{\partial \boldsymbol{\theta}} \frac{\partial B_{i}}{\partial \boldsymbol{\theta}^{\top}}\right) .
\end{aligned}
$$

Com base na equação (A.3) obtemos todas as derivadas apresentadas a seguir.

$$
\begin{aligned}
\boldsymbol{J}_{\boldsymbol{\beta}_{1} \boldsymbol{\beta}_{1}} & =-\sum_{i=1}^{N} \boldsymbol{X}_{i 1}^{\top}\left(\frac{\boldsymbol{D}_{i 1} \boldsymbol{D}_{i 1}^{\top}}{B_{i}^{2}}-\frac{\boldsymbol{D}_{i 5}}{B_{i}}\right) \boldsymbol{X}_{i 1} \\
\boldsymbol{J}_{\boldsymbol{\beta}_{1} \boldsymbol{\beta}_{2}} & =-\sum_{i=1}^{N} \boldsymbol{X}_{i 1}^{\top}\left(\frac{\boldsymbol{D}_{i 1} \boldsymbol{D}_{i 2}^{\top}}{B_{i}^{2}}-\frac{\boldsymbol{D}_{i 6}}{B_{i}}\right) \boldsymbol{X}_{i 2} \\
\boldsymbol{J}_{\boldsymbol{\beta}_{1} \lambda_{1}} & =-\sum_{i=1}^{N} \boldsymbol{X}_{i 1}^{\top}\left(\frac{\boldsymbol{D}_{i 1} \boldsymbol{D}_{i 3}}{B_{i}^{2}}-\frac{\boldsymbol{D}_{i 7}}{B_{i}}\right) \\
\boldsymbol{J}_{\boldsymbol{\beta}_{1} \lambda_{2}} & =-\sum_{i=1}^{N} \boldsymbol{X}_{i 1}^{\top}\left(\frac{\boldsymbol{D}_{i 1} \boldsymbol{D}_{i 4}}{B_{i}^{2}}-\frac{\boldsymbol{D}_{i 8}}{B_{i}}\right)
\end{aligned}
$$




$$
\begin{aligned}
\boldsymbol{J}_{\boldsymbol{\beta}_{2} \boldsymbol{\beta}_{2}} & =-\sum_{i=1}^{N} \boldsymbol{X}_{i 2}^{\top}\left(\frac{\boldsymbol{D}_{i 2} \boldsymbol{D}_{i 2}^{\top}}{B_{i}^{2}}-\frac{\boldsymbol{D}_{i 9}}{B_{i}}\right) \boldsymbol{X}_{i 2}, \\
\boldsymbol{J}_{\boldsymbol{\beta}_{2} \lambda_{1}} & =-\sum_{i=1}^{N} \boldsymbol{X}_{i 2}^{\top}\left(\frac{\boldsymbol{D}_{i 2} \boldsymbol{D}_{i 3}}{B_{i}^{2}}-\frac{\boldsymbol{D}_{i 10}}{B_{i}}\right) \\
\boldsymbol{J}_{\boldsymbol{\beta}_{2} \lambda_{2}} & =-\sum_{i=1}^{N} \boldsymbol{X}_{i 2}^{\top}\left(\frac{\boldsymbol{D}_{i 2} \boldsymbol{D}_{i 4}}{B_{i}^{2}}-\frac{\boldsymbol{D}_{i 11}}{B_{i}}\right) \\
\boldsymbol{J}_{\lambda_{1} \lambda_{1}} & =-\sum_{i=1}^{N}\left(\frac{\boldsymbol{D}_{i 3}^{2}}{B_{i}^{2}}-\frac{\boldsymbol{D}_{i 12}}{B_{i}}\right) \\
\boldsymbol{J}_{\lambda_{1} \lambda_{2}} & =-\sum_{i=1}^{N}\left(\frac{\boldsymbol{D}_{i 3} \boldsymbol{D}_{i 4}}{B_{i}^{2}}-\frac{\boldsymbol{D}_{i 13}}{B_{i}}\right) \\
\boldsymbol{J}_{\lambda_{2} \lambda_{2}} & =-\sum_{i=1}^{N}\left(\frac{\boldsymbol{D}_{i 4}^{2}}{B_{i}^{2}}-\frac{\boldsymbol{D}_{i 14}}{B_{i}}\right)
\end{aligned}
$$

em que as matrices $\boldsymbol{D}_{i 5}$ à $\boldsymbol{D}_{i 14}$ são definidas a seguir.

A matriz $\boldsymbol{D}_{i 5}$ de ordem $\left(n_{i} \times n_{i}\right)$ definida em $\boldsymbol{J}_{\boldsymbol{\beta}_{1} \boldsymbol{\beta}_{1}}$ é dada por

$$
\boldsymbol{D}_{i 5}=\sum_{k_{1}=1}^{Q_{1}} \sum_{k_{2}=1}^{Q_{2}} A_{i}\left(\boldsymbol{Q}_{i 1} \boldsymbol{P}_{i}\left(\boldsymbol{Q}_{i 1} \boldsymbol{P}_{i}\right)^{\top}+\boldsymbol{T}_{i}\right) \frac{w_{k_{1}} w_{k_{2}}}{\pi}
$$

$\operatorname{com} \boldsymbol{T}_{i}=\operatorname{diag}\left\{t_{i 1}, \ldots, t_{i n_{i}}\right\} \mathrm{e}$

$$
t_{i j}=\frac{1-\sigma_{i j}^{2}}{\sigma_{i j}^{2}}\left(\left(y_{i j}^{*}-\mu_{i j}^{*}\right) \frac{d^{2} \mu_{i j}}{d \eta_{i j 1}^{2}}-b_{i j} \frac{1-\sigma_{i j}^{2}}{\sigma_{i j}^{2}}\left(\frac{d \mu_{i j}}{d \eta_{i j 1}}\right)^{2}\right),
$$

em que $b_{i j}=\psi^{\prime}\left(\mu_{i j}\left(\left(1-\sigma_{i j}^{2}\right) / \sigma_{i j}^{2}\right)\right)+\psi^{\prime}\left(\left(1-\mu_{i j}\right)\left(\left(1-\sigma_{i j}^{2}\right) / \sigma_{i j}^{2}\right)\right)$ e $\psi^{\prime}(\cdot)$ é a função trigama.

A matriz $\boldsymbol{D}_{i 6}$ de ordem $\left(n_{i} \times n_{i}\right)$ definida em $\boldsymbol{J}_{\boldsymbol{\beta}_{1} \boldsymbol{\beta}_{2}}$ é expressada como

$$
\boldsymbol{D}_{i 6}=\sum_{k_{1}=1}^{Q_{1}} \sum_{k_{2}=1}^{Q_{2}} A_{i}\left(\boldsymbol{Q}_{i 1} \boldsymbol{P}_{i}\left(\boldsymbol{Q}_{i 2} \boldsymbol{R}_{i}\right)^{\top}+\boldsymbol{V}_{i} \boldsymbol{Q}_{i 1} \boldsymbol{Q}_{i 2}\right) \frac{w_{k_{1}} w_{k_{2}}}{\pi}
$$

$\operatorname{com} \boldsymbol{V}_{i}=\operatorname{diag}\left\{v_{i 1}, \ldots, v_{i n_{i}}\right\} \mathrm{e}$

$$
v_{i j}=-\frac{2}{\sigma_{i j}^{3}}\left(\left(y_{i j}^{*}-\mu_{i j}^{*}\right)-\frac{1-\sigma_{i j}^{2}}{\sigma_{i j}^{2}}\left(\mu_{i j} b_{i j}-\psi^{\prime}\left(\left(1-\mu_{i j}\right) \frac{1-\sigma_{i j}^{2}}{\sigma_{i j}^{2}}\right)\right)\right) .
$$

O vetor $n_{i}$-dimensional $\boldsymbol{D}_{i 7}$ definido em $\boldsymbol{J}_{\boldsymbol{\beta}_{1} \lambda_{1}}$ é dado por

$$
\boldsymbol{D}_{i 7}=\sum_{k_{1}=1}^{Q_{1}} \sum_{k_{2}=1}^{Q_{2}} A_{i}\left(\boldsymbol{Q}_{i 1} \boldsymbol{P}_{i} \boldsymbol{Q}_{i 1}^{*} \boldsymbol{P}_{i}+\boldsymbol{T}_{i}^{*}\right) \frac{\sqrt{2} z_{k_{1}} w_{k_{1}} w_{k_{2}}}{\pi}
$$


$\operatorname{com} \boldsymbol{T}_{i}^{*}=\left(t_{i 1}, \ldots, t_{i n_{i}}\right)^{\top}$

O vetor $n_{i}$-dimensional $\boldsymbol{D}_{i 8}$ definido em $\boldsymbol{J}_{\boldsymbol{\beta}_{1} \lambda_{2}}$ é dado por

$$
\boldsymbol{D}_{i 8}=\sum_{k_{1}=1}^{Q_{1}} \sum_{k_{2}=1}^{Q_{2}} A_{i}\left(\boldsymbol{Q}_{i 1} \boldsymbol{P}_{i} \boldsymbol{Q}_{i 2}^{*} \boldsymbol{R}_{i}+\boldsymbol{Q}_{i 1} \boldsymbol{Q}_{i 2} \boldsymbol{V}_{i}^{*}\right) \frac{\sqrt{2} z_{k_{2}} w_{k_{1}} w_{k_{2}}}{\pi}
$$

$\operatorname{com} \boldsymbol{V}_{i}^{*}=\left(v_{i 1}, \ldots, v_{i n_{i}}\right)^{\top}$

A matriz $\boldsymbol{D}_{i 9}$ de ordem $\left(n_{i} \times n_{i}\right)$ definida em $\boldsymbol{J}_{\boldsymbol{\beta}_{2} \boldsymbol{\beta}_{2}}$ é dada por

$$
\boldsymbol{D}_{i 9}=\sum_{k_{1}=1}^{Q_{1}} \sum_{k_{2}=1}^{Q_{2}} A_{i}\left(\boldsymbol{Q}_{i 2} \boldsymbol{R}_{i}\left(\boldsymbol{Q}_{i 2} \boldsymbol{R}_{i}\right)^{\top}+\boldsymbol{Z}_{i}\right) \frac{w_{k_{1}} w_{k_{2}}}{\pi}
$$

$\operatorname{com} \boldsymbol{Z}_{i}=\operatorname{diag}\left\{z_{i 1}, \ldots, z_{i n_{i}}\right\}$ e

$$
z_{i j}=\frac{-2}{\sigma_{i j}^{3}}\left(a_{i j} \frac{d^{2} \sigma_{i j}}{d \eta_{i j 2}^{2}}-\frac{1}{\sigma_{i j}}\left(3 a_{i j}-\frac{2}{\sigma_{i j}^{2}} c_{i j}\right)\left(\frac{d \sigma_{i j}}{d \eta_{i j 2}}\right)^{2}\right),
$$

em que

$$
c_{i j}=\mu_{i j}^{2} b_{i j}-2 \mu_{i j} \psi^{\prime}\left(\left(1-\mu_{i j}\right) \frac{1-\sigma_{i j}^{2}}{\sigma_{i j}^{2}}\right)-\psi^{\prime}\left(\frac{1-\sigma_{i j}^{2}}{\sigma_{i j}^{2}}\right)+\psi^{\prime}\left(\left(1-\mu_{i j}\right) \frac{1-\sigma_{i j}^{2}}{\sigma_{i j}^{2}}\right) .
$$

O vetor $n_{i}$-dimensional $\boldsymbol{D}_{i 10}$ definido em $\boldsymbol{J}_{\boldsymbol{\beta}_{2} \lambda_{1}}$ é dado por

$$
\boldsymbol{D}_{i 10}=\sum_{k_{1}=1}^{Q_{1}} \sum_{k_{2}=1}^{Q_{2}} A_{i}\left(\boldsymbol{Q}_{i 2} \boldsymbol{R}_{i} \boldsymbol{Q}_{i 1}^{*} \boldsymbol{P}_{i}+\boldsymbol{Q}_{i 1} \boldsymbol{Q}_{i 2} \boldsymbol{V}_{i}^{*}\right) \frac{\sqrt{2} z_{k_{1}} w_{k_{1}} w_{k_{2}}}{\pi}
$$

O vetor $n_{i}$-dimensional $\boldsymbol{D}_{i 11}$ definido em $\boldsymbol{J}_{\boldsymbol{\beta}_{2} \lambda_{2}}$ é expressado como

$$
\boldsymbol{D}_{i 11}=\sum_{k_{1}=1}^{Q_{1}} \sum_{k_{2}=1}^{Q_{2}} A_{i}\left(\boldsymbol{Q}_{i 2} \boldsymbol{R}_{i} \boldsymbol{Q}_{i 2}^{*} \boldsymbol{R}_{i}+\boldsymbol{Z}_{i}^{*}\right) \frac{\sqrt{2} z_{k_{1}} w_{k_{1}} w_{k_{2}}}{\pi}
$$

em que $\boldsymbol{Z}_{i}^{*}=\left(z_{i 1}, \ldots, z_{i n_{i}}\right)^{\top}$.

A quantidade $D_{i 12}$ definida em $J_{\lambda_{1} \lambda_{1}}$ é dada por

$$
\boldsymbol{D}_{i 12}=\sum_{k_{1}=1}^{Q_{1}} \sum_{k_{2}=1}^{Q_{2}} A_{i}\left(\boldsymbol{Q}_{i 1}^{*} \boldsymbol{P}_{i} \boldsymbol{Q}_{i 1}^{*} \boldsymbol{P}_{i}+\operatorname{tr}\left(\boldsymbol{T}_{i}^{*}\right)\right) \frac{2 z_{k_{1}}^{2} w_{k_{1}} w_{k_{2}}}{\pi}
$$


A quantidade $D_{i 13}$ definida em $J_{\lambda_{1} \lambda_{2}}$ é expressada como

$$
\boldsymbol{D}_{i 13}=\sum_{k_{1}=1}^{Q_{1}} \sum_{k_{2}=1}^{Q_{2}} A_{i}\left(\boldsymbol{Q}_{i 1}^{*} \boldsymbol{P}_{i} \boldsymbol{Q}_{i 2}^{*} \boldsymbol{R}_{i}+\operatorname{tr}\left(\boldsymbol{V}_{i}^{*}\right)\right) \frac{2 z_{k_{1}} z_{k_{2}} w_{k_{1}} w_{k_{2}}}{\pi}
$$

A quantidade $D_{i 14}$ definida em $J_{\lambda_{2} \lambda_{2}}$ é dada por

$$
\boldsymbol{D}_{i 14}=\sum_{k_{1}=1}^{Q_{1}} \sum_{k_{2}=1}^{Q_{2}} A_{i}\left(\boldsymbol{Q}_{i 2}^{*} \boldsymbol{R}_{i} \boldsymbol{Q}_{i 2}^{*} \boldsymbol{R}_{i}+\operatorname{tr}\left(\boldsymbol{Z}_{i}^{*}\right)\right) \frac{2 z_{k_{2}}^{2} w_{k_{1}} w_{k_{2}}}{\pi} .
$$




\section{Referências Bibliográficas}

Akaike(1974) H. Akaike. A new look at the statistical model identification. IEEE Transactions on Automatic Control, 19(6):716-723. Citado na pág. 60

Andrade(2007) A.C.G. de Andrade. Efeitos da especificação incorreta da função de ligação no modelo derregressão beta. Dissertação de Mestrado, Instituto de Matemática e Estatística, Universidade de São Paulo, Brasil. Citado na pág. 18, 21

Anholeto(2010) T. Anholeto. Resíduos de pearson melhorados em modelos de regressão beta. Dissertação de Mestrado, Instituto de Matemática e Estatística, Universidade de São Paulo, Brasil. Citado na pág. 59

Atkinson(1985) A.C. Atkinson. Plots, Transformations and Regression: An Introduction to Graphical Methods of Diagnostic Regression Analysis. Oxford University Press, New York. Citado na pág. 65

Bonat et al.(2012) W.H. Bonat, P.J.Jr. Ribeiro e W.M. Zeviani. Modelo beta com efeitos aleatórios. January 2012. Citado na pág. 19, 49, 54

Bozdogan(1987) H. Bozdogan. Model selection and Akaike's information criterion (AIC): The general theory and its analytical extensions. Psychometrica, 52(3):345-370. Citado na pág. 60

Branscum et al.(2007) A.D. Branscum, W.O. Johnson e M.C. Thurmond. Bayesian beta regression: applications to household expenditure data and genetic distance between footand-mouth disease viruses. Australian and New Zealand Journal of Statistics, 49(3):287301. Citado na pág. 18

Brostöm e Holmberg(2011) G. Brostöm e H. Holmberg. Generalized linear models with clustered data: Fixed and random effects models. Computational Statistics and Data Analysis, 55(12):3123-3134. Citado na pág. 28

Buckley(2003) J. Buckley. Estimation of models with beta-distributed dependent variables: A replication and extension of Paolino. Political Analysis, 11(2):204-205. Citado na pág. 18 
Camarinha(2002) J.A.F. Camarinha. Modelos Lineares Mistos: Estruturas de Matrizes de Variâncias e Covariâncias e Selećão de Modelos. Tese de Doutorado, Escola Superior de Agricultura Luis de Queiroz, Universidade de São Paulo, Brasil. Citado na pág. 50

Capobianco(2000) R. Capobianco. Robustness aspects of the generalizaed normal distribution. Quaderni di Statistica, 2:127-145. Citado na pág. 13

Christensen e Hansen(2011) R. H. B. Christensen e M. K. Hansen. binomTools: Performing diagnostics on binomial regression models, 2011. URL http://CRAN.R-project. org/package=binomTools. R package version 1.0-1. Citado na pág. 90

Cordeiro(2004) G.M. Cordeiro. On Pearson's residuals in generalized linear models. Statistics and Probability Letters, 66(3):213-219. Citado na pág. 59

Cox(1968) D.R. Cox. Notes on some aspects of regression analysis. Journal of the Royal Statistical Society. Series A, 131(3):265-279. Citado na pág. 59

Cribari-Neto e Souza(2012) F. Cribari-Neto e T.C. Souza. Testing inference in variable dispersion beta regressions. Journal of Statistical Computation and Simulation, 82(12): 1827-1843. Citado na pág. 12, 18

Cribari-Neto e Zeileis(2010) F. Cribari-Neto e A. Zeileis. Beta regression in R. Journal of Statistical Software, 34(2):1-24. Citado na pág. 18, 27

Dunn e Smyth(1996) P.K. Dunn e G.K. Smyth. Randomized quantile residuals. Journal of Computational and Graphical Statistics, 5(3):236-244. Citado na pág. 59

Espinheira et al.(2008) P.L. Espinheira, S.L.P. Ferrari e F. Cribari-Neto. On beta regression residuals. Journal of Applied Statistics, 35(4):407-419. Citado na pág. 59, 106

Evans e Swartz(2000) M. Evans e T.B. Swartz. Approximating Integrals via Monte Carlo and Deterministic Methods. Oxford University Press Inc., New York. Citado na pág. 11

Fabio et al.(2012) L.C. Fabio, G.A. Paula e M. de Castro. A Poisson mixed model with nonnormal random effect distribution. Computational Statistics and Data Analysis, 56 (6):1499-1510. Citado na pág. 35

Fahrmeir e Tutz(2001) L. Fahrmeir e G. Tutz. Multivariate Statistical Modelling Based on Generalized Linear Models. Springer, New York, segunda edição. Citado na pág. 10, 11

Fang et al.(1990) K.T. Fang, S. Kotz e K.W. Ng. Symmetric Multivariate and Related Distributions. Chapman \& Hall, New York. Citado na pág. 13, 15

Ferrari e Cribari-Neto(2004) S.L.P. Ferrari e F. Cribari-Neto. Beta regression for modeling rates and proportions. Journal of Applied Statistics, 31(7):799-815. Citado na pág. 2, $12,17,18,19,59$ 
Ferrari e Pinheiro(2011) S.L.P. Ferrari e E.C. Pinheiro. Improved likelihood inference in beta regression. Journal of Statistical Computation and Simulation, 81(4):431-443. Citado na pág. 18

Figueroa-Zúñiga et al.(2012) Z.J.I. Figueroa-Zúñiga, R.B. Arellano-Valle e S.L.P. Ferrari. Mixed beta regression: a bayesian perspective. Novembro 2012. Citado na pág. 2, 19, 49

Fitzmaurice et al.(2009) G. Fitzmaurice, M. Davidian, G. Verbeke e G. Molenberghs. Longitudinal Data Analysis. Chapman \& Hall/CRC, Florida. Citado na pág. 1, 28

Fitzmaurice et al.(2004) G.M. Fitzmaurice, N.M. Laird e J.H. Ware. Applied Longitudinal Analysis. Wiley-Interscience, New Jersey. Citado na pág. 8

Fu e Wang(2012) L. Fu e Y.-G. Wang. Quantile regression for longitudinal data with a working correlation model. Computational Statistics and Data Analysis, 56(8):2526-2538. Citado na pág. 30

Grün et al.(2012) B. Grün, I. Kosmidis e A. Zeilis. Extended beta regression in R: shaken, stirred, mixed and partitioned. Journal of Statistical Software, 48(11):1-25. Citado na pág. 18

Guoyou e Zhongyi(2008) Q. Guoyou e Z. Zhongyi. Robust estimation in partial linear mixed model for longitudinal data. Acta Mathematica Scientia, 28(2):333-347. Citado na pág. 30

Hannan e Quin(1979) E.J. Hannan e G.G. Quin. The determination of the order of an autoregression. Journal of the Royal Statistical Society, Series B, 41(2):190-195. Citado na pág. 60

Hedeker e Gibbons(1994) D. Hedeker e R.D. Gibbons. A random-effects ordinal regression model for multilevel analysis. Biometrics, 50(4):933-944. Citado na pág. 27

Hernández et al.(2013) F. Hernández, O. Usuga e V. Giampaoli. Improving the adaptive gaussian quadrature. January 2013. Citado na pág. 11, 27, 100

Hilden-Minton(1995) Hilden-Minton. Multilevel Diagnostics for Mixed and Hierarchical Linear Models. Tese de Doutorado, University of California, USA. Citado na pág. 59

Hurvich e Tsai(1989) C. M. Hurvich e C.L. Tsai. Regression and time series model selection in small samples. Biometrika, 76(2):297-307. Citado na pág. 60

Kieschnick e McCullough(2003) R. Kieschnick e B.D. McCullough. Regression analysis of variates observed on (0,1): percentages, proportions, and fractions. Statistical Modelling, 3(3):193-213. Citado na pág. 2, 17 
Kutner et al.(2005) M.H. Kutner, C.J. Nachtsheim, J. Neter e W. Li. Applied Linear Statistical Models. McGraw-Hill, New York, quinta edição. Citado na pág. 65

Laird e Ware(1982) N.M. Laird e J.H. Ware. Random-effects models for longitudinal data. Biometrics, 38(4):963-974. Citado na pág. 1, 7, 9

Lin e Lee(2008) T.I. Lin e J.C. Lee. Estimation and prediction in linear mixed models with skew-normal random effects for longitudinal data. Statistics in Medicine, 27(9):1490-1507. Citado na pág. 35

Lindstrom e Bates(1988) M.J. Lindstrom e D.M. Bates. Newton-Raphson and EM algorithms for linear mixed-effects models for repeated-measures data. Journal of the American Statistical Association, 83(404):1014-1022. Citado na pág. 9

Littell et al.(2000) R. Littell, J. Pendergast e R. Natrajan. Modelling covariance structure in the analysis of repeated measures data. Statistics in Medicine, 19(13):1793-1819. Citado na pág. 8,50

Liu e Yu(2008) L. Liu e Z. Yu. A likelihood reformulation method in non-normal random effects models. Statistics in Medicine, 27(16):3105-3124. Citado na pág. 36, 39, 40, 53

Liu e Pierce(1994) Q. Liu e D.A. Pierce. A note on Gauss-Hermite quadrature. Biometrika, 81(3):624-629. Citado na pág. 11

Manghi(2011) R.F. Manghi. Modelos elípticos multiníveis. Dissertação de Mestrado, Instituto de Matemática e Estatística, Universidade de São Paulo, Brasil. Citado na pág. 49

McCullagh e Nelder(1983) P. McCullagh e J.A. Nelder. Generalized Linear Models. Chapman and Hall, London. Citado na pág. 21

McCulloch e Neuhaus(2011) C.E. McCulloch e J.M. Neuhaus. Misspecifying the shape of a random effects distribution: why getting it wrong Mmy not matter. Statistical Science, 26(3):388-402. Citado na pág. 35

Meyers et al.(1992) S.M. Meyers, J.S. Ambler, M. Tan, J.C. Werner e S.S. Huang. Variation of perfluorproane disappearance after vitrectomy. Retina, 12:359-363. Citado na pág. 77

Meza et al.(2012) C. Meza, F. Osorio e R. De la Cruz. Estimation in nonlinear mixedeffects models using heavy-tailed distributions. Statistics and Computing, 22(1):121-139. Citado na pág. 49

Molenberghs e Verbeke(2005) G. Molenberghs e G. Verbeke. Models for Discrete Longitudinal Data. Springer, New York. Citado na pág. 9, 28

Nobre e Singer(2007) J.S. Nobre e J.M. Singer. Residual analysis for linear mixed models. Biometrical Journal, 49(6):863-875. Citado na pág. 59 
Ospina e Ferrari(2012) R. Ospina e S.L.P. Ferrari. A general class of zero-or-one inflated beta regression models. Computational Statistics and Data Analysis, 56(6):1609-1623. Citado na pág. 18

Paolino(2001) P. Paolino. Maximum likelihood estimation of models with beta-distributed dependent variables. Political Analysis, 9(4):325-346. Citado na pág. 2, 17, 18

Park e Wu(2006) J.-G. Park e H. Wu. Backfitting and local likelihood methods for nonparametric mixed-effects models with longitudinal data. Journal of Statistical Planning and Inference, 136(11):3760-3782. Citado na pág. 30

Pinheiro e Bates(2000) J.C. Pinheiro e D.M. Bates. Mixed-Effects Models in $S$ and S-PLUS. Springer-Verlag, New York. Citado na pág. 8, 59

Pinheiro e Bates(1995) J.C. Pinheiro e D.M. Bates. Approximations to the log-likelihood function in the nonlinear mixed-effects model. Journal of Computational and Graphical Statistics, 4(1):12-35. Citado na pág. 11

Pinheiro et al.(2001) J.C. Pinheiro, C. Liu e Y.N. Wu. Efficient algorithms for robust estimation in linear mixed-effects models using the multivariate $t$ distribution. Journal of Computational and Graphical Statistics, 10(2):249-276. Citado na pág. 35

Qiu et al.(2008) Z. Qiu, P.X.-K. Song e M. Tan. Simplex mixed-effects models for longitudinal proportional data. Scandinavian Journal of Statistics, 35(4):577-596. Citado na pág. $77,79,80$

R Development Core Team(2012) R Development Core Team. R: A Language and Environment for Statistical Computing. R Foundation for Statistical Computing, Vienna, Austria, 2012. URL http://www.R-project.org. ISBN 3-900051-07-0. Citado na pág. 18

Rigby e Stasinopoulos(2005) R.A. Rigby e D.M. Stasinopoulos. Generalized additive models for location, scale and shape. Applied Statistical, 54(3):507-554. Citado na pág. 2, 19, 27

Rocha e Simas(2011) A.V. Rocha e A.B. Simas. Influence diagnostics in a general class of beta regression models. TEST, 20(1):95-119. Citado na pág. 59

Rocha(2004) F.M.M. Rocha. Seleção de estruturas de covariância em dados com medidas repetidas. Dissertação de Mestrado, Instituto de Matemática e Estatística, Universidade de São Paulo, Brasil. Citado na pág. 8

Russo(2010) N.C.M. Russo. Modelos Não Lineares Elípticos para Dados Correlacionados. Tese de Doutorado, Instituto de Matemática e Estatística, Universidade de São Paulo, Brasil. Citado na pág. 49 
Ryoo(2010) J.H. Ryoo. Model Selection with the Linear Mixed Effects Model for Longitudinal Data. Tese de Doutorado, University of Minnesota, USA. Citado na pág. 60

Schwarz(1978) G. Schwarz. Estimating the dimensional of a model. Annals of Statistics, 6(2):461-464. Citado na pág. 60

Searle et al.(2006) S.R. Searle, G. Casella e C.E. McCulloch. Variance Components. John Wiley \& Sons, New Jersey. Citado na pág. 50

Simas e Cordeiro(2009) A.B. Simas e G.M. Cordeiro. Adjusted Pearson residuals in exponential family nonlinear models. Journal of Statistical Computation and Simulation, 79(4):411-425. Citado na pág. 59

Simas et al.(2010) A.B. Simas, W. Barreto-Souza e A.V. Rocha. Improved estimators for a general class of beta regression models. Computational Statistics and Data Analysis, 54 (2):348-366. Citado na pág. 2, 17, 18

Smithson e Verkuilen(2006) M. Smithson e J. Verkuilen. A better lemon squeezer? maximum-likelihood regression with beta-distributed dependent variables. Psychological Methods, 11(1):54-71. Citado na pág. 2, 17

Solaro e Ferrari(2007) N. Solaro e P.A. Ferrari. Robustness of parameter estimation procedures in multilevel models when random effects are mep distributed. Statistical Methods and Applications, 16(1):51-67. Citado na pág. 49

Song(2007) P.X.-K. Song. Correlated Data Analysis: Modeling, Analytics, and Applications. Springer, New York. Citado na pág. 77, 79

Song e Tan(2000) P.X.-K. Song e M. Tan. Marginal models for longitudinal continuous proportional data . Biometrics, 56(2):496-502. Citado na pág. 77, 79

Song et al.(2004) P.X.-K. Song, Z. Qiu e M. Tan. Modelling heterogeneous dispersion in marginal models for longitudinal proportional data. Biometrical Journal, 46(5):540-553. Citado na pág. 77

Stasinopoulos et al.(2012) M. Stasinopoulos, B. Rigby e C. Akantziliotou. Instructions on How to Use the gamlss Package in R, segunda edição, 2012. URL http://www.R-project. org/. Citado na pág. 60

Venezuela(2008) M.K. Venezuela. Equação de Estimação Generalizada e Influência Local para Modelos de Regressão Beta com Medidas Repetidas. Tese de Doutorado, Instituto de Matemática e Estatística, Universidade de São Paulo, Brasil. Citado na pág. 2, 17, 77, 79

Verbeke e Lesaffre(1996) G. Verbeke e E. Lesaffre. A linear mixed-effects model with heterogeneity in the random-effects population. Journal of the American Statistical Association, 91(433):217-221. Citado na pág. 59 
Verbeke e Lesaffre(1997) G. Verbeke e E. Lesaffre. The effect of misspecifying the random effects distribution in linear mixed models for longitudinal data. Computational Statistics and Data Analysis, 23(4):541-556. Citado na pág. 35

Verbeke e Molenberghs(2009) G. Verbeke e G. Molenberghs. Linear Mixed Models for Longitudinal Data. Springer-Verlang, New York, segunda edição. Citado na pág. 5, 9, 28

Verbeke et al.(2001) G. Verbeke, B. Spiessens e E. Lessafre. Conditional linear mixed models. The American Statistician, 55(1):25-34. Citado na pág. 9

Verkuilen e Smithson(2012) J. Verkuilen e M. Smithson. Mixed and mixture regression models for continuos bounded responses using the beta distribution. Journal of Educational and Behavioral Statistics, 37(1):82-113. Citado na pág. 19, 49

Wei e Zhang(2009) H. Wei e W. Zhang. Maximum likelihood estimation for simplex distribution nonlinear mixed models via an EM algorithm. International Journal of Biomathematics, 2(1):9-17. Citado na pág. 77,79

Weiss(2005) R.E. Weiss. Modeling Longitudinal Data. Springer, New York. Citado na pág. 8

Wissel(2009) J. Wissel. A New Biased Estimator for Multivariate Regression Models with Highly Collinear Variables. Tese de Doutorado, Institut für Mathematik, Universität Würzburg, Germany. Citado na pág. 30

Wolfinger(1993) R. Wolfinger. Covariance structure selection in general mixed models. Communications in Statistics - Simulation and Computation, 22(4):1079-1106. Citado na pág. 8,50

Wu(2010) L. Wu. Mixed Effects Models for Complex Data. Chapman and Hall/CRC, Florida. Citado na pág. 25

Zhang e Davidian(2001) D. Zhang e M. Davidian. Linear mixed models with flexible distributions of random effects for longitudinal data. Biometrics, 57(3):795-802. Citado na pág. 1,35

Zhang et al.(2008) P. Zhang, P.X.-K Song, A. Qu e T. Greene. Efficient estimation for patient-specified rates of disease progression using nonnormal linear mixed models. Biometrics, 64(1):29-38. Citado na pág. 35

Zhang e Wei(2008) W. Zhang e H. Wei. Maximum likelihood estimation for simplex distribution nonlinear mixed models via the stochastic approximation algorithm. The Rocky Mountain Journal of Mathematics, 38(5):1863-1875. Citado na pág. 77, 79

Zimprich(2010) D. Zimprich. Modeling change in skewed variables using mixed beta regression models. Research in Human Development, 7(1):9-26. Citado na pág. 18 